Florida International University FIU Digital Commons

3-24-2016

\title{
Maximizing Environmental Sustainability and Public Benefits of Highway Construction Programs
}

Charinee Limsawasd

Florida International University, clims002@fiu.edu

DOI: $10.25148 /$ etd.FIDC000236

Follow this and additional works at: https://digitalcommons.fiu.edu/etd

Part of the Construction Engineering and Management Commons, and the Transportation Engineering Commons

\section{Recommended Citation}

Limsawasd, Charinee, "Maximizing Environmental Sustainability and Public Benefits of Highway Construction Programs" (2016). FIU Electronic Theses and Dissertations. 2488.

https://digitalcommons.fiu.edu/etd/2488 


\section{FLORIDA INTERNATIONAL UNIVERSITY}

Miami, Florida

\section{MAXIMIZING ENVIRONMENTAL SUSTAINABILITY AND PUBLIC BENEFITS \\ OF HIGHWAY CONSTRUCTION PROGRAMS}

A dissertation submitted in partial fulfillment of

the requirements for the degree of

DOCTOR OF PHILOSOPHY

in

CIVIL ENGINEERING

by

Charinee Limsawasd 
To: Interim Dean Ranu Jung

College of Engineering and Computing

This dissertation, written by Charinee Limsawasd, and entitled Maximizing Environmental Sustainability and Public Benefits of Highway Construction Programs, having been approved in respect to style and intellectual content, is referred to you for judgment.

We have read this dissertation and recommend that it be approved.

Arindam Gan Chowdhury

$\begin{array}{r}\hline \text { Irtishad U. Ahmad } \\ \hline \text { Berrin Tansel, Co-Major Professor Laha } \\ \hline \text { Wallied Orabi, Co-Major Professor }\end{array}$

Date of Defense: March 24, 2016

The dissertation of Charinee Limsawasd is approved.

Interim Dean Ranu Jung College of Engineering and Computing

Andrés G. Gil Vice President for Research and Economic Development and Dean of the University Graduate School

Florida International University, 2016 


\section{ACKNOWLEDGMENTS}

First, I would like to express my very great appreciation to my academic advisor Dr. Wallied Orabi for his invaluable advice and constructive suggestions during my research. With his continued support and immense knowledge, it is truly my pleasure to be one of his students and work with him. I would have not been able to receive an achievement in my Ph.D. study without his support and encouragement.

Besides that, I would like to specially thank Dr. Berrin Tansel for her advice and assistance as a committee member and my Co-Major advisor. Her guidance, understanding and invaluable feedback have been very much appreciated. I would also like to extend my grateful thanks to Dr.Arindam G.Chowhury and Dr.Shonali Laha for serving as the members of my dissertation committee and providing insightful comments to improve my research. My grateful thank is also extended to Dr. Irtishad U. Ahmad, Director of the OHL School of Construction, Florida International University, for serving as a committee member and giving me the great opportunity to pursue my Ph.D. study. His understanding and support always have been greatly appreciated.

My special acknowledgement should be given to the OHL School of Construction for the financial support throughout my Ph.D. study. I would also like to thank all faculty, staff, and my colleagues at the OHL School of Construction for a professional, academic, and friendly environment. In addition, I would like to extend my special thanks to Maria Adela Garcia, Carlos Garcia, and Perla Poey for their support, kindness, and warm hearts. With their loves and care, I have always felt surrounding with my family and never feel away from my home. 
Last but certainly not least, I would like to offer my special thanks to Nathee Athigakunagorn for loving, being with me through the good times and bad times, and always staying strong and pulling me up through thick and thin. Finally, the greatest appreciation should be announced to my parents, Weera and Wilaiporn, for their patience, support, and unconditional love. I am gratefully thankful to them for always encouraging and believing in me, and would like to dedicate all of my achievement to them. 


\title{
ABSTRACT OF THE DISSERTATION
}

\section{MAXIMIZING ENVIRONMENTAL SUSTAINABILITY AND PUBLIC BENEFITS \\ OF HIGHWAY CONSTRUCTION PROGRAMS}

\author{
by \\ Charinee Limsawasd \\ Florida International University, 2016 \\ Miami, Florida

\section{Professor Wallied Orabi, Co-Major Professor \\ Professor Berrin Tansel, Co-Major Professor}

Transportation agencies face a challenging task to repair damaged roads in an aging transportation network with limited funding. In addition, the funding gap is forecasted to continue widening, which has direct impacts on the performance of surface transportation networks and the nation's economy in the long run. Recently, transportation agencies were required by a newly enacted law to include national performance-based goals, such as environmental sustainability, in their programming and planning efforts for highway repair and rehabilitation. Therefore, the current practice in the area of highway rehabilitation planning is inadequate to handle this task and new practices are needed to improve the performance of transportation networks while maintain the national goal of maximizing environmental sustainability. Accordingly, this dissertation presents an innovative environmental-based decision-support model for planning highway construction programs. The model is developed in three main parts that are designed to: (1) model total vehicle fuel consumption and public benefits/costs of traveling on transportation networks; (2) evaluate the economic and environmental impacts of highway rehabilitation efforts; and 
(3) develop a multi-objective optimization model to identify and evaluate highway rehabilitation program(s) that are capable of simultaneously minimizing environmental impact and maximizing public benefits of rehabilitation decisions.

First, mathematical models were developed to facilitate estimating the total vehicle fuel consumption and public benefits/cost for road users at the network-level. These models are deigned to estimate vehicle fuel consumption rate, tire depreciation cost, and vehicle repair and maintenance cost rate, in terms of major vehicle-road interaction factors, such as vehicle type, speed, and pavement conditions. The developed and statistically validated models are then used to estimate total vehicle fuel consumption and public benefits/costs at the network-level.

Second, a new model was developed for evaluating the impact of decision making in highway rehabilitation efforts on greenhouse gas emissions and public travel costs. The model has the capabilities of: (1) identifying candidate rehabilitation treatment alternatives for damaged or aging pavement; (2) evaluating the impact of these treatments on pavement performance; (3) estimating network fuel consumption due to highway rehabilitation decisions; (4) estimating additional public costs as a result of travel-delay during road construction operations; and (5) evaluating the impact of rehabilitation efforts on public benefits expressed as expected savings in road user costs.

Third, a multi-objective optimization model was developed to search for and identify highway rehabilitation programs that are capable of minimizing environmental impact in terms of $\mathrm{CO}_{2}$ emissions while maximizing public benefits under budget constraints. This newly developed model enables planners and decision makers to design and implement highway rehabilitation programs that are cost-effective and environmentally-conscious. 
TABLE OF CONTENTS

CHAPTER PAGE

CHAPTER 1

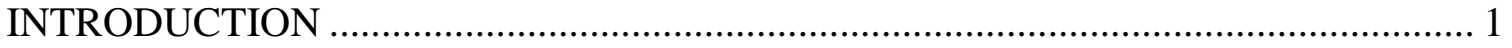

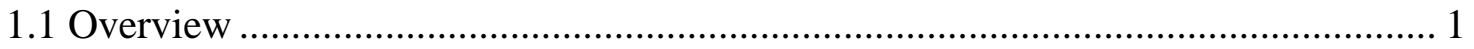

1.2 Problem Statement ......................................................................................... 3

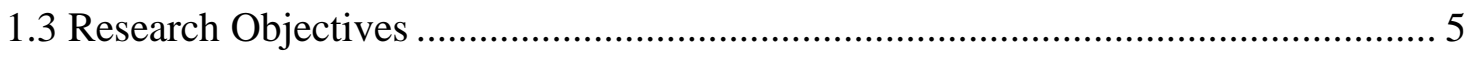

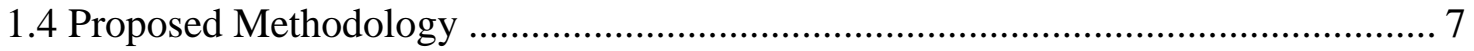

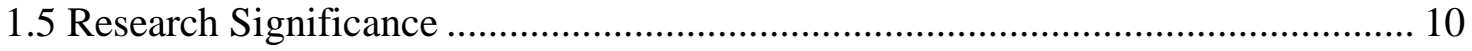

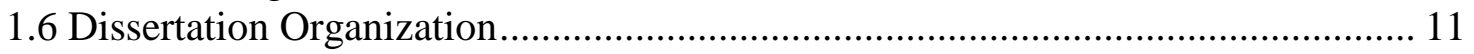

\section{CHAPTER 2}

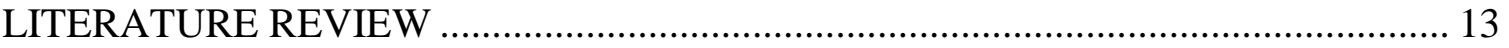

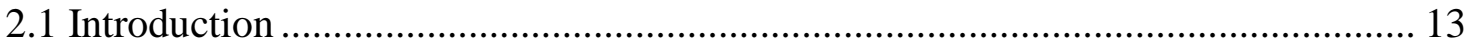

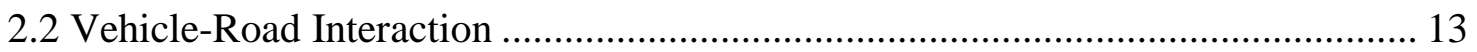

2.2.1 Vehicle Fuel Consumption VS Pavement Roughness.................................... 14

2.2.2 Vehicle Fuel Consumption VS Pavement Surface Type................................. 15

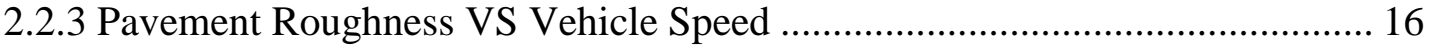

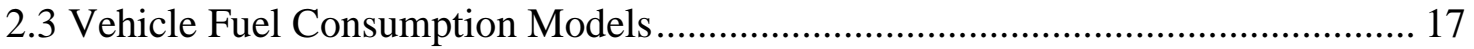

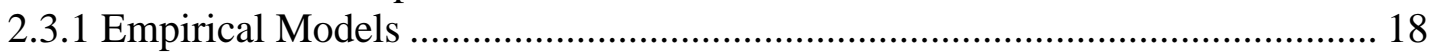

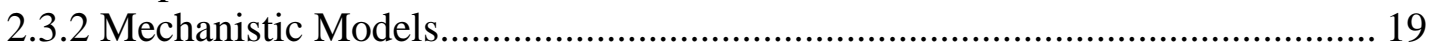

2.4 Highway Construction Programs .................................................................. 20

2.5 Public Benefits and Costs in Highway Transportation ......................................... 22

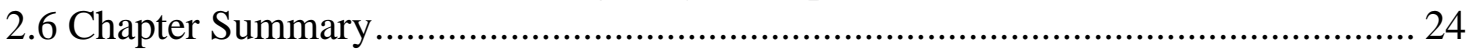

\section{CHAPTER 3}

MEASURING VEHICLE FUEL CONSUMPTION AND PUBLIC BENEFITS IN

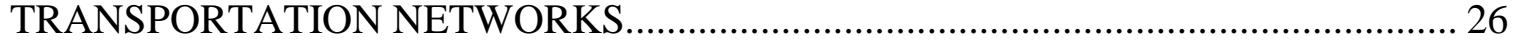

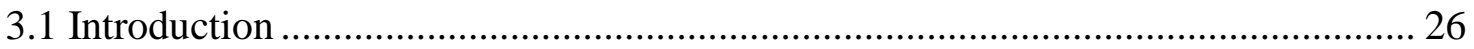

3.2 Development of Mathematical Estimating Modules for Network Application ...... 26

3.3 Vehicle Fuel Consumption Estimating Module ................................................. 28

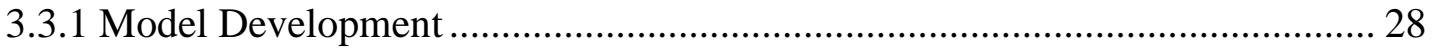

3.3.2 Mathematical Equations for Estimating Fuel Consumption Rate ................... 35

3.4 Tire Depreciation Cost Rate Estimating Module .............................................. 36

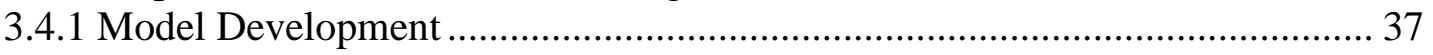

3.4.2 Mathematical Equations for Estimating Tire Depreciation Cost Rate ............. 39

3.5 Repair and Maintenance Costs Rate Estimating Module ..................................... 40

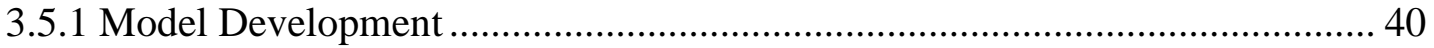

3.5.2 Mathematical Equations for Estimating Repair and Maintenance Costs Rate. 41

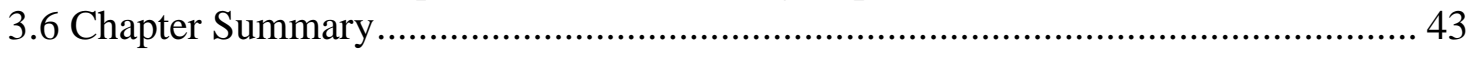




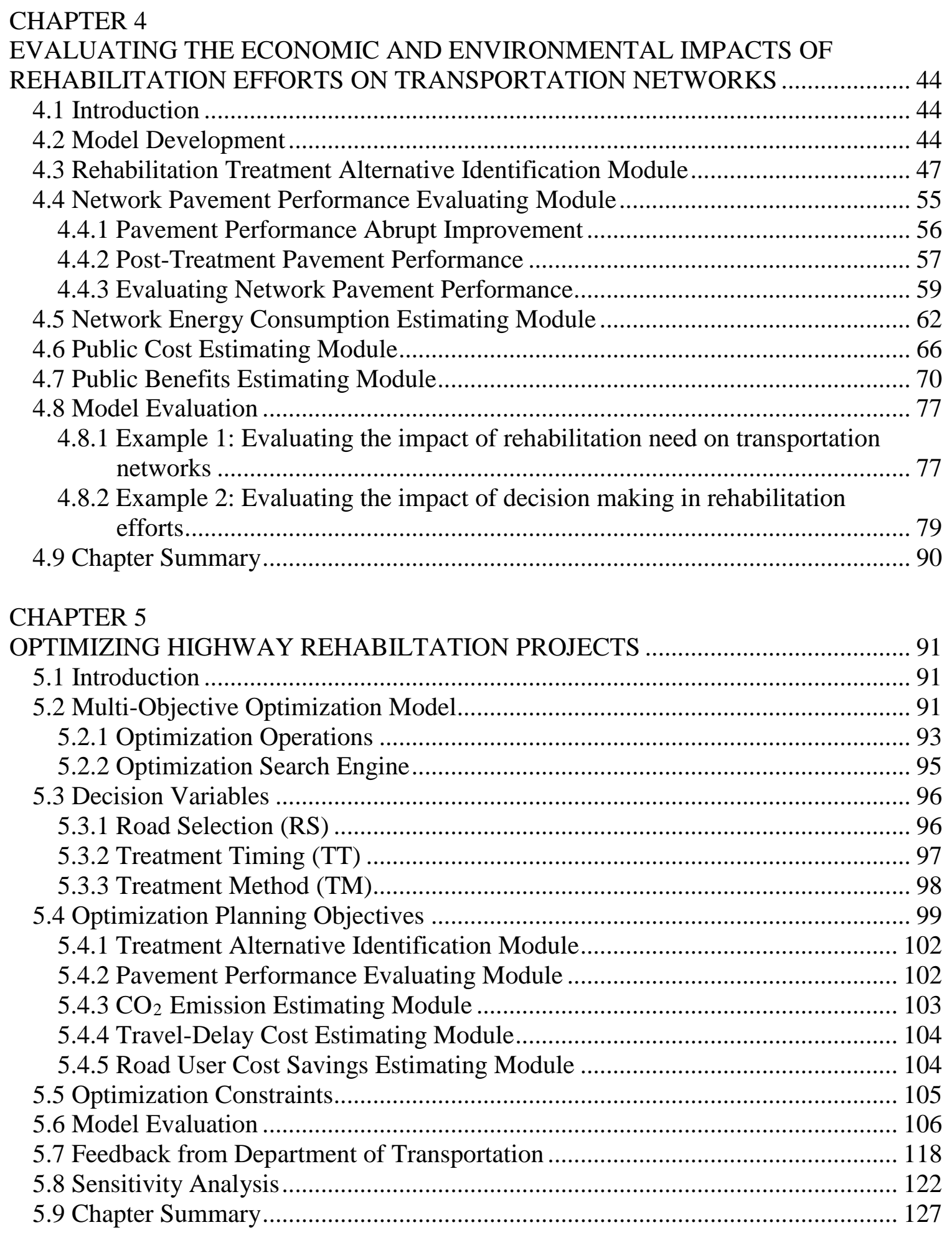


CHAPTER 6

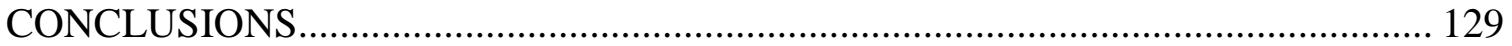

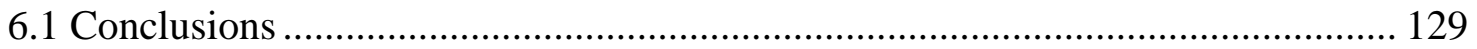

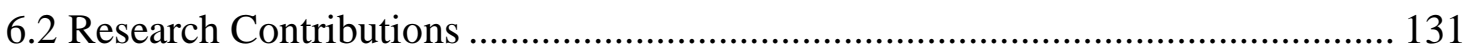

6.3 Limitations and Recommendation for Future Research......................................... 132

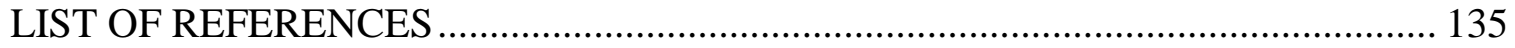

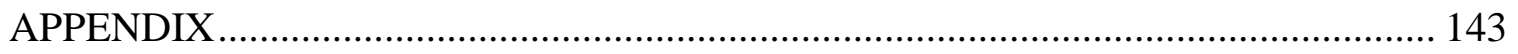

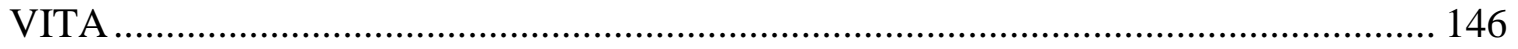




\section{LIST OF TABLES}

TABLE

PAGE

Table 3-1 Vehicle energy consumption estimating equations.....................................36

Table 3-2 Vehicle tire depreciation estimating equations............................................39

Table 3-3 Vehicle repair and maintenance estimating equations.................................42

Table 4-1 Correlations between PCR, IRI, and action category from the past literature..52

Table 4-2 Comparative result in de la Garza and Krueger (2007) .............................52

Table 4-3 Lists of treatment activities from Irfan (2010) for each action category..........53

Table 4-4 Lists of treatment activities from Irfan (2010) for each action category..........54

Table 4-5 Modified parameters for performance jump models.....................................57

Table 4-6 Modified parameters for post-treatment performance models.........................59

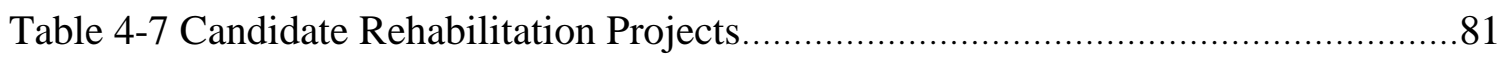

Table 5-1 Candidate rehabilitation projects in Example 1.......................................107

Table 5-2 Average unit cost (in 2015 U.S. dollars/lane-km) for rehabilitation treatment alternatives.

Table 5-3 Average construction duration (day/lane-km) for rehabilitation treatment alternatives. 108

Table 5-4 Candidate rehabilitation projects in Example 2 114 


\section{LIST OF FIGURES}

FIGURE

PAGE

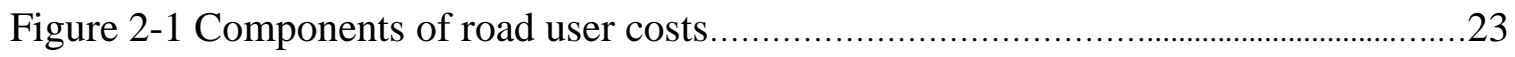

Figure 2-2 Relative vehicle operating costs for trucks .............................................24

Figure 3-1 General flowchart for the equation development .....................................29

Figure 3-2 List of basic parameters in the equation development..................................32

Figure 3-3 Relationships between main factors and fuel consumption rate

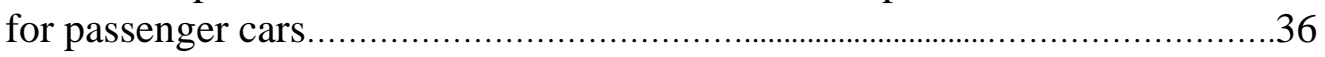

Figure 3-4 Relationships between main factors and tire depreciation rate for passenger cars.

Figure 3-5 Relationships between main factors and repair and maintenance costs

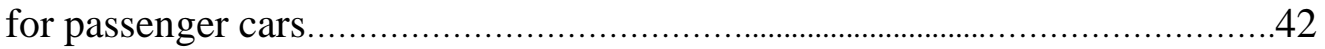

Figure 4-1 Economic and environmental impacts evaluating model.............................47

Figure 4-2 Development of rehabilitation treatment alternative identification module.

Figure 4-3 Modification of possible treatments for pavement condition stages...............54

Figure 4-4 Calculation algorithm for long-term pavement conditions evaluation............60

Figure 4-5 Pavement conditions curve represents the effects of rehabilitation efforts.......62

Figure 4-6 Impact of rehabilitation efforts on energy consumption.............................63

Figure 4-7 Calculation process of transportation network energy consumption...............65

Figure 4-8 Calculation procedure of expected public cost on transportation network.......69 Figure 4-9 Public benefits calculation for transportation network implementation...........74

Figure 4-10 Florida district 4 state highway network ......................................

Figure 4-11 Case study of the damaged transportation network...................................80

Figure 4-12 Impact of rehabilitation treatment selection.......................................83 
Figure 4-13 Total rehabilitation budget needed for each scenario. 85

Figure 4-14 Impact of rehabilitation treatment timing. .87

Figure 4-15 Effect of rehabilitation cost on energy consumption at any year of implementation. .88

Figure 4-16 Impact of length of analysis period on network energy consumption. 89

Figure 5-1 Highway rehabilitation programming and optimization model 92

Figure 5-2 Multi-objective optimization model

Figure 5-3 Population solution for 10 road sections under a 5-year programming horizon. .95

Figure 5-4 Population solution and decision variables under a 20-year horizon plan.

Figure 5-5 Calculation algorithm in evaluating the impact of highway decisions on optimization planning objectives.....

Figure 5-6 Relationship between net public benefits and $\mathrm{CO}_{2}$ emissions per vehicle per lane-mile in Example 1.

Figure 5-7 Overall network pavement conditions over the planning period in Example 1.

Figure 5-8 Comparison of average pavement conditions for each road section.

Figure 5-9 Relationships between rehabilitation cost and (a) public benefits

(b) $\mathrm{CO}_{2}$ emissions in Example

1. 112

Figure 5-10 Comparison of public benefits/cost and $\mathrm{CO}_{2}$ emission savings/cost between two groups of rehabilitation programs in Example 1.

Figure 5-11 Relationship between net public benefits and $\mathrm{CO}_{2}$ emissions per vehicle per lane-mile in Example 2.

Figure 5-12 Overall network pavement conditions over the planning period in Example 2.

Figure 5-13 Relationships between rehabilitation cost and (a) public benefits

(b) $\mathrm{CO}_{2}$ emissions in Example

2 117 
Figure 5-14 Comparison of public benefits/cost and $\mathrm{CO}_{2}$ emission savings/cost between two groups of rehabilitation programs in Example 2

Figure 5-15 Sensitivity analysis from an uncertainty in initial pavement conditions ......122

Figure 5-16 Sensitivity analysis from an uncertainty in budget availability.

Figure 5-17 Sensitivity analysis from an uncertainty in Benkelman Beam rebound deflection.

Figure 5-18 Sensitivity analysis from an uncertainty in vehicle acceleration....................125

Figure 5-19 Sensitivity analysis from an uncertainty in mean profile depth...................126

Figure 5-20 Sensitivity analysis from an uncertainty in gradient....................................126 


\section{CHAPTER 1}

\section{INTRODUCTION}

\subsection{Overview}

Transportation planning agencies face a challenging task to repair and upgrade the nation's poor and congested roadway network under a steep funding gap (Dhakal and Oh 2011; Zhang et al. 2012). The American Society of Civil Engineers (ASCE) forecasts that the continuation of the status quo can have dire impacts on travelers and the economy in the long run (ASCE 2013). Current highway program planning efforts use need-based adhoc methods to allocate the limited funding to competing projects. This leaves much room for improvement to include important factors that maximizes the public benefit from surface transportation (Sathaye and Madanat 2011; Sharaf and Mandeel 1998). The Moving Ahead for Progress in the 21st Century Act (MAP-21) of 2012 is an example of the Federal Government's plans to shift transportation planning efforts towards performance-based methods by allocating available budget to projects that serve specific national goals (FHWA 2012). This places more pressure on transportation planning agencies in order to incorporate goals such as safety, environmental sustainability, and system reliability into their planning and programming efforts.

Considering environmental sustainability, transportation is responsible for $28 \%$ of greenhouse gas (GHG) emissions (EPA 2013b). This number primarily results from the fossil fuel combusted in motor vehicles (EPA 2013a), which accounts for 636 billion liters (168 billion gallons) of fuel every year (FHWA 2014). Over 90\% of fuel used in transportation, which includes gasoline and diesel, is based on the petroleum refining process (Kahn Ribeiro et al. 2007). This combustion of fossil fuels can have drastic impact 
to the environment by increasing the amount of $\mathrm{CO}_{2}$ emitted to the atmosphere and causing climate change. To this end, controlling and reducing fuel used in surface transportation as a result of an improved highway rehabilitation decision-making process can support transportation agencies in setting and implementing new policies to reduce $\mathrm{CO}_{2}$ emissions in transportation networks. Therefore, minimizing environmental impact in terms of $\mathrm{CO}_{2}$ emissions can have a significant impact on achieving the national goal of improving sustainability in transportation networks.

Accordingly, highway construction programs should consider the impact of highway maintenance and rehabilitation projects on environment. Furthermore, these planning efforts should include searching for and implementing maintenance and rehabilitation plans that are capable of minimizing environmental impact among other planning objectives. To this end, reducing energy consumption in transportation should be adopted in the decision making process by controlling some myriad factors including traffic volume, vehicle type, vehicle speed, and pavement conditions (Chatti and Zaabar 2012). For example, several research studies emphasized on the significant impact of pavement conditions on fuel consumption (Amos 2006; Wang et al. 2012; Zaabar and Chatti 2010) and therefore GHG emissions (Lidicker et al. 2012; Zhang et al. 2010). Therefore, the selection of which roads to resurface or widen can significantly reduce/increase the environmental impact generated from fuel consumed by vehicles travelling on that road.

In addition, highway construction programs have a direct and significant impact on public benefits and/or costs. Decisions made for these construction programs affect the planning objectives of reducing congestion, increasing travel safety, minimizing travel 
time, decreasing road user costs, and stimulating local economy among other objectives. These decisions need to be made subject to limited and insufficient budgets. It is therefore important to optimize decision-making in highway construction problems in order to reduce environmental impact and maximize public benefits.

Accordingly, there is a pressing need for new research in the area of decisionmaking for highway maintenance and rehabilitation efforts in order to improve the environmental sustainability of surface transportation networks by reducing $\mathrm{CO}_{2}$ emissions while maximizing public benefits from the road repair and upgrade works.

\subsection{Problem Statement}

Optimizing highway construction programs to minimize environmental impact and maximize public benefits is a challenging and complex task. This is mainly due to the myriad factors and their relationships that need to be analyzed and modeled at different levels of analyses. First, the impact of the factors related to vehicle-road interaction need to be analyzed and modeled in such a way that facilitate further analysis at the networklevel to estimate total energy consumption, and therefore $\mathrm{CO}_{2}$ emissions in transportation networks. Second, total energy consumption and expected public benefits/costs of highway maintenance and rehabilitation work need to be analyzed and modeled. Third, highway construction programs should be optimized in order to minimize environmental impact in terms of $\mathrm{CO}_{2}$ emissions and maximize public benefits, simultaneously.

Majority of GHG emissions is carbon dioxide that enters to atmosphere through burning fossil fuel, which is the main source of energy consumption in transportation (EPA 2013a; EPA 2013b). It is therefore important to analyze and model vehicle fuel 
consumption in order to facilitate evaluation of total energy consumption of highway construction programs. Vehicle fuel consumption is a direct result of vehicle-road interaction. Therefore, factors such as vehicle type and size, travel speed, traffic volume, and road conditions are important factors in evaluating vehicle fuel consumption. These factors and their relationships must be analyzed and modeled. Existing research focused on: (i) analyzing the relationship between fuel consumption and pavement roughness (Amos 2006; Epps et al. 1999; Watanatada et al. 1987); (ii) estimating fuel consumption as a factor of road conditions only without regard to important factors such as vehicle type and travel speed (Yu and Lu 2012; Zhang et al. 2009); and (iii) estimating fuel consumption based on detailed and very specific data unsuitable for upper level analyses (Bennett and Greenwood 2003; Chatti and Zaabar 2012; Zaabar and Chatti 2010). Despite of the significant contributions of these studies, there is no reported research that provided a method to estimate vehicle fuel consumption considering all relevant vehicle-road factors using data readily available to planners and decision makers.

The analysis and evaluation of total energy consumption and expected public benefits of highway construction programs are keys to solving this problem. Vehicle fuel consumption must be aggregated at the network level to account for traffic assignment changes due to repair and upgrade works. In addition, public benefits from savings in travel time and road user costs should also be analyzed and evaluated. Furthermore, the impact of deterioration in road conditions over time should be analyzed to provide more accurate evaluation of repair and upgrade decisions on environmental impact and public benefits.

There is typically a wide range of alternatives in decision-making for highway construction programs. Each of these alternatives has a different impact on environment 
and public benefits. It is therefore important for planners and decision makers to be able to search for and identify the construction program(s) that can minimize environmental impact and maximize public benefits, simultaneously. This is a multi-objective and constrained optimization problem that should be modeled based on the factors mentioned above. The decision variables and planning objectives of this optimization problem should be modeled in an effective and efficient manner. In addition, the optimization objectives are nonlinear and non-continuous, which require the selection of a suitable multi-objective optimization technique capable of handling such problems.

\subsection{Research Objectives}

The main goal of this study is to develop an environmentally-conscious decisionsupport model for planning highway construction programs. Three research objectives are identified to achieve this main goal, along with the research questions and hypotheses, which can be described as follows:

\section{(1.3.1) Objective 1}

Develop a vehicle fuel consumption estimating module that takes into consideration the main vehicle-pavement interaction factors and can facilitate estimating total fuel consumption at the network-level.

Research Questions:

(1.3.1.1) What are the main variables that affect vehicle fuel consumption?

(1.3.1.2) How do the main variables affect fuel consumption?

(1.3.1.3) How can these variables be modeled to estimate fuel consumption? 


\section{Hypothesis:}

Vehicle type, vehicle speed, and pavement conditions have a significant impact on fuel consumption, and must be integrated in decision making for highway construction programs.

\section{(1.3.2) Objective 2}

Develop a model to estimate the impact of decision making in highway construction programs on total energy consumption and public benefits/costs under budget constraints.

Research Questions:

(1.3.2.1) What are the main factors of highway construction programs that should be considered for evaluation?

(1.3.2.2) How can vehicle fuel consumption be aggregated to estimate total energy consumption of a specific highway construction program?

(1.3.2.3) How public benefits/costs of highway construction programs are modeled and evaluated?

Hypothesis:

Highway construction programs have a significant impact on total energy consumption and potential public benefits/costs such as savings in road user costs.

\section{(1.3.3) Objective 3}

Develop a multi-objective optimization model to search for and identify highway construction program(s) that are capable of simultaneously minimizing environmental impact and maximizing public benefits subject to budget constraints. 


\section{Research Questions:}

(1.3.3.1) What are the main decision variables that should be modeled and optimized to minimize environmental impact and maximize public benefits?

(1.3.3.2) How can the impacts of the decision variables and constraints on the optimization objectives be modeled and analyzed?

(1.3.3.3) Which optimization technique is best suited to search for and find optimal solution(s) to this problem?

Hypothesis:

Decision making in highway construction programs can be optimized to find optimal program(s) that can minimize environmental impact and maximize public benefits under budget constraints.

\subsection{Proposed Methodology}

The research methodology is classified into four main tasks to support the aforementioned objectives as: (1) establish the knowledge base of vehicle fuel consumption in highway transportation by executing a comprehensive literature review, and observing research gaps in the current body of knowledge, (2) develop the vehicle fuel consumption estimating module for highway transportation, (3) develop a model for estimating total energy consumption and public benefits in the network, and (4) develop a multi-objective optimization model for highway construction programs. The details of each task can be explained as follows: 
(1.4.1) Conduct a comprehensive literature review and identify the research gaps

In this task, the relevant literature review is comprehensively examined to establish the knowledge base, and specify the research gaps in the area. The four following activities can be identified to fulfill this task:

(1.4.1.1) Investigate previous research that concentrates on vehicle-road interaction that has a substantial impact on fuel consumption.

(1.4.1.2) Review existing vehicle fuel consumption models.

(1.4.1.3) Explore research studies regarding highway construction programs.

(1.4.1.4) Examine research works regarding public benefits/costs in highway transportation.

\section{(1.4.2) Develop vehicle fuel consumption estimating module}

The objective of this task is to model the vehicle fuel consumption estimating module for highway transportation that takes into consideration pavement conditions and vehicle speeds. This step can be classified into four subtasks, as shown in the details below:

(1.4.2.1) Investigate the main factors affecting vehicle fuel consumption.

(1.4.2.2) Investigate the relationships between vehicle type, vehicle speed, and pavement conditions to vehicle fuel consumption.

(1.4.2.3) Develop the model for estimating vehicle fuel consumption in highway transportation.

(1.4.2.4) Evaluate and refine the model by verifying with the data from the field investigation. 
(1.4.3) Develop a model to estimate total network energy consumption and public benefits

The objective of this task is to develop the model for estimating the impact of decision making in highway construction programs on energy consumption and public benefits/costs under budget limitation. The following steps can fulfill this objective as:

(1.4.3.1) Investigate the significant factors in highway construction programs affecting energy consumption and public benefits/costs.

(1.4.3.2) Integrate the fuel consumption module to highway construction programs.

(1.4.3.3) Design and implement the fuel consumption module in estimating public benefits/costs.

(1.4.4) Develop a multi-objective optimization model for highway construction programs

The main objective of this task is to develop the optimization model for highway construction programs, by simultaneously minimizing environmental impact and maximizing public benefits within budget constraints. The following activities are performed to accomplish the main objective:

(1.4.4.1) Identify the main decision variables for minimizing environmental impact and maximizing public benefits.

(1.4.4.2) Evaluate the impacts of decision variables and constraints on the optimization objectives.

(1.4.4.3) Develop the optimization model to find optimal highway construction programs under budget constraints. 


\subsection{Research Significance}

This research study is devised to promote the environmental sustainability in decision making of highway rehabilitation planning by developing a robust optimization model that is able to effectively facilitate the rehabilitation investment of transportation agencies. The result of this research provides significant contributions on society and transportation agencies as follows.

\section{Contribution on Society}

This research study significantly benefits to society in terms of both economy and environment. The effective highway rehabilitation plans can promote the sustainability commitment to mitigate the environmental impact especially greenhouse gas (GHG) emissions from a large amount of energy consumed in vehicle operating on transportation networks. An effective rehabilitation implementation can also increase the public benefits, such as the savings in road user costs and the reduction in traffic delay, on transportation networks. Moreover, optimizing an allocation of the limited rehabilitation funding under the model developed in this study can maintain the performance-based goals of transportation agencies for environment and public benefits simultaneously.

\section{$\underline{\text { Contribution on Transportation Agencies }}$}

This research also advances and enhances decision making in highway rehabilitation efforts. The model developed in this study can increase the level of sustainability in highway rehabilitation by spending the public financial resources in a more effective and beneficial manner. This paradigm shift also encourages state departments of transportation to supports the Federal's performance goals in highway 
transportation by taking environmental sustainability into their planning and programming efforts.

\subsection{Dissertation Organization}

This dissertation consists of six main chapters that are relative to the main objectives of this study. The organization of this dissertation are consecutively presented as follows.

Chapter 1 (Introduction) provides the overall background and motivation of this study. This chapter contains the research overview, problem statement, research objectives, research methodology, and the contribution of the research to the society and transportation entity.

Chapter 2 (Literature Review) provides a comprehensive review of all relevant studies that concentrate on vehicle-road, review existing vehicle fuel consumption models, explore past studies on highway construction programs, and examine research works about public benefits and public costs in highway transportation. This review also provides the research gaps and underline the significance of this research study.

Chapter 3 (Measuring Vehicle Fuel Consumption and Public Benefits on Transportation Networks) presents the development of mathematical models that is capable of facilitating the transportation network analysis. The models in this chapter are statistically developed to contributing the estimation of vehicle fuel consumption rate, tire depreciation cost rate, and repair and maintenance costs rate. The results calculated from the mathematical equations in this chapter will be used as input parameters for evaluating the impact of highway rehabilitation efforts on the entire transportation network, which will be subsequently formulated in the chapter 4 . 
Chapter 4 (Evaluating the Economic and Environmental Impacts of Rehabilitation Efforts on Transportation Networks) presents a novel model to support an evaluation of impacts resulting from highway rehabilitation efforts on transportation networks. The developed model is capable of: (1) assigning a rehabilitation treatment to the selected deteriorating pavement; (2) forecasting the long-term pavement conditions resulting from the implementation of highway rehabilitation efforts; (3) evaluating the impact of rehabilitation efforts on total network energy consumption; (4) measuring the impact of network rehabilitation implementation on public cost; and (5) evaluating total public benefits resulting from the rehabilitation implemented throughout transportation networks.

Chapter 5 (Optimizing Highway Rehabilitation Efforts) presents the development and application of a new model for optimizing highway rehabilitation programs that is capable of providing optimal tradeoffs between minimizing environmental impact and maximizing net public benefits. This chapter introduces the framework of the developed multi-objective optimization model. The descriptions of decision variables, planning objectives, and optimization constraints are provided afterwards. At the end, the case study of the transportation network is also analyzed to demonstrate the capabilities of the developed model.

Chapter 6 (Conclusions) provides a summary and conclusions of this dissertation. It also presents the contributions of this research study, limitations and recommendations for future works. 


\section{CHAPTER 2}

\section{LITERATURE REVIEW}

\subsection{Introduction}

This part presents the literature review of existing research studies that are associated with the estimation of vehicle fuel consumption in transportation. The literature review can be divided into four parts: (1) investigate research studies that concentrate on the variability of fuel consumption due to pavement characteristics and vehicle-related factors, or vehicle-road interaction, (2) review existing vehicle fuel consumption models, (3) explore previous research regarding highway construction programs, and (4) examine research works regarding public benefits/costs in highway transportation.

\subsection{Vehicle-Road Interaction}

This section compiles existing research about vehicle-road interaction, mainly with respect to vehicle fuel consumption influenced by pavement characteristics. Two main components have been widely mentioned in this research area - pavement roughness, and pavement surface type. In fact, both of them are highly correlative, as the different types of surface could have some effects on pavement roughness. Besides the roughness and surface type, research corresponding to vehicle speed due to the roughness variability is mentioned here, as vehicle speed is asserted in some studies as another parameter that affects vehicle fuel consumption. 


\subsubsection{Vehicle Fuel Consumption VS Pavement Roughness}

Many studies have concentrated on the impact of pavement performance on vehicle fuel consumption (Zaabar and Chatti 2011). Pavement roughness is one commonly used index for measuring pavement performance. Roughness level is typically addressed by the international roughness index (IRI) that was developed by the World Bank (Sayers et al. 1986). The IRI adopts the concept of displacement measurement in pavement along the vehicle's travel distance, and it can be represented in meter per kilometer or inch per mile (Lidicker et al. 2012; Zhang et al. 2009). The IRI value can range from zero for perfectly smooth pavement and has no upper bound (Zhang et al. 2009), with a typical value between 1 to $5 \mathrm{~m} / \mathrm{km}$ (Wang 2013).

Pertaining to the impact of roughness on fuel consumption, most studies have investigated this effect with specific scopes, for example, focusing on specific types of vehicles or performing at a constant vehicle speed. Amos (2006) studied the effect of road smoothness based on real conditions, by constructing the experiment in Missouri. Four dump trucks were driven at $96.6 \mathrm{~km} / \mathrm{h}(60 \mathrm{mph})$ on the road before and after the pavement resurfacing. The result of fuel economy was calculated, and it was found that the smoothness of pavement surface has an impact on vehicle fuel consumption. Numerically, $53 \%$ improvement in the smoothness can contribute to approximately $2.5 \%$ of fuel saving.

The impact of pavement roughness was also observed in the WestTrack project, which was supported by the Federal Highway Administration (FHWA) and the National Cooperative Highway Research Program (NCHRP) (Epps et al. 1999). The test was performed by using two trucks driving at a speed of $64.4 \mathrm{~km} / \mathrm{h}$ (40 mph) on two different pavements of roughness. The result shows the effect of fuel consumption due to the 
difference in pavement roughness. The larger consumption rate is indicated from 1.79-1.87 $\mathrm{km} / \mathrm{liter}$ when the tested vehicles traveled on the $1.18-\mathrm{m} / \mathrm{km}$ IRI, compared to the pavement with the IRI of $2.37 \mathrm{~m} / \mathrm{km}$.

Some research studies establish arithmetical models to describe the correlation between fuel consumption and the different roughness, which can be found in Zhang et al. (2009), and Yu and Lu (2012). Both studies introduce the correlation in terms of the fuel consumption factor (FCF) that represents the linear proportion of fuel consumption on the observing pavement and on an ideally smooth pavement surface. Specifically, the first paper restrictedly proposes the equation for heavy-duty trucks with FCF $=0.0397 \mathrm{IRI}+$ 0.9524 (Zhang et al. 2009). The second represents the correlations for passenger cars and trucks in the simple format as: $\mathrm{FCF}=0.007377 \mathrm{IRI}+0.993$ for passenger cars, and FCF $=$ 0.02163 IRI +0.953 for trucks (Yu and Lu 2012).

Based on literature review, most studies reach the similar conclusion that the pavement roughness, IRI mentioned herein, has a significant impact on the fuel consumption rate of the vehicle traveling on the road. However, those studies have their main objective to specifically investigate the effect from the roughness change, and do not aim to inclusively examine all parameters for accuracy. As a consequence, they limit their scopes by excluding some potential variables (e.g. vehicle speed) in their considerations.

\subsubsection{Vehicle Fuel Consumption VS Pavement Surface Type}

There is a significant amount of research that investigates the effect of pavement type to fuel consumption. For non-truck vehicles, the findings seem to be identical with most of the associated studies that conclude that there is no significant difference in fuel consumption between flexible and rigid pavements (Sumitsawan et al. 2009; Taylor and 
Patten 2006; Zaabar and Chatti 2010; Zaniewski 1989; Zaniewski et al. 1982). Compared to non-truck vehicles, trucks are reported to save on concrete pavement in comparison with the asphalt surface, except in Zaniewski et al. (1982), which claimed no variation. This inconsistency can be from the age of the study, in which modern technology was not applied to truck when the Zaniewski et al. (1982) was performed. The examples of studies that declare difference in fuel consumption due to different pavement surfaces are Taylor et al. (2002), Taylor and Patten (2006), and Zaabar and Chatti (2010).

However, due to the discrepancy in the amount of consumption savings mentioned, Zaabar and Chatti (2011) performed the in-depth examination for the passenger car, van, SUV, and truck, particularly in different weather conditions. Based on their results, fuel consumptions among flexible and rigid surfaces for the passenger car, van, and SUV are not statistically significant, for both summer and winter conditions. Nevertheless, it shows that fuel consumption in trucks is significantly different, at approximately $4 \%$, only in the summer at a low operating speed. The other conditions, driving at the high speed in the summer, and driving in the winter, do not statistically reveal a significant difference. Conclusively, the limited effect occurs when the truck is traveling on the asphalt surface with a low speed in the hot weather.

\subsubsection{Pavement Roughness VS Vehicle Speed}

Due to the importance of speed to vehicle fuel consumption, some studies are stated here to clarify the effect of pavement roughness to vehicle speed. As a matter of fact, the current research refers to a limited impact on vehicle operating speed in relation to surface smoothness. Zaniewski et al. (1982) denoted the linear speed reduction based on changes in pavement roughness. They tested the impact corresponding to the range of speed from 
24.1 to $56.3 \mathrm{~km} / \mathrm{h}$, by assuming the baseline at $24.1 \mathrm{~km} / \mathrm{h}$ with no impact. The impact is basically represented as mathematical equations and it is utilized for generating the speed adjustment factor in the simple and absolute form. The result confirms the very limited impact, especially when the IRI reduction is less than $3 \mathrm{~m} / \mathrm{km}$.

Another study was performed by Wang (2013), in which the influence of pavement roughness to the free-flow speed is investigated. This study mainly intends to test changes in driving behavior, which directly coincide with vehicle speed, with respect to surface roughness. The findings represent the linear regression model that verifies a very restricted correlation between pavement roughness and vehicle speed. Quantitatively, $0.48-0.64$ $\mathrm{km} / \mathrm{h}$ in speed can be decreased as an increase in $1 \mathrm{~m} / \mathrm{km}$ of IRI value. This number substantially confirms the limited impact of speed change due to the roughness. Concerning these two existing studies, they relatively agree on the conclusion that expresses the limitation of vehicle speed change corresponding to the roughness alteration.

\subsection{Vehicle Fuel Consumption Models}

Existing vehicle fuel consumption models can be categorized into two main groups, as the empirical and mechanistic models (Chatti and Zaabar 2012). The first is generally based on the observation or field experiment, in which the relationship is unspecifiable in mathematical format. It is usually scoped to apply only in specific variables. Contrastingly, the mechanistic model is developed by taking into consideration the mechanical theory to allow the improvement in the first model type. It additionally takes into account the factors related to engine attributes and the mechanical power in the analysis. The examples of fuel consumption models in each category, including their explanations, are briefly provided as follows. 


\subsubsection{Empirical Models}

The starting point of the vehicle fuel consumption study in the United States dates back to year 1969, when Winfrey initiated information for estimating the fuel consumption cost, by depending on types of vehicles (Chatti and Zaabar 2012; Zaabar and Chatti 2010). Further investigation was executed by taking into account pavement conditions in Zaniewski et al. (1982). However, the test was performed by measuring fuel consumption in different pavement conditions at the constant speed. Many important factors were excluded from the experiment, for example, the acceleration rate and idling. Its result utterly contradicts the findings found in the later studies. Pavement conditions show no significant influence to fuel consumption in this study, while the others afterwards display the strong relationship. This can be explained by many deficiencies in Zaniewski et al. (1982), for instance, the limitation of sample size and technology differences.

Subsequently, there are many studies proposing the relationship in the form of mathematical models. For example, the models that are exemplified and aforementioned in the vehicle-road interaction section (e.g. Zhang et al. (2009), and Yu and Lu (2012)) include only the effect from pavement roughness. In addition, there is an attempt to directly measure fuel consumption from the vehicles’ performance, such as engine torque and gear level, instead of pavement roughness. Klaubert (2001) empirically incorporated the mechanical power in his model, and developed the regression equation for the fuel consumption estimation. 


\subsubsection{Mechanistic Models}

This type of model integrates mechanical principles for model construction. It encompasses actual forces generated in the vehicles' engine, for example aerodynamic forces, rolling resistance forces, and inertial forces. It is worth noting that most of the models have extended from the prior developed models in some dimensions (Chatti and Zaabar 2012). The major models are the South African model, the Australian model (ARFCOM), and the World Bank HDM-4 model, which is the subsequent version of the HDM-3 model (Chatti and Zaabar 2012). The details of models are given as follows.

First, the South African model was established by taking the tractive force requirement, vehicle speed, and the fuel efficiency factor into consideration (Chatti and Zaabar 2012). However, this model was improved later by including the impact of vehicle acceleration on the fuel efficiency factor (Chatti and Zaabar 2012). Therefore, both effects in the steady and acceleration states are placed in the new model.

Subsequent research reveals that the fuel efficiency is influenced by engine power, in addition to tractive forces. The ARFCOM model was eventually constructed to include engine and accessories power in the analysis (Chatti and Zaabar 2012). This model proves to fulfill the practicality and soundness criteria, in which it considers both the impacts from pavement conditions, and emerging technologies (Chatti and Zaabar 2012). However, the most popular model for estimating fuel consumption is the HDM-4 model that was launched by the World Bank (Bennett and Greenwood 2003). This latest model adopts the previous ARFCOM model to comprehensively update the calculation by including more potential factors, regarding engine speed, accessories power, and engine drag (Chatti and Zaabar 2012; Zaabar and Chatti 2010). This updated model is currently implemented in 
many countries around the world, and there is an effort to calibrate it to be applicable to the U.S. conditions in Chatti and Zaabar (2012).

\subsection{Highway Construction Programs}

There have been many research works that adopted optimization techniques to effectively plan the infrastructure system. Highway is one system that has been gaining interests, in which most concerns are usually focused on how to manage the system within the resource limitation and receive effective outcomes. Due to the stringent budget, highway agencies have rigorously confronted the difficulties in fund allocation to roads in the network, and obtain the most beneficial utility. Many notable works are mentioned herein to depict the concept of the optimization in the network-level planning.

The implementation of an optimization approach can be initially acknowledged to Chan et al. (1994), in which they represented the capability of genetic algorithm (GA) for road maintenance planning. In this paper, the theory and operational mechanism of GA are described and attached to the simple application in the road-network system. The case scenario is hypothetically established with the objectives to minimize the present worth of maintenance costs within budget constraints. Subsequently, the optimization has been applied in network planning in the different points of view. Wang and Liu (1997) found the optimal program for the network pavement system by allocating limited financial resources to get the maximization of overall network performance.

Fwa et al. (2000) developed the multi-objective GA optimization model for the maintenance plan at the network level. They aimed to (1) maximize the work production, (2) minimize the maintenance cost, and (3) maximize the pavement network's performance simultaneously under the conditions of production requirements, manpower and equipment 
availabilities, time constraints, and budget constraints. Ferreira et al. (2002) additionally adopted a probabilistic approach for evaluating pavement conditions, and incorporated it in the network-level optimization. The programming is performed with the effort to minimize maintenance and rehabilitation costs, and contemporarily accomplished the minimum requirement of pavement performance. With the similar concept, the optimization program was developed for the rural pavement network in Kerala, India (Mathew and Isaac 2014). The researchers deterministically developed the optimal scheme with the maintenance cost minimization and pavement performance maximization, subject to budget limitations and the minimum requirement of network-pavement conditions.

Regarding the aforementioned studies, maintenance cost is considered by only agency cost, which is the cost for maintaining and repairing facilities. However, there are some works that deliberately take into account road user costs, which involve cost components from users traveling on roads (e.g. travel time, and vehicle operating cost). Orabi and El-Rayes (2011) adopted the GA optimization approach to allocate the limited budget, under maximizing benefits and minimizing network service disruption. A savings in road user costs is taken into consideration for the benefit evaluation. In addition, Sathaye and Madanat (2011) proposed the basic optimization model for programming the networkpavement system within the limited budget. The research team allows both agency construction costs and road user costs for the analysis. This developed model was expanded later with application in the large-scale networks in Sathaye and Madanat (2012).

The optimization technique is also employed in an executive perspective. For instance, it was implemented in allocating highway funds among multi-regional public organizations at the administrative level (Chan et al. 2003), to certain requirements of each 
authority. Another example is Fwa and Farhan (2012), who primarily applied the optimization to each single individual asset system, and successively optimized the budget allocation strategy across the infrastructure system.

Recently, optimizing in the network system is extended by integrating the sustainability principle. In Lidicker et al. (2012), the model was developed to minimize agency and user costs, while minimizing environmental impacts, in terms of GHG emissions. The trade-off relationship between maintenance costs and emissions is proposed, although the study is concentrated at the project level. Another associated work embraces the concept of life-cycle costs (Zhang et al. 2012). The energy consumption, GHG emissions, and maintenance costs are integrated into one single objective to determine the optimal plan under the restriction of allocated budget and overall pavement performance.

\subsection{Public Benefits and Costs in Highway Transportation}

Highway transportation costs can be categorized into agency costs and road user costs (Zaniewski 1989). The first is basically associated with highway agency costs for constructing or maintaining facilities, while the other is due to users traveling on roads that accounts for costs related to travel time and vehicle operation. Compared to construction or maintenance costs, road user costs seem to be relatively larger (Zaabar and Chatti 2011), as vehicle fuel consumption plays one of the major roles in highway transportation (Dewan and Smith 2002). Therefore, reducing road user costs, such as reducing travel time, and vehicle fuel consumption, can significantly contribute to saving a great amount of money in the transportation system. 
In reference to public benefits, a savings in road user costs can be addressed as benefits from implementing highway construction programs (Sharaf and Mandeel 1998). With specific maintenance and rehabilitation methods applied to road sections, the pavement conditions are expected to upgrade. Therefore, benefits can be measured in terms of cost reduction and a decrease of environmental impacts, due to the application of different maintenance and rehabilitation alternatives (Sharaf and Mandeel 1998).

Connections among the fuel consumption cost, vehicle operating costs, and road user costs have mutually substantial relationships. In fact, road user costs are all cost components caused by road users traveling on roads. Road users attain a variety of impacts, consisting of travel time costs, accident costs, environmental impacts, and vehicle operating costs, safety, and convenience (Bennett and Greenwood 2003; Chatti and Zaabar 2012). The following figure, Figure 2-1, is modified from associated references to describe road user costs.

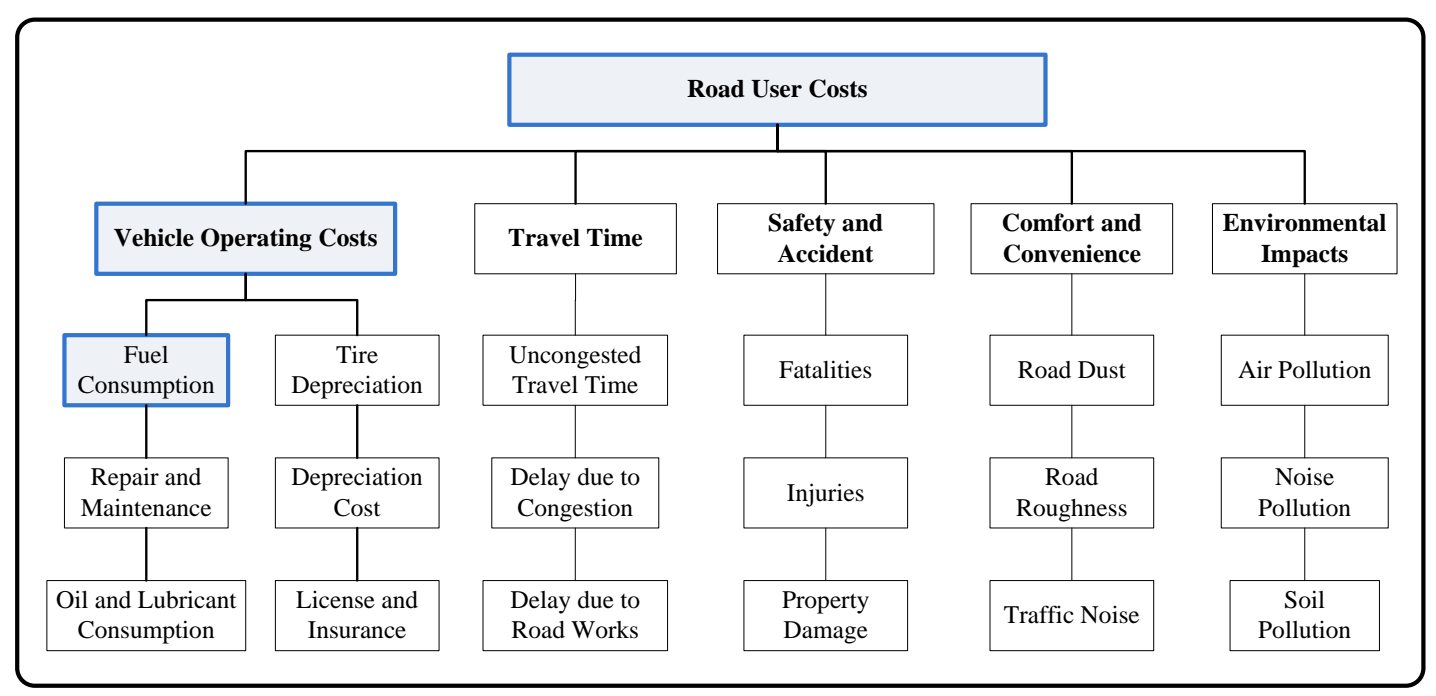

Figure 2-1 Components of road user costs (Bennett and Greenwood 2003; Chatti and Zaabar 2012) 
Fuel consumption cost is the primary element of vehicle operating costs. The other components, which are tire wear cost, maintenance and repair cost, depreciation cost, as well as license and insurance, could be counted as secondary (Chatti and Zaabar 2012). Statistically, the fuel consumption cost addresses the largest portion in total vehicle operating costs (Barnes and Langworthy 2004). The quantitative values are retrieved and graphically presented under the modification of Chatti and Zaabar (2012), as illustrated in Figure 2-2.

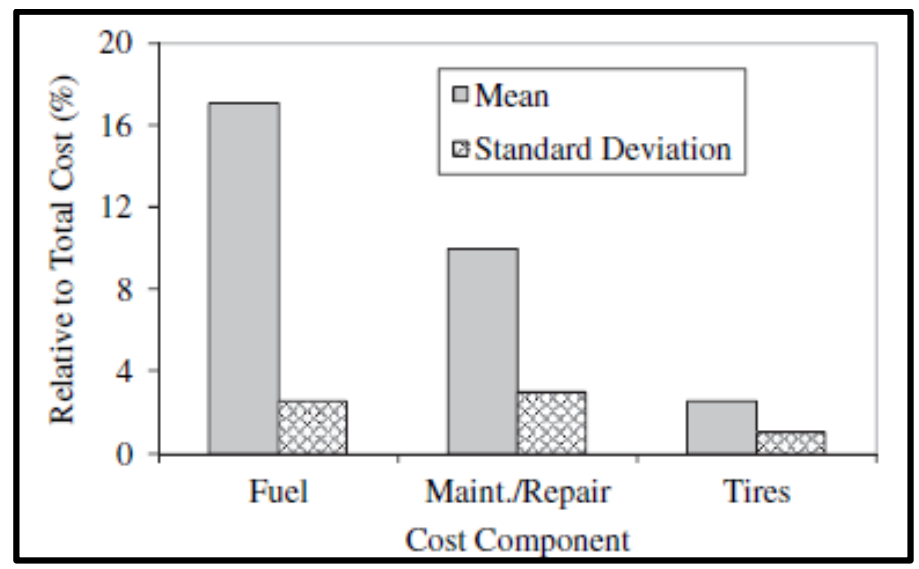

Figure 2-2 Relative vehicle operating costs for trucks (Chatti and Zaabar 2012)

\subsection{Chapter Summary}

This chapter provided an extensive literature review in the areas of: (1) vehicleroad interaction that mainly presented the influences of pavement characteristics on vehicle fuel consumption, (2) existing vehicle fuel consumption models, (3) an implementation of the optimization technique in highway construction programs, and (4) a basic knowledge about public benefits and public costs measured in highway transportation. This literature review shows the important gaps in the area of decision making for highway rehabilitation efforts and a pressing need for new research in order to facilitate environmental sustainability in transportation networks. The gaps reveal several research needs including: 
(1) developing new models for estimating vehicle fuel consumption and the savings in components of road user costs that are applicable to the transportation network analysis;

(2) developing robust models that are capable of analyzing and evaluating the impact of highway rehabilitation efforts on total energy consumption and net public benefits throughout transportation networks; and

(3) developing a multi-objective optimization model for planning highway rehabilitation efforts to search for and identify highway construction program(s) that are capable of simultaneously minimizing environmental impact and maximizing public benefits subject to budget constraints. 


\section{CHAPTER 3}

\section{MEASURING VEHICLE FUEL CONSUMPTION AND PUBLIC BENEFITS IN TRANSPORTATION NETWORKS}

\subsection{Introduction}

The main objective of this chapter is to develop mathematical modules for estimating vehicle fuel consumption and public benefits, which are related to the cost savings in three main components: (1) fuel consumption cost, (2) tire depreciation cost, and (3) repair and maintenance costs. However, fuel consumption cost can be easily calculated based on the fuel consumption rate and fuel price. Therefore, the estimating modules in this chapter are established based on the statistical approach to investigate the relationships between three components (vehicle fuel consumption, tire depreciation cost, and repair and maintenance costs) and main affecting factors. At the end, the mathematical estimating equations are proposed contributing an evaluation of the impact of rehabilitation efforts in highway transportation networks. In this chapter, the following sections focus on: (1) the concept of developing mathematical estimating modules for transportation network application; (2) the development of vehicle fuel consumption estimating module; (3) the development of tire depreciation cost rate estimating module; and (4) the development of repair and maintenance costs rate estimating module.

\subsection{Development of Mathematical Estimating Modules for Network Application}

Several studies have reported the impact of rehabilitation efforts in terms of economic and environmental perspectives, as main components in highway transportation rehabilitation decision making (Abaza 2002; de la Garza and Krueger 2007; Dhakal and

Oh 2011; Zhang et al. 2008; Zhang et al. 2012). Accordingly, it is very significant to 
evaluate the impact of rehabilitation programs for the entire transportation network in terms of energy consumption and public benefits. However, there are many challenges in an estimation of network energy consumption and public benefits. First, the energy estimating models used in many past studies require very comprehensive data (e.g. Lidicker et al. (2012), Wang et al. (2012)), which is mostly unavailable in the transportation pavement database. Second, in the public benefit estimation, some previous studies applied a constant value per one unit of vehicle for calculation (e.g. AASHTO (2010)), while some existing models require very intensive information (e.g. Chatti and Zaabar (2012)). These challenges reveal a deficiency of a robust approach that is able to contribute to the networklevel application.

As such, the estimations of energy consumption and public benefits require new and simple-yet-accurate equations to facilitate an analysis, since most of the existing models are not well-suited for the network-level calculation. In this chapter, the mathematical equations are developed to support the calculation of some input parameters' values necessary for the network analysis calculation. The equations are established by corresponding to three main components of vehicle operating costs - fuel consumption cost, tire depreciation cost, and repair and maintenance cost according to Chatti and Zaabar (2012).

As the estimation of network energy consumption highly correlates to total fuel usage of vehicles traveling in the transportation network, the fuel consumption rate is one of basic variables in the network energy consumption estimating model. Moreover, the fuel consumption, tire depreciation, and the repair and maintenance cost rate are directly related to the public benefits that can be evaluated in the terms of their cost savings as a result of 
rehabilitation implementation. The following sections describe the development of mathematical equations for estimating the fuel consumption rate, tire depreciation cost rate, and repair and maintenance costs rate in detail.

\subsection{Vehicle Fuel Consumption Estimating Module}

The objective of this section is to demonstrate the development of the mathematical equation for estimating the fuel consumption rate that is well-suited for the network analysis. A conceptual framework and development steps are firstly introduced, and then the statistically developed equations are presented afterwards.

\subsubsection{Model Development}

As aforementioned, a simple yet accurate equation is required to generate reliable calculation result. In this study, the HDM-4 model, which is the most popular model for vehicle operating cost estimation nowadays (Bennett and Greenwood 2003; Ferreira et al. 2011), is used for the development. Its recent version is currently implemented in many countries around the world, and there was an effort to calibrate the model to be practicable to the U.S conditions in Chatti and Zaabar (2012).

As the HDM4 model was developed for a comprehensive evaluation of pavement conditions on vehicle operating costs (Chatti and Zaabar 2012), there are some challenges for the network application. The model requires a large number of predictor variables (Dewan and Smith 2002), and very high-detailed level of input data (Zaabar and Chatti 2010). Additionally, some of the required variables are not available in most of transportation agencies' database systems (Dewan and Smith 2002). Therefore, to overcome these challenges for the network-level implementation, the main factors 
affecting vehicle fuel consumption are identified based on the past literature. Then, multiple regression method is employed for the analysis and the development of the mathematical equation. To this end, the estimating equation is proposed for quantifying the vehicle fuel consumption rate in such a way that facilitates the network application. The general framework of the equation development can be represented as shown in Figure 3-1.

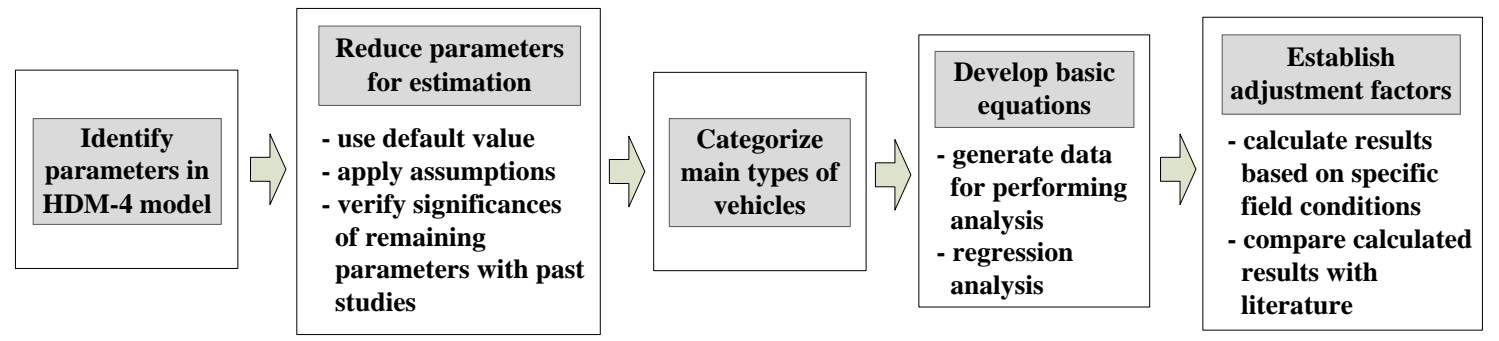

Figure 3-1 General flowchart for the equation development

The processes in developing the equations can be categorized into five main groups. The description of each detailed step is given as follows.

(1) Identify Parameters in the HDM-4 Model

All parameters in the HDM-4 model are investigated and they can be summarized as shown in Figure 3-2. These variables are related to three types of the power that vehicles have to overcome for moving, which are (1) power required for engine accessories; (2) power required to overcome internal engine friction; and (3) power required to overcome traction forces. The examples of the parameters are fuel consumption at idling, idle engine speed, gradient, curvature radius, and international roughness index. 


\section{(2) Reduce Parameters Used for Estimation}

As aforementioned, the calibrated HDM4 model from Chatti and Zaabar (2012) is employed for this section. To develop the network-application fuel consumption estimating equations, some parameters should be omitted from the development of equations, as most of them are not available in the typical transportation database. The challenging task is to establish the estimating equations in such a way that complies with the data accessibility and facilitates the network analysis. To overcome this challenge, three major steps are executed, as follows:

(2.1) Use default data defined in the HDM-4 model

The default data is simply used if any parameters have them defined in Chatti and Zaabar (2012). All lists of this type of variables can be summarized as shown in Figure 3-2.

(2.2) Apply assumptions from the field test and past literature

There are two solutions adopted here in applying assumptions to reduce the number of parameters in the HDM-4 model. First, the actual conditions from the field trails performed in the reference are taken for the analysis. The parameters borrowing the information from the field test conditions in this section are mean profile depth values with the range from 0.2 to $2.7 \mathrm{~mm}$, and gradient ranging from $-3.4 \%$ to $3.1 \%$.

Second, some relevant references are further investigated if a parameter is not applicable to the first solution. Benkleman Beam rebound deflection is presumed as $0.75 \mathrm{~mm}$. Also, vehicle acceleration is assumed to be $0.64 \mathrm{~m} / \mathrm{s}^{2}$ as modified from Brooks (2012). 
(2.3) Verify the significances of remaining parameters with past studies

After applying the default values and assumptions in steps 2.1 and 2.2, the estimating equations can be developed in terms of three remaining parameters - vehicle type, vehicle speed, and international roughness index. From the literature, several past studies have declared the impact of vehicle type on fuel consumption (AASHTO 2010; Barnes and Langworthy 2004; Chatti and Zaabar 2012; Taylor and Patten 2006; Watanatada et al. 1987; Yu and Lu 2012). This supports that the vehicle classification should be taken into account for the fuel consumption estimation. In addition to vehicle type, vehicle speed is significant, as it is still taken into consideration in many existing models, such as AASHTO (2010), and HDM4 (Chatti and Zaabar 2012).

Additionally, many studies have strived to observe the impact of pavement conditions on fuel consumption. Pavement conditions are commonly measured in terms of the international roughness index (IRI), which was developed by the World Bank (Sayers and Karamihas 1998). The significance of pavement roughness has been acknowledged in many pieces of published literature (Akbarian et al. 2012; Akcelik and Besley 2003; Amos 2006; Epps et al. 1999; Yu and Lu 2012; Zaabar and Chatti 2010; Zhang et al. 2009). Towards this end, a massive number of research studies reach a similar conclusion, which explicitly affirms the important effect of IRI on vehicle fuel consumption.

As a result, three main factors - vehicle type, vehicle speed, and pavement roughness - are considered as basic parameters in establishing the mathematical equations in order to advocate for further analysis at the network-level to estimate the vehicle fuel consumption rate, and therefore total network energy consumption. The analysis process 
for considering the basic parameters through steps 1 and 2 can be intensively illustrated as shown in Figure.3-2.

\section{All Required Parameters in HDM-4 Model}

\begin{tabular}{|c|}
\hline Default data \\
\hline Fuel consumption at idling \\
Engine efficiency \\
Rated engine power \\
Engine horsepower \\
Excess fuel consumption due to congestion \\
Power required to overcome internal engine friction \\
Ratio of engine and accessories drag to rated engine power when traveling at $100 \mathrm{~km} / \mathbf{h}$ \\
Percentage of the engine and accessories power used by the engine \\
Engine speed at 100 km/h \\
Idle engine speed \\
Drive-train efficiency factor \\
Drag coefficient \\
Frontal area \\
Mass density of the air \\
Vehicle weight \\
Curvature radius \\
Number of wheels \\
Rolling resistance tire factor \\
Wheel diameter \\
Tire type \\
\hline
\end{tabular}

Figure 3-2 List of basic parameters in the equation development 


\section{(3) Categorize Main Types of Vehicles in the HDM-4 Model}

Since the vehicle class in the HDM-4 model is very comprehensively identified, this is not practicable to the network application. To overcome this challenge, the fuel consumption rates of all vehicle classes are calculated by assuming specific values of vehicle speed and pavement roughness. The vehicle classes are then grouped based on the calculated fuel consumption rates into three main groups - passenger car, light-duty truck, and heavy-duty truck.

\section{(4) Develop Basic Equations for Estimation}

The main objective of this step is to develop the mathematical equations that are able to provide the reliable calculation results of the vehicle fuel consumption rate with respect to three main factors - vehicle type, vehicle speed, and pavement roughness. To address this objective, multiple regression analysis is employed since it has a powerful capability to accurately predict the unknown value of a variable (called dependent variable) from the known value of multiple variables (called independent variables) (Higgins 2005). All activities in this step can be grouped into two main groups as follows.

\section{(4.1) Generate data for performing analysis}

This step is about generating the data that will be used as input variables in regression analysis. The first activity is entering all equations from the HDM4 fuel consumption model in Chatti and Zaabar (2012) into a spreadsheet. Then, the values of related parameters from the default values and the assumptions are substituted in the equations. After that, the data are generated by ranging the value of vehicle speed from 8-

$120.7 \mathrm{~km} / \mathrm{h}(5-75 \mathrm{mph})$ with an increment of $8 \mathrm{~km} / \mathrm{h}(5 \mathrm{mph})$, and varying the value of 
pavement roughness (in terms of IRI) based on the typical values from 0 to $5 \mathrm{~m} / \mathrm{km}$ (Wang 2013) by the increment of $1 \mathrm{~m} / \mathrm{km}$.

\section{(4.2) Perform regression analysis}

This step starts from plotting the curves to check the relationships between each independent variable and the dependent variable. This helps the analyst to select the most appropriate mathematical form. Then, regression analysis is run by using the toolbox in the SPSS software. Finally, the R-square values of the equations are checked to observe the effectiveness in predicting the dependent variable from independent variables. If the Rsquare is close to 1 or meets the analyst's criteria, then interpret the SPSS result. The mathematical equation can be constructed by considering the regression coefficients and their relationships to the relevant variables.

\section{(5) Establish Adjustment Factors}

The main objective of this step is to verify the results calculated based on the developed basic equations with the predicted fuel consumption rates from the testification of the field data test mentioned in Chatti and Zaabar (2012). This step compares the results from the regression model with the values in the literature, and then proposes the adjustment factors for correcting the calculated fuel consumption rate to be more realistic and accurate. Two steps are mainly performed herein: (1) calculate results based on specific field conditions mentioned in the literature; and (2) compare calculated results with the values in the literature and propose adjustment factors. The next section will explain the detail of regression analysis and the development of the equation. 


\subsubsection{Mathematical Equations for Estimating Fuel Consumption Rate}

Considering the relationship plots between each main factor (as the independent variable) and the fuel consumption rate (as the dependent variable), a well-suited mathematical model can be identified for regression analysis. Figure 3-3 illustrates the example of the relationship plots for a passenger car. It shows an incremental linear relationship between pavement roughness and the fuel consumption rate (see figure 3-3(a)). In comparison, the quadratic function is expressed in the vehicle speed-fuel consumption plot, as shown in figure 3-3(b). This pattern shows the minimum on the curve and different slopes for the downward and upward directions. Therefore, the mathematical equations should be developed by separating for two speed ranges corresponding to the vertex point. For instance, calculating the fuel consumption rate for a passenger car can be performed by using two equations, depending on the range of average speed of vehicles traveling on a road, when vehicle speed is: (1) less than or equal $64.4 \mathrm{~km} / \mathrm{h}$ (40 mph); and (2) more than $64.4 \mathrm{~km} / \mathrm{h}$ (40 mph). Table 3-1 presents the mathematical equations and the adjustment factors for estimating the fuel consumption rate for three main vehicle types. It is worth noting that all equations have the r-square values close to 1 , which means that they are able to efficiently predict the results.

From the equations, the fuel consumption rate (in $\mathrm{mL} / \mathrm{km}$ ) can be predicted if vehicle speed, vehicle type, and IRI are known. Vehicle speed (S) and IRI are in $\mathrm{km} / \mathrm{h}$ and $\mathrm{m} / \mathrm{km}$, respectively. The vehicle fuel consumption rate calculated from this section will be entered in the network energy consumption estimating model that will be introduced in the chapter 4. 


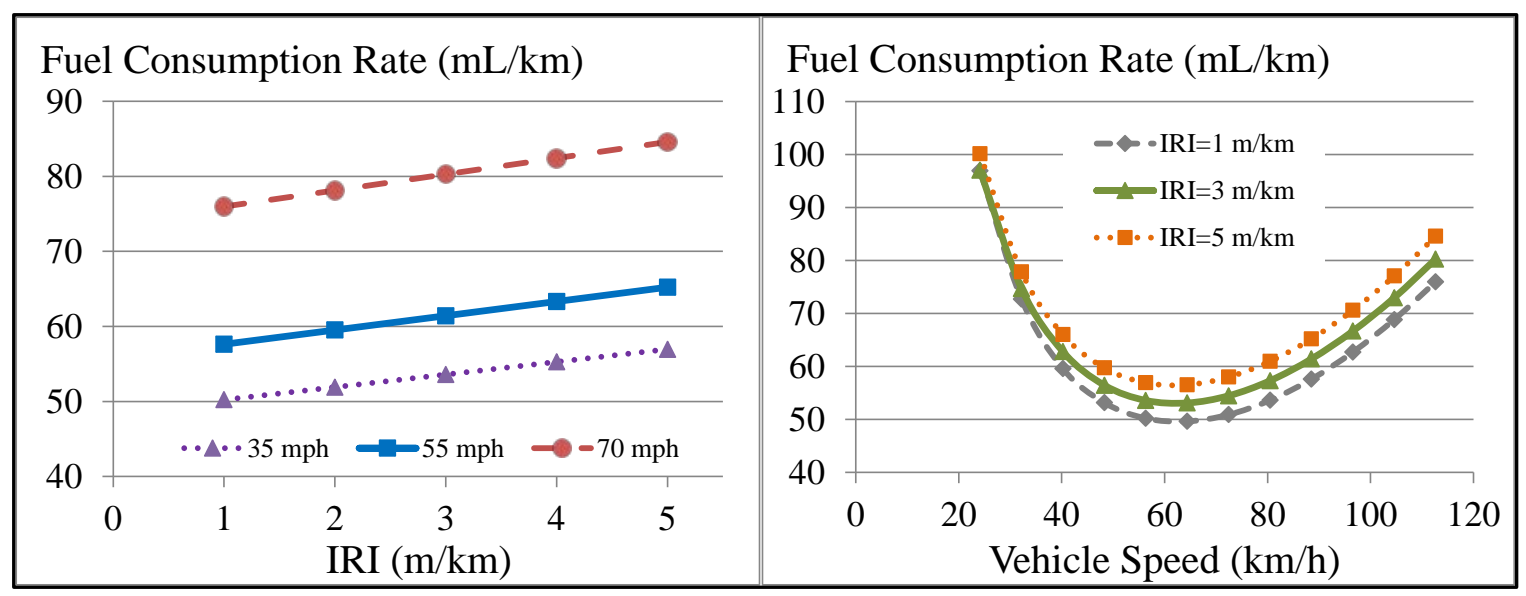

(a)

(b)

Figure 3-3 Relationships between main factors and fuel consumption rate for passenger cars

(a) Pavement roughness vs. fuel consumption rate;

(b) Vehicle speed vs. fuel consumption rate

Table 3-1 Vehicle energy consumption estimating equations

\begin{tabular}{|c|c|c|c|c|}
\hline $\begin{array}{c}\text { Vehicle } \\
\text { Type }\end{array}$ & $\begin{array}{c}\text { Vehicle } \\
\text { Speed } \\
\text { km/h }\end{array}$ & Equation & $\begin{array}{c}\text { Adj. } \\
\text { Factor }\end{array}$ & $\mathbf{R}^{2}$ \\
\hline \multirow{2}{*}{$\begin{array}{c}\text { Passenger } \\
\text { Car }\end{array}$} & $\leq 64.4$ & FR $=1.221 \mathrm{IRI}-13.066 \mathrm{~S}+0.134 \mathrm{~S}^{2}$ & 1.274 & 0.923 \\
\cline { 2 - 5 }+64.4 & $\mathrm{FR}=1.917 \mathrm{IRI}+0.567 \mathrm{~S}+7.783$ & 1.294 & 0.956 \\
\hline $\begin{array}{c}\text { Light- } \\
\text { Duty } \\
\text { Truck }\end{array}$ & $\leq 48.3$ & $\mathrm{FR}=1.295 \mathrm{IRI}-18.144 \mathrm{~S}+0.236 \mathrm{~S}^{2}$ & 1.206 & 0.957 \\
\cline { 2 - 5 }+48.3 & $\mathrm{FR}=1.481 \mathrm{IRI}+1.093 \mathrm{~S}+33.021$ & 1.317 & 0.933 \\
\hline $\begin{array}{c}\text { Heavy- } \\
\text { Duty } \\
\text { Truck }\end{array}$ & $\leq 48.3$ & $\mathrm{FR}=3.994 \mathrm{IRI}-23.831 \mathrm{~S}+0.315 \mathrm{~S}^{2}+625.631$ & 1.257 & 0.953 \\
\cline { 2 - 5 } & $>48.3$ & $\mathrm{FR}=4.373 \mathrm{IRI}+2.37 \mathrm{~S}+61.946$ & 1.435 & 0.958 \\
\hline
\end{tabular}

\subsection{Tire Depreciation Cost Rate Estimating Module}

This section presents the development of mathematical equation for estimating the tire depreciation cost rate, which is one of input in evaluating the public benefits on transportation networks. It consist of two main subsections: (1) model development that 
describes the basic framework and assumptions used in developing the estimating module, and (2) mathematical equations and the relationships between the cost rate and main affecting factors. The detail of each subsection is given as follows.

\subsubsection{Model Development}

From the literature, the most updated tire depreciation model is found as a part of the HDM4 in Bennett and Greenwood (2003). However, this recent version was later modified by calibrating to the U.S. conditions in Chatti and Zaabar (2012). Accordingly, the calibrated model is adopted in this study for developing the mathematical equations that are simple and reliable enough for implementing at the network-level analysis.

Similar to the fuel consumption rate, the HDM4 model has some challenges that have to be overcome for the transportation network application. Very detailed information is needed as the input variable in the calibrated HDM4 model. Unfortunately, some variables are not currently measured and collected by transportation agencies. To this end, the existing studies are compiled to explore the main factors that significantly affect tire deprecation. Multiple regression analysis is subsequently applied to establish numerical equations that are able to serve estimation at the network level. The process for the equation development is similar to the fuel consumption's. Therefore, this section will omit a detailed explanation of how to reduce the parameters in the HDM-4 model since it has a similar concept as in the fuel consumption section. However, the following paragraph will verify the significances of three remaining basic parameters necessary for the development of equation.

The past literature has presented the importance of vehicle type to tire depreciation cost. It shows that a large vehicle tends to have higher tire consumption rate than a smaller 
vehicle (AASHTO 2010; Chatti and Zaabar 2012; Zaniewski et al. 1982). In addition to vehicle type, pavement roughness has a major impact on tire depreciation (Barnes and Langworthy 2004; Chatti and Zaabar 2012). The supporting reason is the effect of pavement roughness on the rolling resistance force. As the IRI value increases, rolling resistance grows larger, and this affects the amount of tire worn since tire wear is directly proportional to the rolling resistance force. Additionally, it appears that the impact of pavement roughness on tire depreciation becomes greater while increasing vehicle speed (Chatti and Zaabar 2012). As a result, these three main factors found from the literature vehicle type, vehicle speed, and pavement roughness - are taken into account for developing the numerical equations to estimate tire depreciation cost rate that is able to facilitate the network-level calculation.

\section{Assumptions for Equation Development}

Similar to fuel consumption, some assumptions need to be addressed before developing the mathematical form. To receive the tire depreciation cost rate, the calibrated HDM4-based model in Chatti and Zaabar (2012) is modified to incorporate only three main factors mentioned in the previous section. To accomplish this task, similar assumptions applied for fuel consumption are also used in this section. However, there are some differences in the assumptions used for the truck's analysis, as follows.

(1) The gradient adopted from the historical truck tire wear data ranges from 0.5\% to $0.5 \%$, as mentioned in Chatti and Zaabar (2012).

(2) The mean profile depth for truck testing is not stated anywhere in the reference. Therefore, it is assumed to be zero in this calculation part. 


\subsubsection{Mathematical Equations for Estimating Tire Depreciation Cost Rate}

In consideration of the relationships between the tire depreciation rate and main affecting factors (see Figure 3-4), vehicle speed has continuously curve relationships to tire depreciation rate while linear pattern appears between IRI and tire depreciation rate. Consequently, the regression analysis is performed by mathematically assuming the polynomial function to develop the tire depreciation cost rate estimating equations that are able to be effectively support the network analysis.

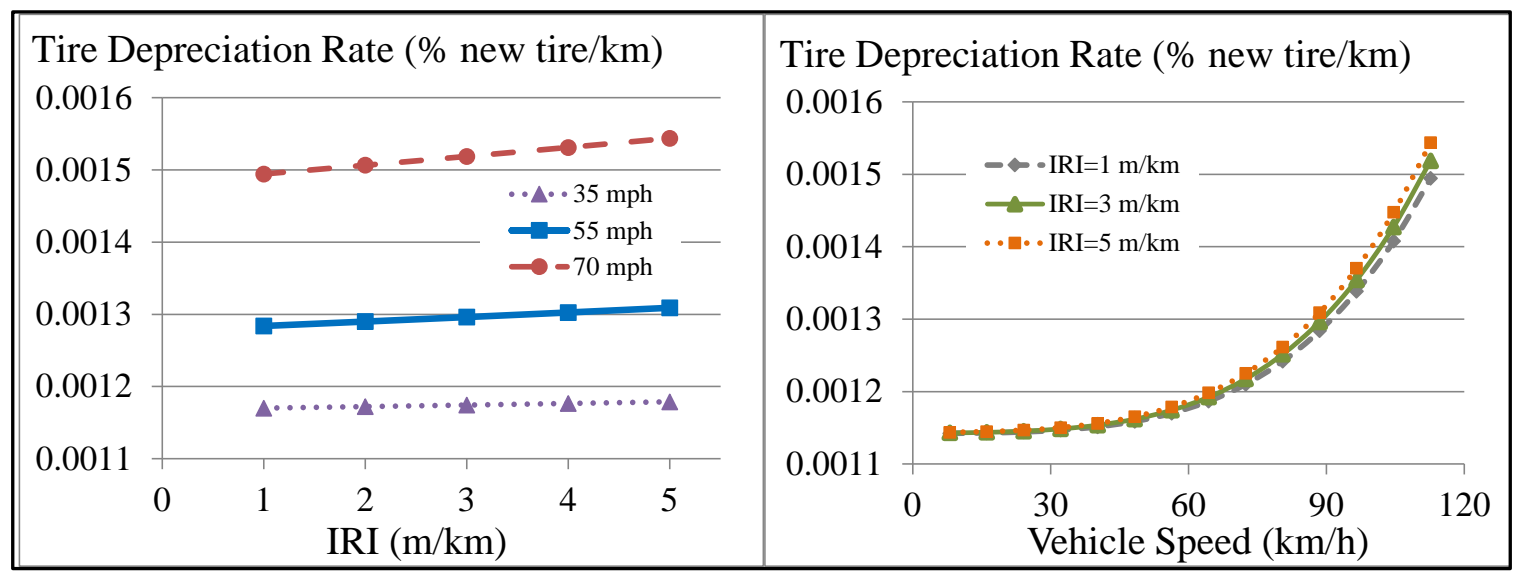

(a)

(b)

Figure 3-4 Relationships between main factors and tire depreciation rate for passenger cars

(a) Pavement roughness vs. tire depreciation rate;

(b) Vehicle speed vs. tire depreciation rate

Table 3-2 Vehicle tire depreciation estimating equations

\begin{tabular}{|c|c|c|c|}
\hline Vehicle Type & Equation & $\begin{array}{c}\text { Adj. } \\
\text { Factor }\end{array}$ & $\mathbf{R}^{2}$ \\
\hline Passenger Car & $\mathrm{DR}=\left(3.979 \times 10^{-6}\right) \mathrm{IRI}+\left(3.198 \times 10^{-6}\right) \mathrm{S}+0.001$ & 1.071 & 0.795 \\
\hline $\begin{array}{c}\text { Light-Duty } \\
\text { Truck }\end{array}$ & $\mathrm{DR}=\left(1.436 \times 10^{-6}\right) \mathrm{IRI}+\left(3.178 \times 10^{-6}\right) \mathrm{S}+0.001$ & 1.547 & 0.795 \\
\hline $\begin{array}{c}\text { Heavy-Duty } \\
\text { Truck }\end{array}$ & $\mathrm{DR}=\left(9 \times 10^{-7}\right) \mathrm{IRI}+\left(1.308 \times 10^{-6}\right) \mathrm{S}$ & 1.222 & 0.809 \\
\hline
\end{tabular}


Table 3-2 presents the mathematical equations for estimating the tire depreciation rate in terms of three main factors - vehicle type, vehicle speed, and pavement roughness. The equations are proposed for three main vehicle types - passenger car, light-duty truck, and heavy-duty truck. All developed equations are able to efficiently predict the reliable results with the r-square values close to 1 . From the equations, the tire depreciation rate can be obtained if vehicle speed, vehicle type, and IRI are known. Vehicle speed and IRI are in $\mathrm{km} / \mathrm{h}$ and $\mathrm{m} / \mathrm{km}$, respectively. The rate calculated from this section will be converted to the cost by multiplying with the cost per tire and number of vehicle wheels, and then placed into the public benefit estimating model that will be introduced in the chapter 4 .

\subsection{Repair and Maintenance Costs Rate Estimating Module}

This section demonstrates the development of the repair and maintenance costs rate estimating module, which is able to facilitate the transportation network calculation. Similar to the two previous sections, the detail of this module is categorized and presented in to two main sections: model development and mathematical equations, as follows.

\subsubsection{Model Development}

In this study, the model developed in Chatti and Zaabar (2012) is adopted for estimating vehicle repair and maintenance costs rate. This developed model is the combination of two repair and maintenance costs models that are claimed as the two most potential models according to Chatti and Zaabar (2012): (1) the HDM4 model, and (2) the relevant model from the Texas Research and Development Foundation (TRDF) study (Zaniewski et al. 1982). In order to develop the equation for estimating the repair and maintenance costs rate that is able to be competently applicable to the network analysis, 
the past literature studies are observed for identifying the main relevant factors. The multiple regression approach is then used to construct the mathematical equations.

A significant number of past studies have revealed the effect of vehicle classification on repair and maintenance costs. These studies presented different repair and maintenance costs on a variety of vehicle types (AASHTO 2010; Barnes and Langworthy 2004; Bennett and Greenwood 2003; Chatti and Zaabar 2012; Zaniewski et al. 1982). Moreover, Chatti and Zaabar (2012) highlighted the influence of IRI and vehicle speed on repair and maintenance costs. This existing study showed an increase in the impact of IRI on repair and maintenance costs while vehicle speed increases. The results from other references (e.g. Zaniewski et al. (1982)) also confirmed the significance of vehicle operating speed on repair and maintenance costs. The costs tend to grow, corresponding to a higher vehicle speed and larger vehicle size. To this end, three main factors - vehicle type, vehicle speed, and IRI - are considered in developing the equation for quantifying the repair and maintenance costs rate in this study.

\subsubsection{Mathematical Equations for Estimating Repair and Maintenance Costs Rate}

The plot in Figure 3-5 (b) shows that vehicle speed expresses a nearly linear relationship with the repair and maintenance costs rate. In contrast, a continuously curve trend is established between the IRI and the repair and maintenance costs (see Figure 3-5 (a)) with no effect on the change of the costs at a low level of roughness (at IRI $\leq 3 \mathrm{~m} / \mathrm{km}$ ). The multiple regression analysis is used to develop the equation for estimating the repair and maintenance costs rate by assuming the polynomial function. Some related parameters for estimation are reduced and then only two main variables, IRI and vehicle speed, are left to incorporate for the equation establishment. 


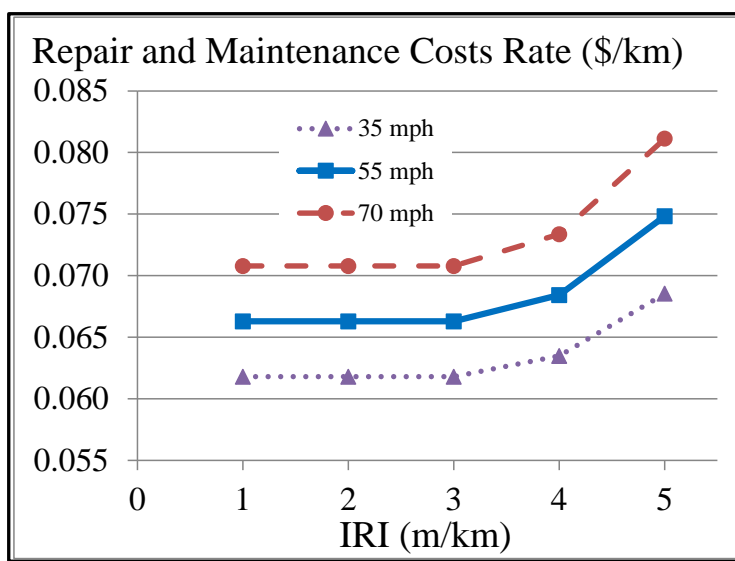

(a)

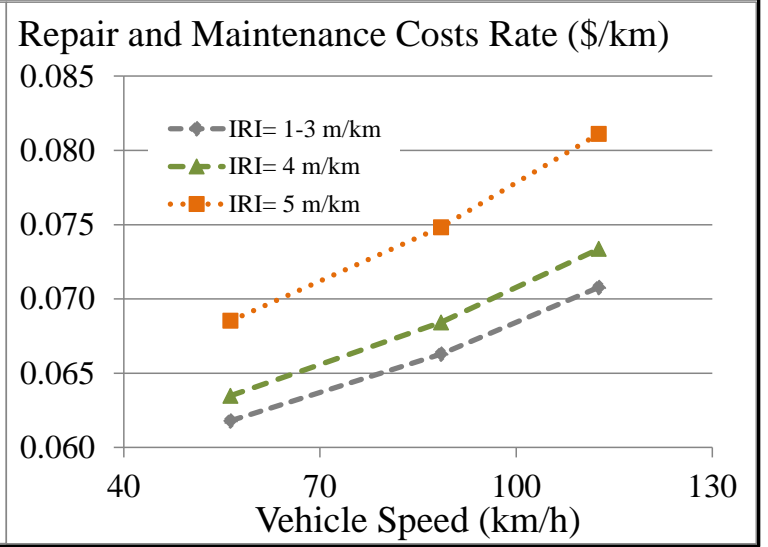

(b)

Figure 3-5 Relationships between main factors and repair and maintenance costs for passenger cars

(a) Pavement roughness vs. repair and maintenance costs rate;

(b) Vehicle speed vs. repair and maintenance costs rate

Table 3-3 Vehicle repair and maintenance estimating equations

\begin{tabular}{|c|c|c|}
\hline Vehicle Type & Equation & $\mathbf{R}^{2}$ \\
\hline Passenger Car & $\mathrm{MR}=0.003 \mathrm{IRI}+0.00019 \mathrm{~S}+0.044$ & 0.847 \\
\hline Light-Duty Truck & $\mathrm{MR}=0.008 \mathrm{IRI}+0.000377 \mathrm{~S}+0.049$ & 0.826 \\
\hline Heavy-Duty Truck & $\mathrm{MR}=0.018 \mathrm{IRI}-0.0174 \mathrm{~S}+0.000125 \mathrm{~S}^{2}+0.723$ & 0.922 \\
\hline
\end{tabular}

Repair and maintenance costs can be calculated by using an equation associated with vehicle type, as shown in Table 3-3. The repair and maintenance costs rate depends on type of vehicle, vehicle speed $(\mathrm{km} / \mathrm{h})$, and IRI $(\mathrm{m} / \mathrm{km})$. Similar to fuel consumption and tire depreciation, all equations for repair and maintenance costs are developed with the rsquare close to 1 in order to assure if the calculated result can be a good representative and applicable to the network-level analysis. The repair and maintenance costs rate estimated 
from the developed equation in this section will be used in the public benefit estimating model that will be introduced later in the chapter 4 .

\subsection{Chapter Summary}

This chapter presented the development of novel estimating models to support an evaluation of impacts resulting from rehabilitation efforts on the transportation network, since the existing models are not well-suited for the network-level application regarding the limitation of data availability. The developed models are proposed with mathematical equations that are capable of estimating vehicle fuel consumption rate, tire depreciation cost rate, and repair and maintenance costs rate, in which the last two components are directly related to the savings in road user costs for an estimation of public benefits. In this study, the models from the National Cooperative Highway Research Program (NCHRP) report 720 (Chatti and Zaabar 2012) were adopted and integrated with the statistical technique to establish the mathematical equations that is able to effectively facilitate the transportation network estimation. Accordingly, the estimating models are developed in form of mathematical equations that show the relationships between vehicle fuel consumption rate, tire depreciation cost rate, and repair and maintenance costs rate, and their main affecting factors, which include vehicle speed, vehicle type, and pavement conditions. The models formulated in this chapter will be useful for estimating some parameters necessary in the evaluation of highway rehabilitation efforts on transportation networks that will be later introduced in the chapter 4 . 


\section{CHAPTER 4}

\section{EVALUATING THE ECONOMIC AND ENVIRONMENTAL IMPACTS OF REHABILITATION EFFORTS ON TRANSPORTATION NETWORKS}

\subsection{Introduction}

The main objective of this chapter is to present the development of a new model for evaluating the economic and environmental impacts on transportation networks as a result of decision making in highway rehabilitation efforts. The model is capable of: (1) identifying candidate rehabilitation treatment alternatives to a deteriorating pavement; (2) evaluating and forecasting the impact of rehabilitation treatments on pavement performance; (3) estimating total energy consumption throughout the entire network as a result of highway rehabilitation decisions; (4) estimating the public costs as a result of the vehicle travel-delay from the speed reduction during the construction operations, and (5) evaluating the impact of rehabilitation decision making on public benefits as the expected savings in road user costs after rehabilitation. In this chapter, each part of the developed model is described in order along with its conceptual framework and calculation algorithm. The application example is also analyzed to illustrate the performance and capabilities of the developed model in the last section of this chapter.

\subsection{Model Development}

It is very significant for transportation planning agencies to understand the impact of rehabilitation efforts on overall network performance to produce the effective rehabilitation plan. Several studies have reported the impact in terms of economic and environmental perspectives, as main components in considering highway maintenance and rehabilitation programs (Abaza 2002; de la Garza and Krueger 2007; Dhakal and Oh 2011; 
Zhang et al. 2008; Zhang et al. 2012). Economic assessment has been analyzed as public cost and benefits in numerous research studies (de la Garza et al. 2011; Irfan et al. 2012; Mbwana and Turnquist 1996; Orabi and El-Rayes 2011). For environmental sustainability, as the concept of environmental awareness gains interest widely, it began to be the focus of many researchers in the past decade. Recently, energy consumption in transportation systems has therefore been the main focus in many studies (e.g. Dhakal and Oh (2011), Zhang et al. (2008)). Considering the increasing research interest and huge user costs and fuel usage, it becomes the significance to evaluate the impacts of rehabilitation programs for the entire network on three main components: (1) energy consumption, (2) public cost, and (3) public benefits.

The estimation of energy consumption in highway transportation was acknowledged in several research studies. However, there are some challenging tasks that can enhance the body of knowledge in this area. First, the energy estimating model used in some studies requires very comprehensive data (e.g. Lidicker et al. (2012),Wang et al. (2012)), which is mostly unavailable in the transportation pavement database. Second, energy consumption is not the main focus in some existing studies, such as Cass and Mukherjee (2011), Lidicker et al. (2012), Zhang et al. (2008), and Zhang et al. (2012). This prevents researchers from conducting a thorough investigation of the impact of transportation on energy consumption. Third, most energy-related studies have been performed at the project level. Although some environment-related studies (e.g. Zhang et al. (2012)) were proposed for transportation network evaluation, energy usage is still not a major concern in the studies. As a result, the study about the impact of the rehabilitation plan on the transportation network is pressingly needed to fulfill the current gap in this 
research domain. Additionally, the estimating model should be simple yet reliable to implement for the network-level assessment.

Public cost has been measured in terms of user costs, which generally include the cost of travel delay, and increased vehicle operating costs from work-zone speed reduction (Irfan et al. 2012; Wilde et al. 1999). User cost is a necessary component to be included in planning highway rehabilitation efforts. It has been investigated in a large number of research studies, such as Fwa and Sinha (1991), Hong and Prozzi (2013), Irfan et al. (2012), Lamptey et al. (2008), and Zhang et al. (2010).

Public benefits have been considered in the area of highway planning as shown in several existing studies. However, there is room for improvement that can be accomplished. Some previous studies applied a constant value per one unit of vehicle for calculation (e.g. AASHTO (2010)), while some existing models require very intensive information (e.g. Chatti and Zaabar (2012)). In addition, a robust approach contributing to the network-level application is deficient. Accordingly, there is a pressing need to address the critical research gaps mentioned above by developing a novel model for evaluating the impact of highway rehabilitation implementation on three main components: (1) energy consumption, (2) public cost, and (3) pubic benefits for the entire transportation network.

To this end, this chapter presents the development of a new model that is capable of evaluating the impact of highway rehabilitation decision making on transportation networks. This model is composed of five different modules: (1) rehabilitation treatment alternative identification module; (2) network pavement performance evaluating module; (3) network energy consumption estimating module; (4) public cost estimating module; 
and (5) public benefits estimating module. Figure 4-1 illustrates the model framework and the detail of each module will be provided in the following sections.

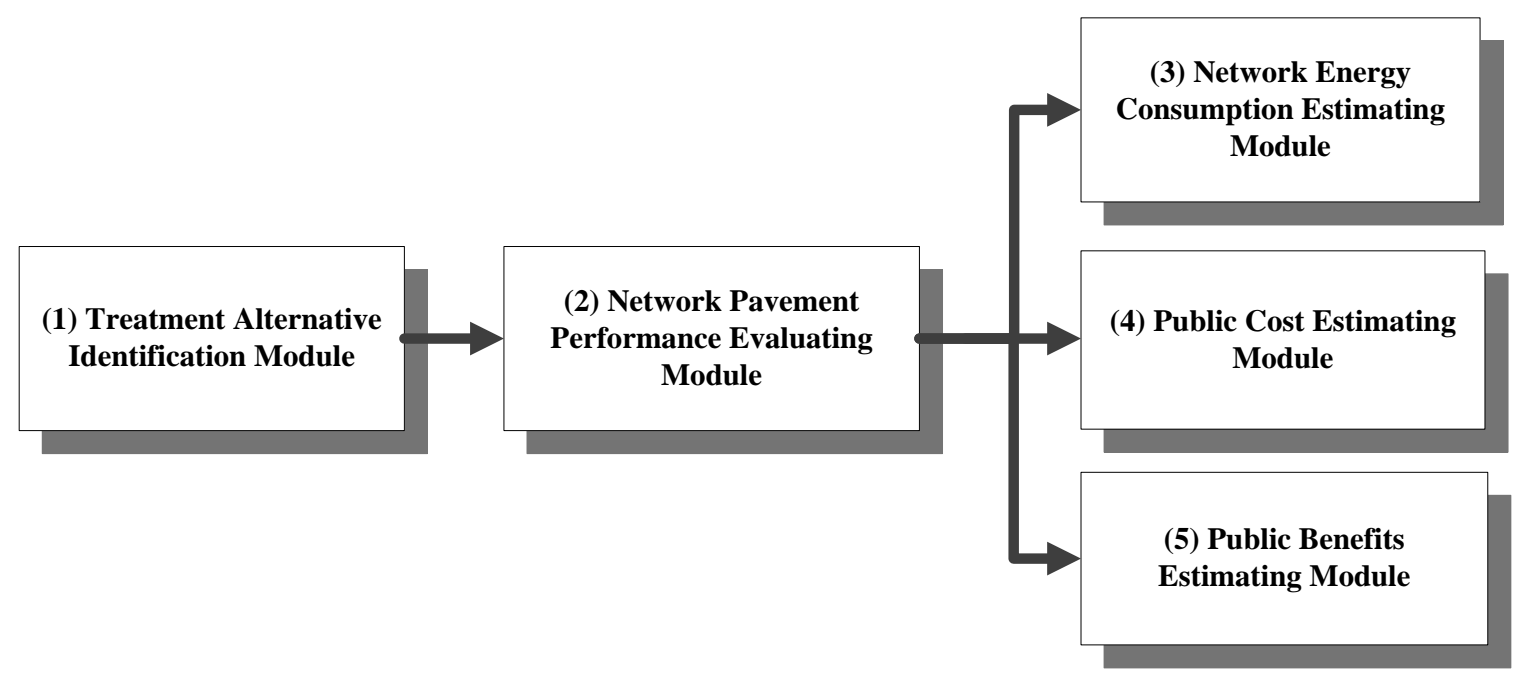

Figure 4-1 Economic and environmental impacts evaluating model

\subsection{Rehabilitation Treatment Alternative Identification Module}

The highway rehabilitation program typically designates which treatment should be implemented and when the treatment should be performed on deteriorating pavement. In this section, the rehabilitation treatment alternative identification module is established to be a guideline for decision makers in selecting the type of application treatment and application time. Theoretically, treatment timing can depend on age-based or performancebased thresholds (Labi and Sinha 2003; Lamptey et al. 2005). Age-based strategy is related to the pavement lifetime, in which rehabilitation intervention treatment will be carried out at the predefined timing interval. The application timing can be identified in several ways, such as an application frequency or a recommended design life of a treatment, as in several past studies and reports, for example, Geoffroy (1996), Hicks et al. (1999), Lamptey et al. (2005), and Zimmerman et al. (2002). However, the age-based thresholds may cause an 
inappropriate application of rehabilitation treatment, as the pavement may be preserved or rehabilitated at either too-early or too-late stages resulting from the uncertainties in pavement deterioration (Khurshid 2010).

On the other hand, the performance-based strategy uses the conditions of pavement performance as a threshold for rehabilitation implementation. A specific treatment will be applied on a road when the pavement conditions reach a certain threshold value. Pertaining to pavement, the performance can be indexed to measure the structural deficiency, such as Pavement Condition Rating (PCR), Rutting Index (RI), and International Roughness Index (IRI) (Khurshid 2010). In this study, IRI is used as a pavement performance indicator because it is a well-established and the most widely-used indicator representing pavement roughness that is utilized worldwide in many transportation agencies (Gillespie 1992; Paterson and Watanatada 1985; Sayers et al. 1986; Sayers and Karamihas 1998). Also, IRI is referred to as the "single best predictor" describing driver perception in road roughness and driving quality (Shafizadeh et al. 2002).

With respect to drawbacks of the age-based thresholds, the performance-based strategy is adopted in this study. Therefore, the thresholds here in this study will be mentioned as what level of IRI should be identified to carry out a specific rehabilitation treatment. The literature review revealed that various researchers utilized the performancebased strategies in their studies, including AI\&T (2006), Hall et al. (2001), Hicks et al. (1999), Mn/DOT (2001), Wade et al. (2001), and Zimmerman and Peshkin (2004). However, the guidance stated in those studies may be established based on different practical objectives. For instance, Zimmerman and Peshkin (2004) generally present the treatment guideline associated with a treatment category rather than a specific type of 
treatment by using Pavement Condition Index (PCI), while Wade et al. (2001) introduce the treatment selection scheme with the integration of various pavement indices in selecting a particular type of treatment. Accordingly, transportation planners can decide appropriate guidelines that are best-fitted to their planning strategies and the availabilities of pavement performance indices in transportation system database.

In this study, existing literature works are compiled to generate the rehabilitation treatment alternative module. The steps and the relevant literature for the module development are illustrated in Figure 4-2. At the beginning, the guideline flowchart representing pavement condition states and highway maintenance activities from de la Garza and Krueger (2007) are adopted for selecting appropriate rehabilitation treatment. This flowchart shows the pavement performance rating as a descriptive term with five levels of pavement performance - very good, good, fair, poor, and very poor conditions and the potential treatments with respect to the current conditions of pavement. However, to evaluate the impact of rehabilitation decisions on network pavement, the magnitude of impacts due to different treatments has to be recognized. The performance jump and posttreatment performance models developed in Irfan (2010) are used in this study to calculate the expected effect of rehabilitation treatment on pavement performance. Integrating these two past studies creates two challenges: (1) a correlation between the descriptive pavement rating and IRI value; and (2) a compatibility in the definition of treatment among the studies. 


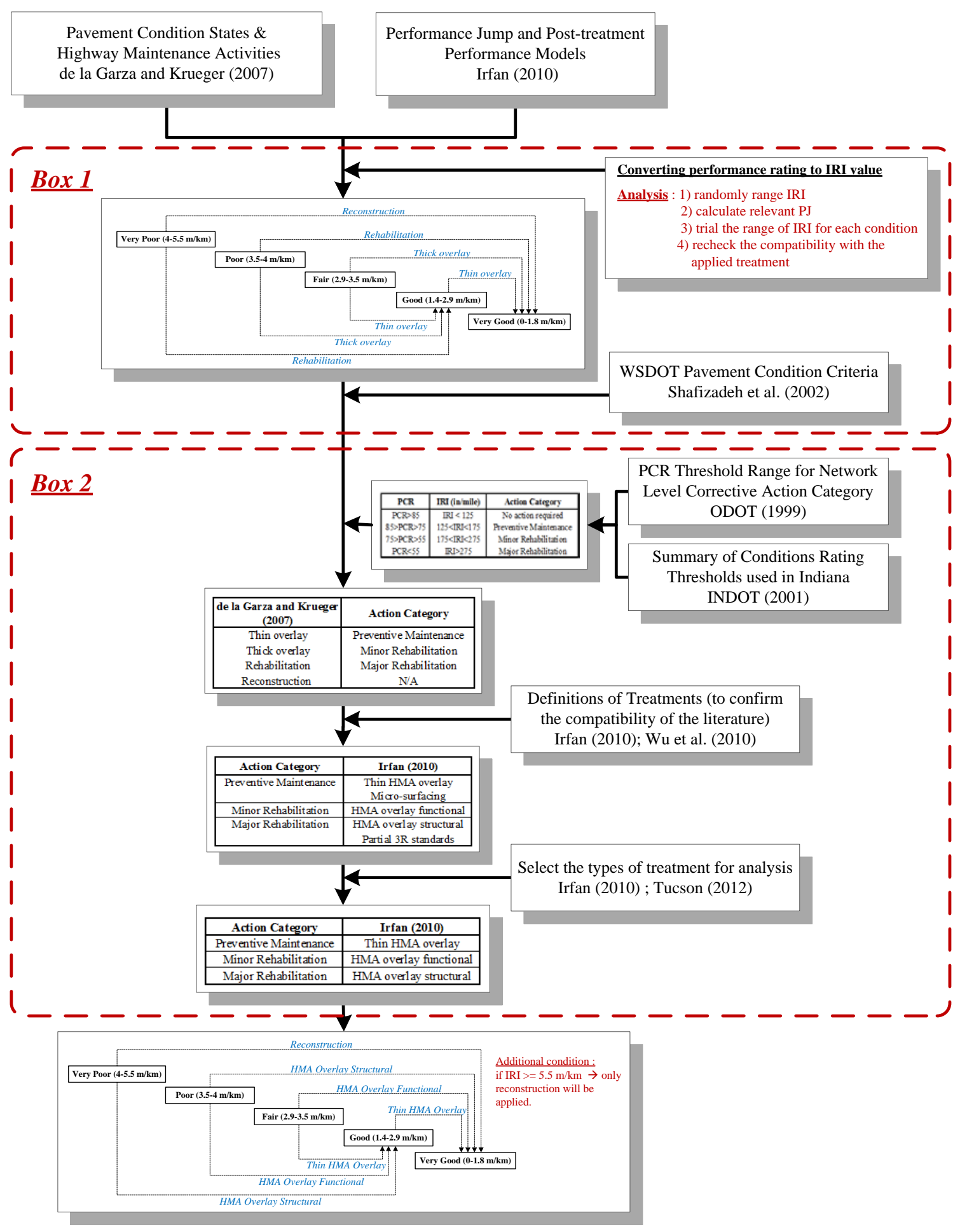

Figure 4-2 Development of rehabilitation treatment alternative identification module 
To deal with the first challenge (see Box 1 in Figure 4-2), the numerical adjustment is executed to find the appropriate range of IRI for each pavement performance rating. This range is established to create the harmony between initial conditions of pavement, specific treatment applied on the pavement, and the expected pavement conditions after improvement. The experimental adjustment consists of four analysis steps: (1) randomly range IRI value from $0-7 \mathrm{~m} / \mathrm{km}$ with an incremental of $0.1 \mathrm{~m} / \mathrm{km}$; (2) calculate the pavement performance jump corresponding to each IRI value and treatment type; (3) computationally trial to get the possible range of IRI for each descriptive performance rating; (4) test the compatibility of pre-and post-pavement conditions in respect to the applied treatment type. Steps 3 and 4 will be repeated until the appropriate ranges of IRI that perfectly fit the rehabilitation treatments for all pavement condition stages are generated. The final result is validated with the pavement condition criteria stated in Shafizadeh et al. (2002). The validation confirms an agreement of final computational ranges and the highway practice in identifying the correlation between the subjective pavement performance rating and the expected IRI value.

The second challenge (see Box 2 in Figure 4-2), which is related to the differences in defining the types of treatments among the literature, can be solved by integrative and comparative approaches. First, the pavement condition rating (PCR) threshold ranges for the network-level corrective action category defined in ODOT (1999) are combined with the condition rating thresholds in INDOT (2001)(as cited in Khurshid 2010). Since the treatment actions for different levels of IRI are needed and the ODOT's report (1999) only provides the relation between the PCR value and the action category, the INDOT's study 
(2001) is integrated to find the correlation between PCR and IRI. Table 4-1 shows the modified result based on the integration of these two literature works.

Table 4-1 Correlations between PCR, IRI, and action category from the past literature

\begin{tabular}{|c|c|c|}
\hline PCR & IRI & Action Category \\
\hline $\mathrm{PCR}>85$ & $\mathrm{IRI}<1.97(125)$ & No action required \\
$85>\mathrm{PCR}>75$ & $1.97(125)<\mathrm{IRI}<2.76(175)$ & Preventive Maintenance \\
$75>\mathrm{PCR}>55$ & $2.76(175)<\mathrm{IRI}<4.34(275)$ & Minor Rehabilitation \\
$\mathrm{PCR}<55$ & $\mathrm{IRI}>4.34(275)$ & Major Rehabilitation \\
\hline
\end{tabular}

Considering the IRI value for each treatment category in Table 4-1, the comparative analysis is performed to observe an agreement of the treatment's definition mentioned in de la Garza and Krueger (2007) with the action category. Table 4-2 presents the comparative result given from the aforementioned analysis process.

Table 4-2 Comparative result in de la Garza and Krueger (2007)

\begin{tabular}{|c|c|}
\hline $\begin{array}{c}\text { Treatment activities } \\
\text { (from de la Garza and } \\
\text { Krueger (2007)) }\end{array}$ & $\begin{array}{c}\text { Action Category } \\
\text { (from table 1) }\end{array}$ \\
\hline Thin overlay & Preventive Maintenance \\
Thick overlay & Minor Rehabilitation \\
Rehabilitation & Major Rehabilitation \\
Reconstruction & N/A \\
\hline
\end{tabular}

The previous comparative analysis creates the categorization of treatment activities identified in de la Garza and Krueger (2007). However, the compatibility between the types of treatments in Irfan (2010) and de la Garza and Krueger (2007) is still missing. The treatment identified in Irfan's study (2010) is therefore categorized in respect to the action 
category in Table 4-1 for further comparative analysis. The result from this comparison can be illustrated in Table 4-3.

Table 4-3 Lists of treatment activities from Irfan (2010) for each action category

\begin{tabular}{|c|c|}
\hline Action Category & $\begin{array}{c}\text { Treatment activities } \\
\text { (from Irfan (2010)) }\end{array}$ \\
\hline Preventive Maintenance & $\begin{array}{c}\text { Thin HMA overlay } \\
\text { Micro-surfacing }\end{array}$ \\
\hline Minor Rehabilitation & HMA overlay functional \\
\hline Major Rehabilitation & $\begin{array}{c}\text { HMA overlay structural } \\
\text { Partial 3R standards }\end{array}$ \\
\hline
\end{tabular}

According to Table 4-3, there are several treatment activities that can be applied for preventive maintenance and major rehabilitation. However, there are specific conditions for implementing some types of treatments. For example, the Partial 3R Standards covers several detailed tasks other than the structural repair, such as shoulder widening and removal or protection of roadside obstacles (Irfan 2010). In this study, the past literature is accessed to select only widely-used treatments for the analysis. Thin HMA overlay is selected as the preventive maintenance action because of its popularity among highway maintenance and rehabilitation activities (Irfan 2010). In addition, structural HMA overlay is adopted for the major rehabilitation category since it is the most commonly-implemented for paved roads in the U.S. (Tucson 2012). From this step, the lists of treatments from de la Garza and Krueger (2007) will be comparably replaced by the selected treatments from Irfan (2010). Table 4-4 demonstrates an equivalence in the treatment activities from the past literature. 
Table 4-4 Lists of treatment activities from Irfan (2010) for each action category

\begin{tabular}{|c|c|}
\hline $\begin{array}{c}\text { Treatment activities } \\
\text { (from de la Garza and } \\
\text { Krueger (2007)) }\end{array}$ & $\begin{array}{c}\text { Treatment activities } \\
\text { (from Irfan (2010)) }\end{array}$ \\
\hline Thin overlay & Thin HMA overlay \\
Thick overlay & HMA overlay functional \\
Rehabilitation & HMA overlay structural \\
\hline
\end{tabular}

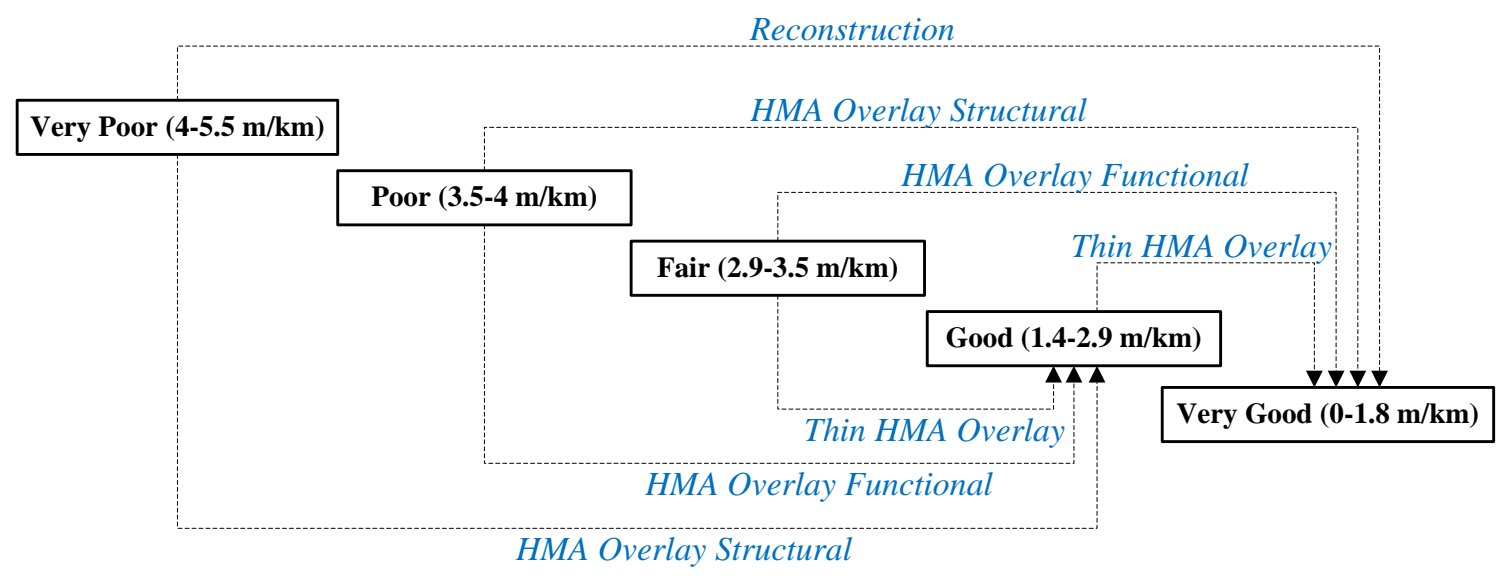

Figure 4-3 Modification of possible treatments for pavement condition stages

The final result from all analysis steps can be presented in Figure 4-3, which states the range of performance index associated with all five levels of performance rating. The algorithm in this figure will be used for identifying the rehabilitation treatment alternatives of the aging transportation network in the example of this paper. It is worth noting that the pavement with an IRI larger than $5.5 \mathrm{~m} / \mathrm{km}$ is always considered to need to be reconstructed in this study because the reconstruction is the only feasible method for improving pavement conditions when the pavement is badly deteriorated with significant structural damages (Carnahan et al. 1987).

Assigning highway rehabilitation activity to any deteriorating pavement contributes to the improvement of pavement conditions (Chootinan et al. 2006; Giustozzi et al. 2012; 
Irfan et al. 2008). The IRI index is therefore expected to decrease after applying the rehabilitation treatment (Lidicker et al. 2012). The difference of IRI before- and aftertreatment applications can be defined as an effectiveness representing the level of treatment's capability in improving the performance of a deteriorating roadway pavement. Treatment effectiveness could be measured in terms of an extended service life, performance jump, and post-treatment performance trend (Irfan et al. 2008; Lamptey et al. 2008; Wang et al. 2003). As already known, as rehabilitation helps resetting or recovering the deterioration process of a highway pavement (Irfan 2010), different treatments will affect the pavement performance differently (Chootinan et al. 2006). This confirms the significance of highway treatment selection in pavement deterioration and therefore energy consumption (Zhang et al. 2012).

\subsection{Network Pavement Performance Evaluating Module}

This section explains how performance conditions of all pavements throughout the network as a result of rehabilitation treatment application can be evaluated. It subjects to predict the future conditions of the pavement after receiving the rehabilitation treatment. As the selection of treatment has a significant impact on the improvement of pavement conditions after treatment, two main indicators commonly used in the past literature for measuring the treatment effectiveness - performance jump and post-treatment performance trend - are included in this section. Accordingly, this section is designed to start with the introductions of performance jump and post-treatment performance trend and their relevant literature applied in this research study. Afterwards, the network pavement performance evaluating module that is capable of facilitating a calculation of network pavement 
performance along an analysis period is introduced. The explanations and calculation algorithms associated to the developed module is also provided as follows.

\subsubsection{Pavement Performance Abrupt Improvement}

Abrupt performance improvement in pavement resulting from the rehabilitation treatment application is often referred to as the performance jump. It is defined as a sudden or immediate increase in pavement performance upon the implementation of the treatment, in which it is measured as the difference in pavement conditions at just before and right after receiving the treatment. Performance jump can be determined with a constant number representing an average value of IRI drop in pavement or a function in terms of pavement and treatment attributes (Irfan et al. 2008), which can be generalized as shown in Equation 4-1 (Geoffroy 1996; Irfan et al. 2009).

$$
\mathrm{PJ}=\mathrm{f}(\tilde{x})
$$

Where PJ = performance jump of a treatment at the year of application (in $/ \mathrm{mile}$ or $\mathrm{m} / \mathrm{km}$ ); $\mathrm{f}(\tilde{x})=\mathrm{a}$ function of explanatory variables, such as pre-treatment performance and treatment type.

In this study, the performance jump models from Irfan (2010) are adopted for the model application. The mathematical form for calculating the performance jump used in this past study was given in Equation 4-2.

$$
\mathrm{PJ}_{\mathrm{s}}=\mu(1)_{\mathrm{s}}+\mu(2)_{\mathrm{s}} *\left[\operatorname{lnPI} \mathrm{I}_{\text {trig }}\right]
$$

Where $\mathrm{PJ}_{\mathrm{s}}=$ performance jump at the time of application for treatment option (s); $\mathrm{PI}_{\text {trig }}=$ pavement performance trigger value for treatment (s) at the time of application; $\mu(1)=$ 
constant value corresponding to the treatment option (s); and $\mu(2)=$ treatment-specific parameter for treatment (s).

It is worth noting that the values of parameters, $\mu(1)$ and $\mu(2)$ are varied depending on the functional class of the pavement road. For a simple calculation of the case study that will be mentioned later in this paper, all parameter values are averaged between two functional classes - interstate (IS) pavements and non-interstate national highway (NHSNon IS) pavements, as these two classes can be good representatives of the roads in the application example of this paper. Table 4-5 represents the modified values of parameters $\mu(1)$ and $\mu(2)$ corresponding to each treatment option.

Table 4-5 Modified parameters for performance jump models (modified from Irfan 2010)

\begin{tabular}{|l|c|c|}
\hline \multicolumn{1}{|c|}{ Treatment Type } & $\boldsymbol{\mu ( 1 )}$ & $\boldsymbol{\mu ( 2 )}$ \\
\hline Thin HMA overlay & -272.458 & 70.412 \\
\hline HMA overlay functional & -285.723 & 73.673 \\
\hline HMA overlay structural & -326.194 & 83.889 \\
\hline
\end{tabular}

\subsubsection{Post-Treatment Pavement Performance}

This section is related to the performance of a treated pavement after receiving rehabilitation. Indeed, post-treatment performance can be defined as the deterioration trend of a pavement at any given year within a treatment service life or considering time, as a consequence of treatment application. Therefore, the trend can be expressed as the IRIincreasing curve over a considering time until a next intervention is performed. This increase in IRI is the effect of deterioration due to several functional attributes, for example the accumulated traffic loading and the age of pavement. The general form for the post- 
treatment pavement performance trend can be expressed as Equation 4-3 (Geoffroy 1996; Irfan et al. 2009).

$$
\mathrm{PI}=\mathrm{f}(\tilde{\varphi}, t)
$$

Where PI = pavement performance indicator (IRI in this study) (in $/ \mathrm{mile}$ or $\mathrm{m} / \mathrm{km}$ ) for an implemented pavement at a time of application (t); $\tilde{\varphi}=$ explanatory variables, such as treatment type and average annual truck traffic volume; $\mathrm{t}=$ time or treatment service life (years).

As aforementioned, a selection of treatment application significantly affects the conditions of the pavement over the service life. In addition to the short-term impact (performance jump), the literature reveals the impact of different treatment options on the long-term pavement performance (post-treatment trend). Similar to performance jump, this paper adopts the post-treatment pavement performance trend models from Irfan (2010) for the case study in the application example section, in which the functional form for calculating the post-treatment performance can be generalized, as shown in Equation 4-4.

$$
\mathrm{PI}=e^{(\alpha+\beta . A A T A . t+\gamma \cdot A N D X . t)}
$$

Where PI = pavement performance indicator (IRI in this study) (in/mile or $\mathrm{m} / \mathrm{km})$; $\mathrm{t}=$ treatment service life (in years); AATA = average annual truck traffic volume (in millions per year); ANDX = average annual freeze index (in thousands per years); $\alpha=$ constant value corresponding to the treatment option; and $\beta$ and $\gamma=$ specific coefficients for model explanatory variables.

The values of parameters $\alpha, \beta$ and $\gamma$ are also averaged between the interstate (IS) pavements and non-interstate national highway (NHS-Non IS) based on the same reason 
previously mentioned in the performance jump section. Table 4-6 then demonstrates the modified values of the parameters that will be used for the post-treatment performance calculation.

Table 4-6 Modified parameters for post-treatment performance models (modified from Irfan 2010)

\begin{tabular}{|l|c|c|c|}
\hline \multicolumn{1}{|c|}{ Treatment Type } & $\boldsymbol{\alpha}$ & $\boldsymbol{\beta}$ & $\boldsymbol{\gamma}$ \\
\hline Thin HMA overlay & 4.243 & 0.027 & 0.056 \\
\hline HMA overlay functional & 4.131 & 0.018 & 0.075 \\
\hline HMA overlay structural & 3.971 & 0.022 & 0.142 \\
\hline New full-depth HMA construction & 4.023 & 0.081 & 0.028 \\
\hline
\end{tabular}

\subsubsection{Evaluating Network Pavement Performance}

This section proposes an algorithm for calculating the long-term pavement conditions, in which the effects of pavement jump and post-treatment pavement performance from the previous two subsections are combined. In this paper, long-term pavement conditions are defined as the level of pavement conditions that will be varied by depending on decisions in rehabilitating pavement over a predetermined analysis period, which contains repetitive cycles of rehabilitation programs. The algorithm herein takes into account the effect of rehabilitation decisions in every program within a planning period.

The algorithm is developed by assuming that decisions in rehabilitation programs are completely made by transportation agencies before rehabilitation activities start (at year 0 of each rehabilitation program. Figure 4-4 demonstrates a seven-step process with a detailed explanation of each step as follows: 


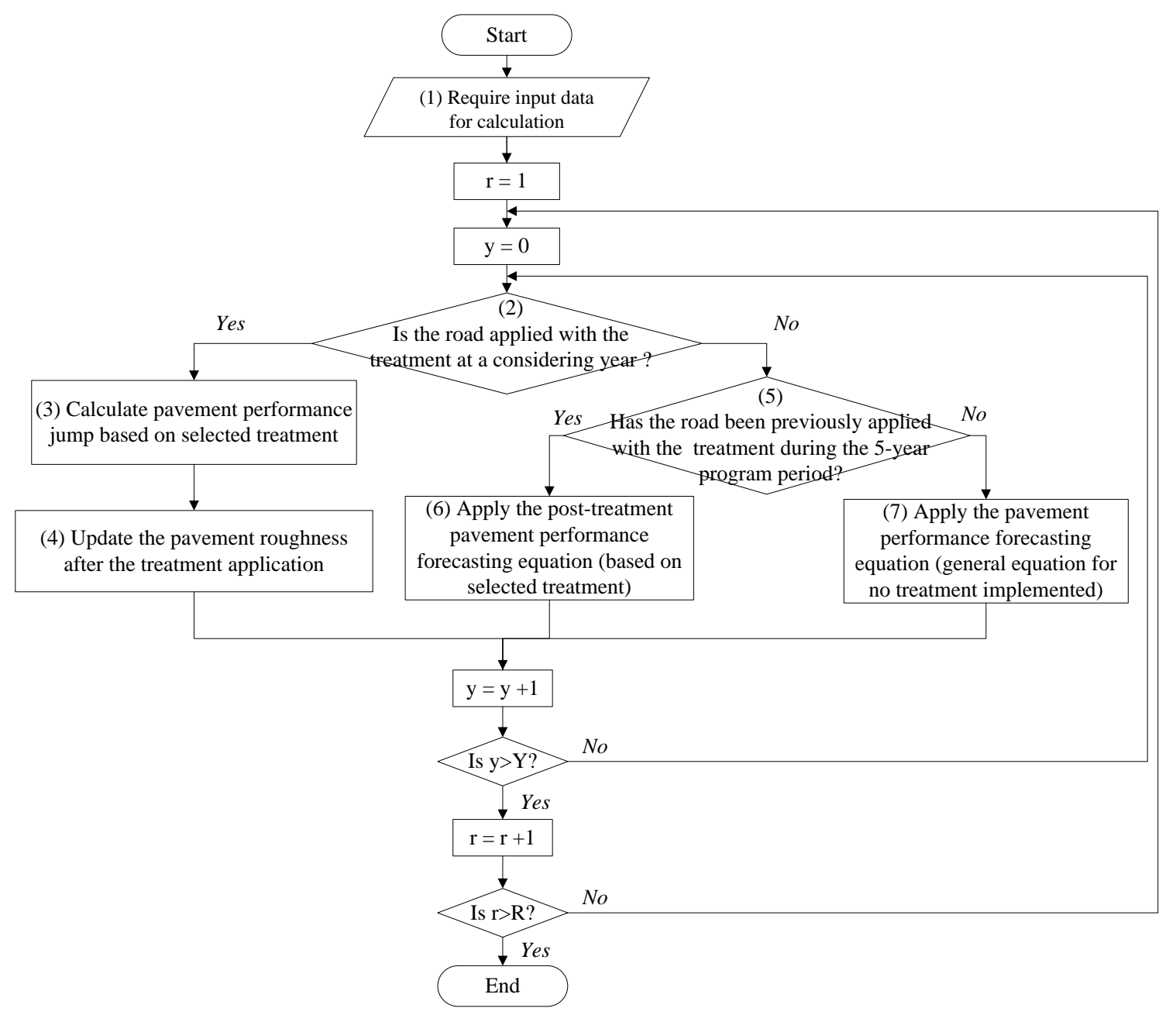

Figure 4-4 Calculation algorithm for long-term pavement conditions evaluation

(1) Collect all input required for the calculation, including the selected rehabilitation treatments determined by transportation planners, and annual truck traffic volume, the climate effect factor, and current pavement conditions (IRI) that can be retrieved from transportation database.

(2) Check whether a road is going to be applied with a treatment at a considering year. If yes, proceed to step 3; otherwise go to step 5 . 
(3) Calculate the performance jump for a rehabilitated road section (r) by using the pavement performance jump model corresponding to a treatment type selected by transportation planners.

(4) Update the pavement conditions (IRI) for a rehabilitated road section (r) after the treatment application by taking into account the effects of performance jump. In this step, the effect of IRI drop due to the improvement of pavement performance is considered. The general form of this calculation can be expressed as Equation 4-5. Then, proceed to year $\mathrm{y}+1$.

$$
I R I_{\text {post }}^{r}=I R I_{\text {pre }}^{r}-P J_{s}^{r}
$$

Where IRI ${ }_{\text {pre }}^{\mathrm{r}}, \mathrm{IRI}_{\text {post }}^{\mathrm{r}}=$ IRI value on road section ( $\mathrm{r}$ ) before and after an application of treatment option (s), respectively; and $\mathrm{P} \mathrm{s}_{\mathrm{s}}^{\mathrm{r}}=$ performance jump at the time of application for treatment option

(5) Check whether a road section (r) is previously applied with a rehabilitation treatment. If yes, go to step 6; otherwise proceed to step 7.

(6) Apply the post-treatment performance model developed in the past studies to calculate the long-term pavement conditions for a previously-treated road section, based on a specific treatment type applied earlier. Then, proceed to year $\mathrm{y}+1$.

(7) Apply the pavement performance forecasting equation from the past literature to predict the long-term pavement performance in terms of IRI for a non-previously-treated road section. Then, proceed to year $\mathrm{y}+1$.

It should be noted that the calculation from steps 2-7 are repeated for all road sections ( $\mathrm{r}=1$ to $\mathrm{R}$ ) in the transportation network. 
As mentioned in the previous sections, the IRI will abruptly drop when applying a treatment. In the pavement conditions curve, this drop can be represented with a vertical decreasing line as shown in Figure 4-5. Subsequently, the IRI tends to increase over the year due to deterioration from the pavement use. To this end, the variations in IRI values cause the changes in vehicle fuel usage (Yu and Lu 2012; Zaabar and Chatti 2011; Zhang et al. 2009) and then total energy consumption at the network level.

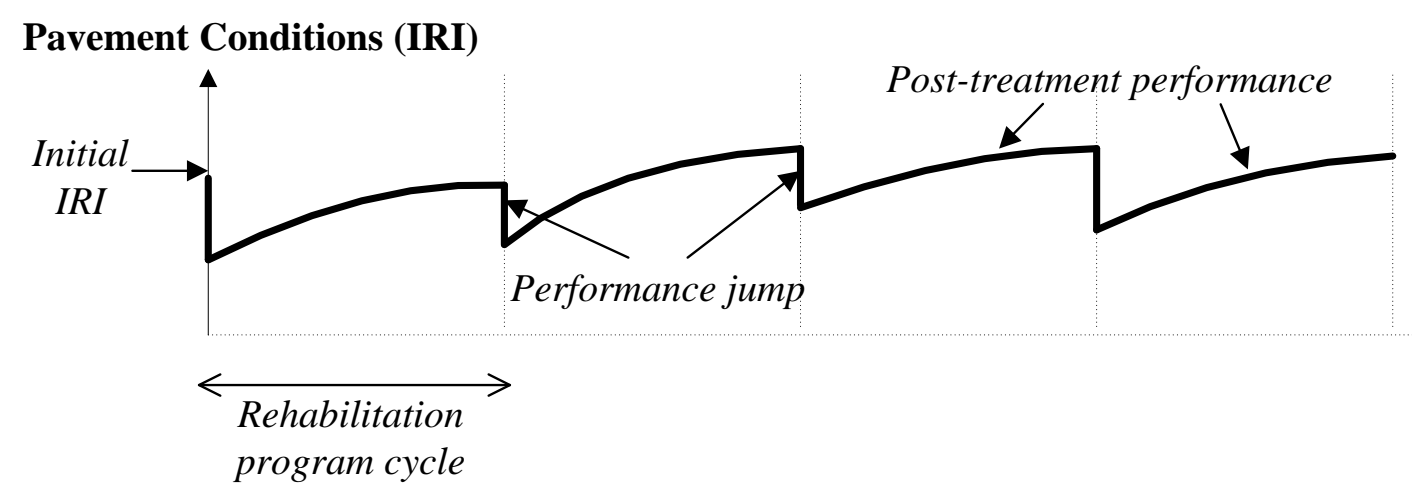

Figure 4-5 Pavement conditions curve represents the effects of rehabilitation efforts

\subsection{Network Energy Consumption Estimating Module}

The objective of this module is to estimate total energy consumption of transportation networks resulting from the implementation of rehabilitation efforts. In order to achieve this objective, energy consumed in transportation networks is grouped into two main categories: (1) energy consumed during highway construction operations; and (2) energy consumed during regular operation after the completion of highway rehabilitation works to improve pavement conditions.

First, network energy consumption during highway construction is expected to increase due to the reduction in vehicle speed when travelling through construction zones. This reduction in vehicle speed can cause an increase in the fuel consumption rate (Chatti 
and Zaabar 2012). Moreover, the vehicle speed reduction can affect traffic using the road under rehabilitation to change due to some travelers opting to use alternative routes. The total change in fuel consumption during the highway construction operations will depend on the number of road sections in the network undergoing rehabilitation, road section lengths, and duration of construction operations.

Second, the improvement in pavement conditions, as a result of the rehabilitation efforts, will also affect changes to the network energy consumption. In this module, the pavement roughness index (IRI) is used to represent pavement conditions. The IRI of road segments that undergo rehabilitation will decrease after rehabilitation and will therefore cause a significant reduction in energy consumption (Amos 2006), compared to prerehabilitation. This type of energy consumption is calculated as lifecycle energy consumption for the transportation network over an analysis span of $\mathrm{Y}$ years after rehabilitation. This lifecycle energy consumption takes into consideration the gradual increase over time in IRI and therefore energy consumption. Network energy consumption is expected to be the lowest directly after rehabilitation and then gradually increases with time until the network is due for new rehabilitation, as shown in Figure 4-6.

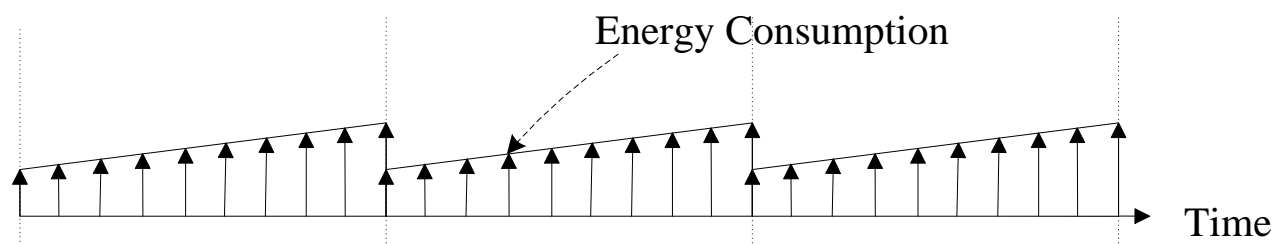

$1^{\text {st }}$ Rehabilitation $\quad 2^{\text {nd }}$ Rehabilitation $\quad 3^{\text {rd }}$ Rehabilitation $\quad 4^{\text {th }}$ Rehabilitation

Figure 4-6 Impact of rehabilitation efforts on energy consumption 


\section{$\underline{\text { Model Development }}$}

The flowchart in Figure 4-7 shows a six-step process for calculating total energy consumption for the entire transportation network, as follows:

(1) Collect a necessary input data for the next-step calculation. The parameters are categorized into two main groups corresponding to the phase of operation, during the construction and post-rehabilitation. The during-construction calculation requires the following inputs - total number of road sections in the networks, current pavement conditions of each road section, length of the road section, construction duration, and vehicle speed and traffic volume at work-zone conditions. Similarly, the necessary inputs for the post-rehabilitation operation are total number of road sections in the networks, number of years in lifecycle period, pavement conditions at year (y) after rehabilitation, length of the road section, and traffic volume and vehicle speed at free-flow conditions on each road section.

(2) Estimate the fuel consumption rate under work-zone conditions by using the equations in Table 3-1 for calculating the vehicle fuel consumption rate that is applicable to the transportation network implementation.

(3) Calculate the expected network energy consumption caused from the construction operations. This expected energy consumption represents fuel consumption of all vehicles traveling on the road during construction, before the operation is resumed (See Equation 4-6). Steps 2 and 3 are repeated for all road sections ( $r=1$ to $R$ ) to obtain the total energy consumption for the entire network. 


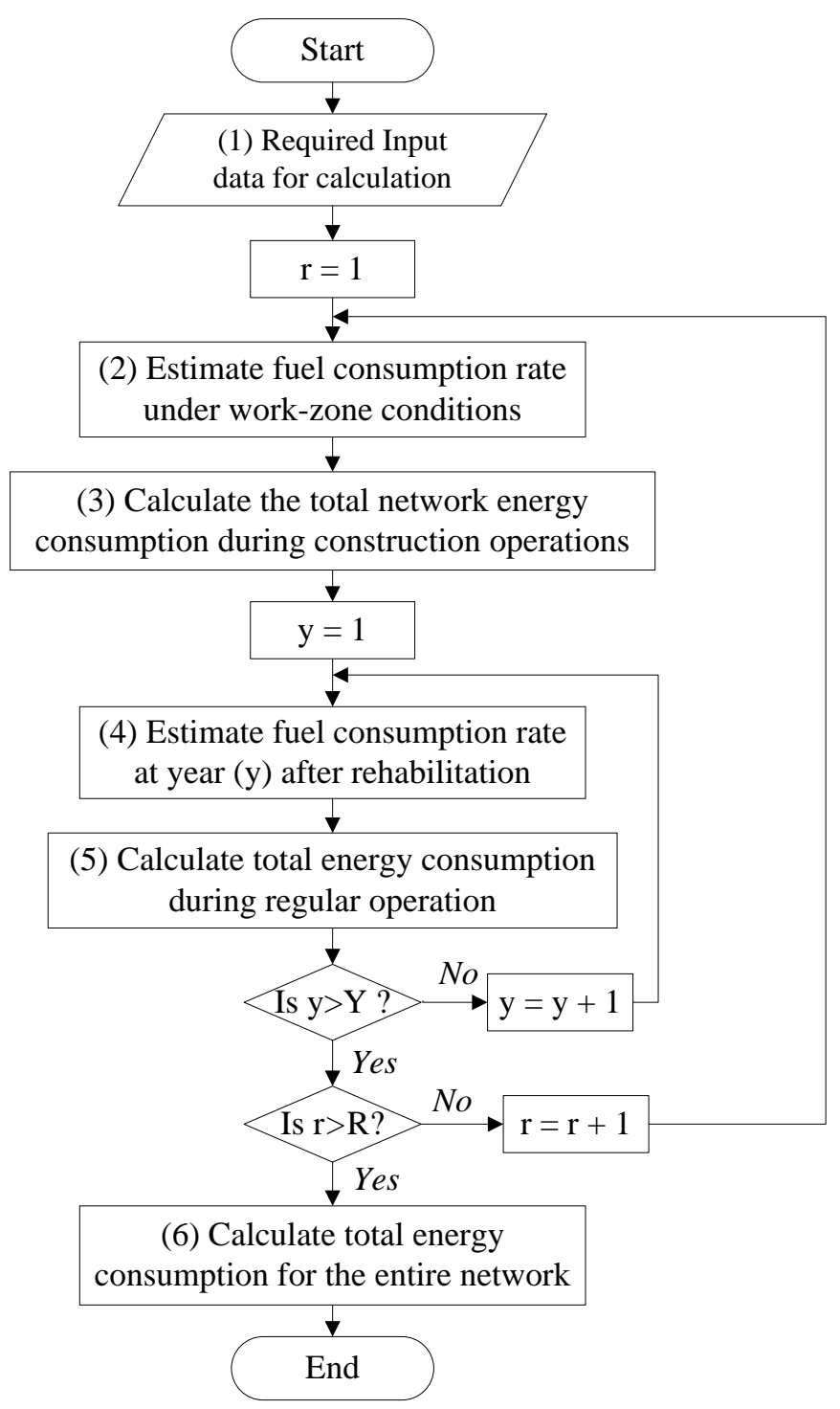

Figure 4-7 Calculation process of transportation network energy consumption

$$
\mathrm{TFC}=\sum_{\mathrm{r}=1}^{\mathrm{R}} \mathrm{V}_{\mathrm{W}, \mathrm{r}} * \mathrm{~L}_{\mathrm{r}} * \mathrm{D}_{\mathrm{r}} * \mathrm{FR}_{\mathrm{W}, \mathrm{r}}
$$

Where, TFC $=$ total network fuel due to construction operations; $\mathrm{R}=$ number of road sections in the network; $\mathrm{V}_{\mathrm{W}, \mathrm{r}}=$ traffic volume under work-zone conditions; $\mathrm{L}_{\mathrm{r}}=$ length of road section $(\mathrm{r}) ; \mathrm{D}_{\mathrm{r}}=$ construction duration that affects road section $(\mathrm{r})$; and $\mathrm{FR}_{\mathrm{W}, \mathrm{r}}=$ fuel consumption rate under work-zone conditions (from step 2). 
(4) Estimate the vehicle fuel consumption rate under the post-rehabilitation stage. Similar to step 2, the fuel consumption rate can be estimated based on the developed equations in Table 3-1. This rate is calculated yearly over an analysis of y years after rehabilitation.

(5) Calculate total energy consumption during regular operation by using Equation 4-7. Steps 4 and 5 are repeated over a predetermined lifecycle span ( $y=1$ to $\mathrm{Y})$ and all road sections ( $r=1$ to $R$ ) to attain total network energy consumption during the postrehabilitation operation.

$$
\mathrm{TF}=\sum_{\mathrm{y}=1}^{\mathrm{Y}} \sum_{\mathrm{r}=1}^{\mathrm{R}} \mathrm{V}_{\mathrm{r}} * \mathrm{~L}_{\mathrm{r}} * \mathrm{FRN}_{\mathrm{r}}^{\mathrm{y}}
$$

Where, $\mathrm{TF}=$ total fuel consumption during regular operation; $\mathrm{Y}=$ number of years to new rehabilitation effort; $\mathrm{R}$ = number of road sections in the network; $\mathrm{V}_{\mathrm{r}}=$ traffic volume (in terms of AADT) on road section (r); $\mathrm{L}_{\mathrm{r}}=$ length of road section (r); and $\mathrm{FRN}_{\mathrm{r}}{ }^{\mathrm{y}}=$ fuel consumption rate of road project (r) after year (y) of rehabilitation (from step 4).

(6) Calculate total energy consumption for the entire network by summing the during-construction and post-rehabilitation energy consumption estimated in steps 3 and 5.

\subsection{Public Cost Estimating Module}

The main objective of this module is to evaluate and model the public cost from implementing the highway rehabilitation effort on transportation networks. In this study, the public cost is measured in terms of the travel-delay cost increased from the expected traffic delay that travelers experience during the construction operations. This type of cost is one component in user costs, and it is usually considered as the most significant impact on road users. In addition to the impacts on the road undergoing repair, rehabilitation 
affects traffic conditions on the other roads in the network. For instance, travelers are forced to take longer detours with the work-zone speed limit. Additionally, some travelers are tempted to choose faster routes in order to avoid traffic disruption that occurs from the construction activities. All traffic diversions can increase traffic volume on the alternated routes and eventually the road capacity may be exhausted. Accordingly, all vehicles including the routine travelers traveling on the road will be affected from traffic congestion and a significantly reduced traveling speed. This means rehabilitation efforts are anticipated to alter the network traffic patterns, and therefore increase total travel time in the transportation network.

Generally speaking, modeling traffic diversion on transportation networks is very challenging due to dynamic preferences of travelers in selecting the driving routes and indeterministic changes of traffic demand through networks. As a result, to maintain simplicity, the public cost in this study is modeled only based on the impact of speed reduction on the constructing roads. Therefore, the cost of travel delays can be estimated from traffic volume, length of road, change in travel time, and unit time value. The traveldelay time can be measured in hours per one vehicle (hr/veh). The total cost of travel delays will be calculated with the multiplication of the entire travel-delay time by the unit time value $(\$ / h r)$.

\section{Model Development}

Figure 4-8 illustrates a five-step procedure for estimating total public cost resulting from implementing the rehabilitation efforts, as follows:

(1) Gather all input data required for the calculation. These inputs have to be predetermined by transportation planners, and most of them can be accessed from the 
rehabilitation plan or the transportation agencies' database. This required data consists of (1) number of road sections in the network; (2) length of the road section; (3) construction duration that affects road section; (4) vehicle speed under free-flow conditions; (5) vehicle speed under work-zone conditions; and (6) unit time value (\$/one unit of time).

(2) Estimate the travel time of each road section (r) under free-flow conditions by dividing its length by the vehicle free-flow speed on the road, as shown in Equation 4-8.

$$
\mathrm{T}_{\mathrm{F}, \mathrm{r}}=\frac{\mathrm{L}_{\mathrm{r}}}{\mathrm{S}_{\mathrm{F}, \mathrm{r}}}
$$

Where, $\mathrm{T}_{\mathrm{F}, \mathrm{r}}=$ travel time under free-flow conditions; $\mathrm{L}_{\mathrm{r}}=$ length of the road section; and $\mathrm{S}_{\mathrm{F}, \mathrm{r}}=$ average vehicle speed under free-flow conditions.

(3) Estimate the travel time of each road section in the network under work-zone conditions. A similar equation to step 2 can be adopted for the calculation under the construction operations, as shown in Equation 4-9.

$$
\mathrm{T}_{\mathrm{W}, \mathrm{r}}=\frac{\mathrm{L}_{\mathrm{r}}}{\mathrm{S}_{\mathrm{W}, \mathrm{r}}}
$$

Where, $\mathrm{T}_{\mathrm{W}, \mathrm{r}}=$ travel time under work-zone conditions; $\mathrm{L}_{\mathrm{r}}=$ length of the road section; and $\mathrm{S}_{\mathrm{W}, \mathrm{r}}=$ average vehicle speed under work-zone conditions.

(4) Calculate the change in travel time or travel delay $\left(\Delta \mathrm{T}_{\mathrm{r}}\right)$ due to the rehabilitation by subtracting travel time under free-flow from work-zone conditions. Steps 2 to 4 are repeated for all road sections ( $r=1$ to $R$ ) to attain the travel delay for each road section throughout the network. 


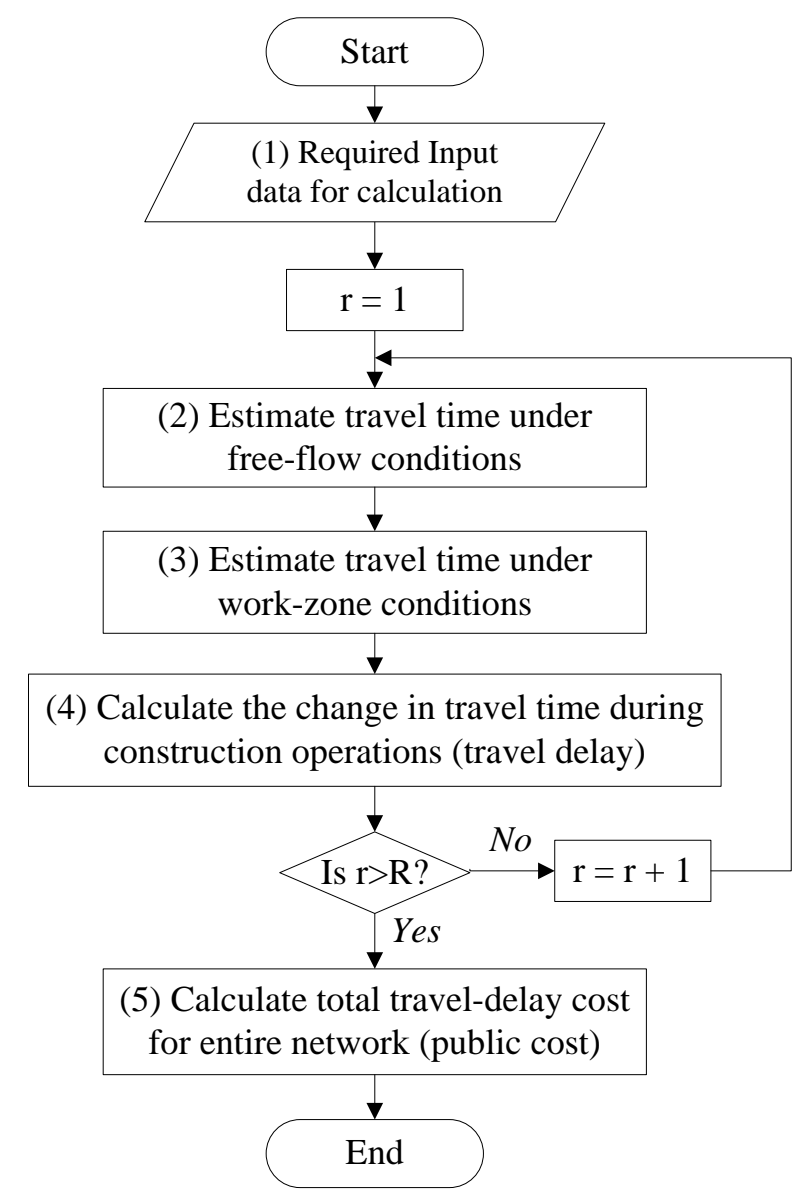

Figure 4-8 Calculation procedure of expected public cost on transportation network

(5) Calculate public cost, in terms of travel-delay cost herein, for the entire network. Equation 4-10 represents the estimation of entire travel-delay cost during the construction. The cost of travel delays can be estimated from the change in traffic volume, length of the road section, change in travel time, and unit time value. The last parameter, the predefined value from the user, will convert total travel time (hr) to monetary value $(\$ / \mathrm{hr})$.

$$
\mathrm{TTC}=\mathrm{UT} * \sum_{\mathrm{r}=1}^{\mathrm{R}}\left[\left(\mathrm{V}_{\mathrm{W}, \mathrm{r}} * \mathrm{~T}_{\mathrm{W}, \mathrm{r}}\right)-\left(\mathrm{V}_{\mathrm{F}, \mathrm{r}} * \mathrm{~T}_{\mathrm{F}, \mathrm{r}}\right)\right] * \mathrm{D}_{\mathrm{r}}
$$


Where, TTC = total travel-delay cost during construction operations; UT = unit time value; $\mathrm{R}=$ number of road sections in the network; $\mathrm{V}_{\mathrm{W}, \mathrm{r}}=$ traffic volume on road section $(\mathrm{r})$ under work-zone conditions; $\mathrm{V}_{\mathrm{F}, \mathrm{r}}=$ traffic volume on road section (r) under free-flow conditions; and $\mathrm{D}_{\mathrm{r}}=$ construction duration that affects road section ( $\mathrm{r}$ ).

\subsection{Public Benefits Estimating Module}

The main objective of this module is to evaluate the expected public benefits from the implementation of the rehabilitation program. This model takes into consideration the impacts of the rehabilitation effort that happens during the regular operation in the public point of view. The public benefits are typically measured as cost savings in traveling on the network. These savings can be less travel time between origin and destination, and a decrease in vehicle operating costs as a result from the improvement in pavement conditions after rehabilitation. However, only the savings in vehicle operating costs are the focuses of this study, since the pavement's surface smoothness has very limited impact on vehicle operating speed and driving behavior (Wang 2013; Zaniewski et al. 1982). This means the travel time is not significantly changed after rehabilitation compared to the prerehabilitation stage. Therefore, the impact of travel time reduction is negligible from this study.

According to Bennett and Greenwood (2003), vehicle operating costs are the costs related to fuel consumption, tire depreciation, repair and maintenance, vehicle depreciation, and license and insurance. However, the first three main components are commonly considered in the existing models (Chatti and Zaabar 2012). In this study, the savings in fuel consumption, tire depreciation and repair and maintenance costs, which are affected by pavement conditions, are included for estimating the public benefits. 
Fuel consumption cost is the cost of total fuel that vehicle consumes while traveling on a road. This type of cost can be calculated based on total number of vehicles on the road, length of the road section, total number of lanes on the road, and the vehicle fuel consumption rate. The savings in fuel consumption cost can be resulted from the cost difference at before- and after- rehabilitation treatment.

Tire depreciation cost is the cost resulting from tire tread weariness. Normal vehicle usage makes the tread gradually shallower and decreases overall tire performance. Although it has gained less attention in comparison to fuel consumption, tire depreciation is one of the important components in vehicle operating costs (Chatti and Zaabar 2012). The savings in tire depreciation cost accumulated on each road section can be calculated based on the number of all vehicles traveling through the road section, length of the road section, and variation in the tire depreciation cost rate. Rehabilitation activities will help improving the conditions of the road pavement. This improvement leads to the savings in tire depreciation cost by comparing the pre- and post-rehabilitation conditions over a lifecycle span.

Repair and maintenance costs consist of two main components, which are vehicle part consumption and associated labor costs (Chatti and Zaabar 2012). Similar to fuel consumption and tire depreciation cost, traffic volume, length of the road section, and the repair and maintenance costs rate directly affect the total costs for the entire network. Also, the savings from rehabilitation implementation on the transportation network can be calculated from the difference between the repair and maintenance costs before and after rehabilitation. 


\section{$\underline{\text { Model Development }}$}

To estimate total public benefits of transportation network rehabilitation, the following ten steps are used, as shown in Figure 4-9.

(1) Collect input parameters required for the estimation. Some parameters tend to be available in the pavement management system database (i.e. pavement conditions at the pre-rehabilitation stage, average traveling speed at regular operation, traffic volume, and length of road section). However, some inputs are determined based on the decisions of transportation planners or the rehabilitation plan (i.e. number of lifecycle year, number of road sections under rehabilitation, and pavement conditions at year (y) after rehabilitation).

(2) Estimate the fuel consumption cost rate at the pre-rehabilitation stage by using equations in Table 3-1, which are best-suited for the transportation network application.

(3) Estimate the fuel consumption rate at year (y) after rehabilitation over the predefined analysis period. The equations from Table 3-1 will be also used for the calculation in this step.

(4) Calculate the rate of fuel consumption cost savings at year (y) over an analysis lifespan. The results from steps 2 and 3 are entered in Equation 4-11 to determine the cost savings rate. The calculation is repeated from steps 2 to 4 for each road section and each year of rehabilitation.

$$
\Delta \mathrm{FR}_{\mathrm{r}}^{\mathrm{y}}=\mathrm{FRI}_{\mathrm{r}}^{\mathrm{y}}-\mathrm{FRN}_{\mathrm{r}}^{\mathrm{y}}
$$

Where, $\Delta \mathrm{FR}^{\mathrm{y}} \mathrm{r}=$ rate of fuel consumption savings of road section $(\mathrm{r})$ at year $(\mathrm{y})$ after rehabilitation; $\mathrm{FRI}_{\mathrm{r}} \mathrm{r}_{\mathrm{r}}$ fuel consumption rate of road section (r) at pre-rehabilitation conditions; and $\mathrm{FRN}^{\mathrm{y}} \mathrm{r}_{\mathrm{r}}=$ fuel consumption rate of road section (r) after year (y) of rehabilitation. 
(5) Estimate the tire depreciation cost rate at the pre-rehabilitation stage. The mathematical equations developed as shown in Table 3-2 will be applied for estimating the cost rate in this step.

(6) Estimate the tire depreciation cost rate at year (y) after rehabilitation over the predefined lifecycle span. A similar equation used in step 5 will be also adopted for the calculation in this step.

(7) Calculate the rate of tire depreciation savings at year (y) after rehabilitation. The saving rate can be calculated by using the results from steps 5 and 6 , as shown in Equation 4-12. Steps 5 to 7 are repeated for all road sections undergoing rehabilitation ( $\mathrm{r}=$ 1 to $\mathrm{R}$ ) to obtain the rate of tire depreciation savings at year (y) for each road section in the network.

$$
\Delta \mathrm{DR}_{\mathrm{r}}^{\mathrm{y}}=\mathrm{DRI}_{\mathrm{r}}^{\mathrm{y}}-\mathrm{DRN}_{\mathrm{r}}^{\mathrm{y}}
$$

Where, $\Delta \mathrm{DR}_{\mathrm{r}}^{\mathrm{y}}=$ rate of tire depreciation savings of road section $(\mathrm{r})$ at year $(\mathrm{y})$ after rehabilitation; $\mathrm{DRI}_{\mathrm{r}}^{\mathrm{y}}=$ tire deprecation rate of road section $(\mathrm{r})$ at pre-rehabilitation conditions; and $\mathrm{DRN}_{\mathrm{r}}^{\mathrm{y}}=$ tire depreciation rate of road section $(\mathrm{r})$ after year $(\mathrm{y})$ of rehabilitation.

(8) Estimate the rate of repair and maintenance costs at the pre-rehabilitation stage. Similar to the other types of costs, the network-application equations in Table 3-3 will be used for the calculation. 


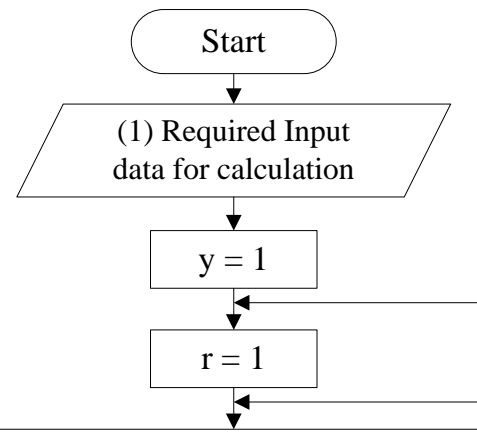

(2) Estimate fuel consumption cost rate at pre-rehabilitation stage

(3) Estimate fuel consumption cost rate at year y after rehabilitation

(4) Calculate the rate of fuel consumption savings at year y after rehabilitation

(5) Estimate tire depreciation cost rate at pre-rehabilitation stage

(6) Estimate tire depreciation cost rate at year y after rehabilitation

(7) Calculate the rate of tire depreciation savings at year y after rehabilitation

(8) Estimate repair and maintenance cost rate at pre-rehabilitation stage

(9) Estimate repair and maintenance cost rate at year y after rehabilitation

(10) Calculate the rate of repair and maintenance savings at year y after rehabilitation

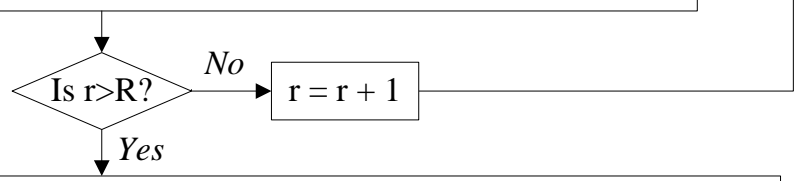

(11) Calculate total fuel consumption cost savings for entire network at year y after rehabilitation

(12) Calculate total tire depreciation cost savings for entire network at year y after rehabilitation

(13) Calculate total repair and maintenance cost savings for entire network at year y after rehabilitation

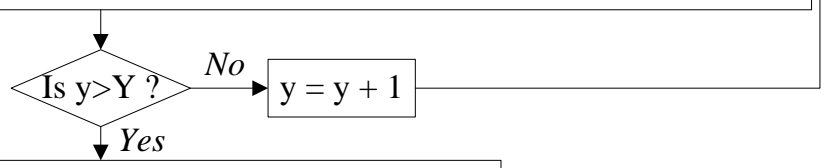

(14) Calculate total public benefits for the entire network

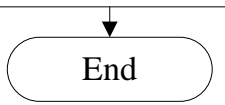

Figure 4-9 Public benefits calculation for transportation network implementation 
(9) Estimate the rate of repair and maintenance costs at the post-rehabilitation stage. In this step, the rate is estimated at any year over the lifespan period by using the associated equations mentioned in Table 3-3.

(10) Calculate the rate of repair and maintenance cost savings at year (y) over the lifecycle span. The results from the two previous steps are entered in Equation 4-13 to determine the cost savings rate. The calculation is repeated from steps 8 to 10 for each road section and each year of rehabilitation.

$$
\Delta \mathrm{MR}_{\mathrm{r}}^{\mathrm{y}}=\mathrm{MRI}_{\mathrm{r}}^{\mathrm{y}}-\mathrm{MRN}_{\mathrm{r}}^{\mathrm{y}}
$$

Where, $\Delta \mathrm{MR}^{\mathrm{y}} \mathrm{r}=$ rate of repair and maintenance savings of road section $(\mathrm{r})$ at year $(\mathrm{y})$ after rehabilitation; $\mathrm{MRI}_{\mathrm{r}}^{\mathrm{y}}=$ repair and maintenance rate of road section (r) at pre-rehabilitation conditions; and $\mathrm{MRN}_{\mathrm{r}}^{\mathrm{y}}=$ repair and maintenance rate of road section (r) after year (y) of rehabilitation.

(11) Calculate total fuel consumption cost savings for the entire network at year y after rehabilitation, by using Equation 4-14. The calculation is repeated over a predetermined lifecycle span $(\mathrm{y}=1$ to $\mathrm{Y})$. The total savings depend on number of traffic volume, length of the road section, and the rate of fuel consumption cost savings obtained from step 4.

$$
\mathrm{FS}^{\mathrm{y}}=\sum_{\mathrm{r}=1}^{\mathrm{R}} \mathrm{V}_{\mathrm{r}} * \mathrm{~L}_{\mathrm{r}} * \Delta \mathrm{FR}_{\mathrm{r}}^{\mathrm{y}}
$$

Where, $\mathrm{FS}^{\mathrm{y}}=$ total fuel consumption cost savings at year $\mathrm{y}$ after rehabilitation; $\mathrm{R}=$ number of road sections in the transportation network; $\mathrm{Y}=$ number of years to new rehabilitation effort; $\mathrm{V}_{\mathrm{r}}=$ traffic volume on road section $(\mathrm{r})$; $\mathrm{L}_{\mathrm{r}}=$ length of road section (r); and $\Delta \mathrm{FR}_{\mathrm{r}}^{\mathrm{y}}=$ rate of fuel consumption savings of road section (r) at year (y) after rehabilitation. 
(12) Calculate total tire depreciation cost savings for the entire network at year y after rehabilitation. Equation 4-15 can be used for estimating tire depreciation cost savings throughout the network. The calculation is repeated over a predetermined lifecycle span (y $=1$ to $\mathrm{Y}$ ). The total savings depend on number of traffic volume, length of the road section, and the rate of tire depreciation cost savings obtained from step 4.

$$
\mathrm{DS}^{\mathrm{y}}=\sum_{\mathrm{r}=1}^{\mathrm{R}} \mathrm{V}_{\mathrm{r}} * \mathrm{~L}_{\mathrm{r}} * \Delta \mathrm{DR}_{\mathrm{r}}^{\mathrm{y}}
$$

Where, $\mathrm{DS}^{\mathrm{y}}=$ total tire depreciation cost savings at year y after rehabilitation; and $\Delta \mathrm{DR}^{\mathrm{y}}{ }_{\mathrm{r}}$ $=$ rate of tire depreciation savings of road section $(\mathrm{r})$ at year $(\mathrm{y})$ after rehabilitation.

(13) Calculate total repair and maintenance cost savings for the entire transportation network at year y after rehabilitation, by using Equation 4-16. Similar to tire depreciation, the total savings of repair and maintenance costs are calculated based on traffic volume, length of the road project, and the rate of cost savings. Also, the calculation is repeated over the year of lifecycle.

$$
\mathrm{MS}^{\mathrm{y}}=\sum_{\mathrm{r}=1}^{\mathrm{R}} \mathrm{V}_{\mathrm{r}} * \mathrm{~L}_{\mathrm{r}} * \Delta \mathrm{MR}_{\mathrm{r}}^{\mathrm{y}}
$$

Where, $\mathrm{MS}^{\mathrm{y}}=$ total repair and maintenance cost savings at year y after rehabilitation; and $\Delta \mathrm{MR}^{\mathrm{y}} \mathrm{r}=$ rate of repair and maintenance cost savings of road section (r) at year (y) after rehabilitation (from step 10).

(14) Calculate total public benefits for the entire network by adding together the savings components estimated in steps 10-12. The summation is performed at each year after rehabilitation along the lifecycle span, and then the concept of net present worth is applied to find the total expected public benefits, as shown in Equation 4-17. 


$$
\mathrm{TPB}=\sum_{\mathrm{y}=1}^{\mathrm{Y}} \mathrm{FS}^{\mathrm{y}}(\mathrm{P} / \mathrm{F}, \mathrm{ir}, \mathrm{y})+\sum_{\mathrm{y}=1}^{\mathrm{Y}} \mathrm{DS}^{\mathrm{y}}(\mathrm{P} / \mathrm{F}, \mathrm{ir}, \mathrm{y})+\sum_{\mathrm{y}=1}^{\mathrm{Y}} \mathrm{MS}^{\mathrm{y}}(\mathrm{P} / \mathrm{F}, \mathrm{ir}, \mathrm{y}) \quad(4-17)
$$

Where, TPB = total expected public benefits; and ir = discount rate for the public benefit calculation (\%).

\subsection{Model Evaluation}

Two case studies are adopted in this section to evaluate the model performance and capabilities in evaluating the impact of rehabilitation on transportation networks. First example attempts to analyze and evaluate the total impacts of rehabilitation need in improving the conditions of transportation networks. Second example seeks to evaluate the impact of decision making related to treatment selection, implementation timing, and the length of analysis period on transportation networks.

4.8.1 Example 1: Evaluating the impact of rehabilitation need on transportation networks

In this section, the real transportation network data of the Florida's district 4 is analyzed in order to demonstrate the use and capabilities of the purposed models in evaluating the impacts of rehabilitation programs on transportation networks. The application example is designed with a hypothetical rehabilitation program applied to the Florida's state highway network in the area of district 4, which covers Broward, Palm Beach, Martin, St. Lucies, and Indian River County. Figure 4-10 presents the study area and transportation network in Florida's district 4 analyzed in this paper. There are about 144 road segments in the network under the state highway system with approximately 2,092 distance kilometers and 62 million vehicle-kilometers per day. 


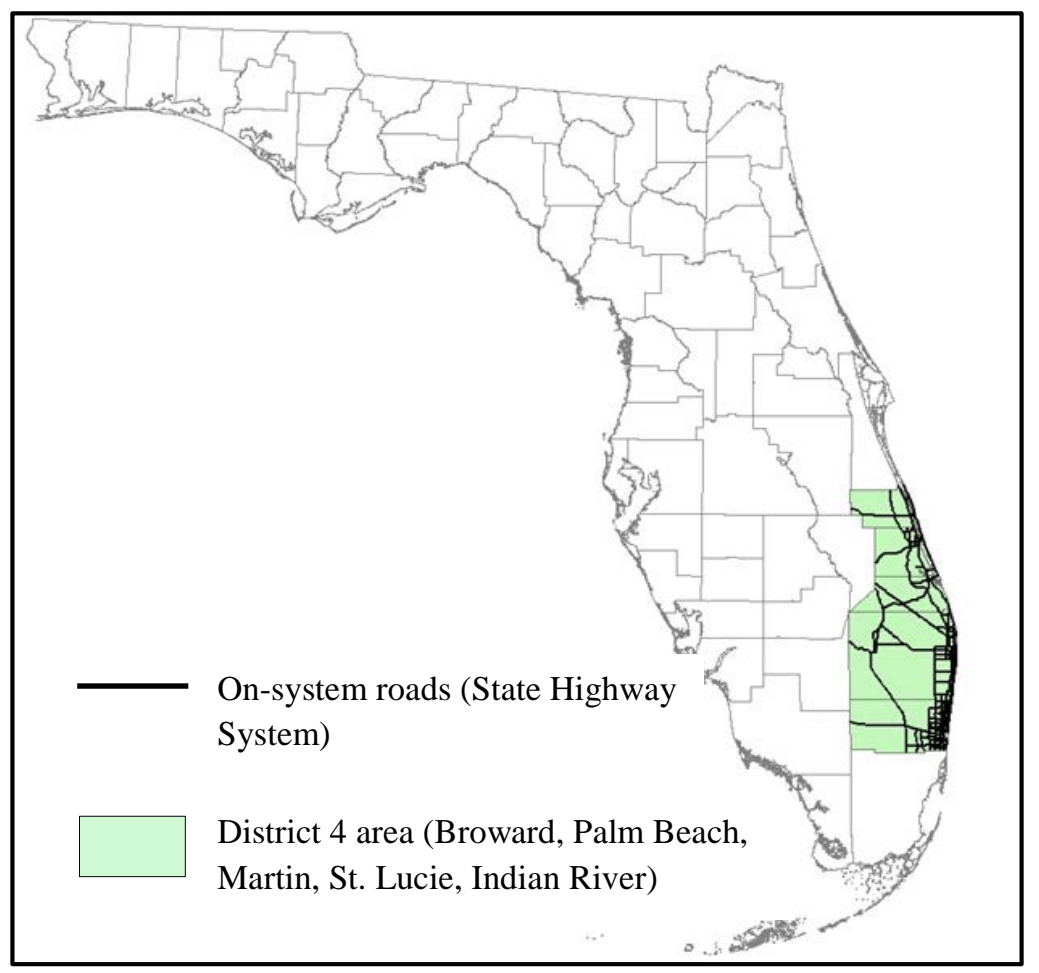

Figure 4-10 Florida district 4 state highway network

The rehabilitation program is assumed to repair and upgrade all roadways in the transportation network that are suffering from poor pavement conditions. All pavements with $I R I \geq 4 \mathrm{~m} / \mathrm{km}$ are selected to improve the pavement performance at many locations throughout the network. Therefore, 40 road segments are considered for rehabilitation in this section. All roadways in the network are assumed to be deteriorating over the 5-year program. The pavement conditions are taken into account the deterioration rate over time by adopting the equation from Paterson and Attoh-Okine (1992). To implement the example, the work zone speed limit during construction operations is assumed with 25\% reduction from the regular speed limit. Traffic volume is assumed to have $5 \%$ of light-duty and heavy-duty trucks with no change in overall traffic volume during construction. For simplicity, the construction duration is modified as 8 months based on the average 
construction duration mentioned in OECD (2005) with the impact of construction on all roadways throughout the network. The unit cost of travel delay is adopted from Copeland (1998) with an adjustment of the consumer price index from U.S. Bureau of Labor Statistics (Statistics 2014). The adjusted costs of travel delay are \$22.25 and \$40.64 per vehicle-hours for passenger cars and trucks, respectively. The total public benefits are calculated based on a 5\% discount rate net present value.

In this study, the impacts of rehabilitation programs are evaluated in terms of three main components - network energy consumption, public cost, and public benefits - by adopting the purposed models. The estimated total energy consumption of the example network is 14 trillion liters over the 5-year calculation period, which includes 1.44 billion liters during the construction and almost 14 trillion liters during the regular operation of the transportation network. This proportion of energy consumption between the construction and operation phase reveals a little amount of fuel consumed during construction comparing to the road usage phase. The expected pubic cost is estimated as 1.62 billion dollars resulting from the speed reduction of vehicles traveling through the work zone. Additionally, the public benefits are evaluated to be 284 million dollars over the 5-year program period. However, the total public benefits are expected to increase for a longer lifespan period.

\subsubsection{Example 2: Evaluating the impact of decision making in rehabilitation efforts}

In this section, the developed model is applied to part of the real transportation network covering the area of District 2, Florida, in order to demonstrate the model use and its capabilities in evaluating the impact of highway rehabilitation efforts on energy consumption of damaged transportation networks. The example consists of 27 road 
sections that are experiencing poor or worsening conditions of surface pavement deterioration. These road sections are assumed to be in need of rehabilitation treatments throughout many locations in the network over an analysis period. To this end, decision makers and planners are required to evaluate the impact on total network energy consumption as a result of selecting specific rehabilitation treatments. Table 4-7 summarizes the data of these 27 candidate road sections, which include the length of section, average daily traffic volume (AADT), average truck traffic volume (AATA), pavement conditions (in terms of IRI), traveling speed, and number of lanes on a road section. Figure 14-11 illustrates the transportation network in this example covering all rehabilitation-needed road sections. Please note that all road sections here are contained in the national highway systems.

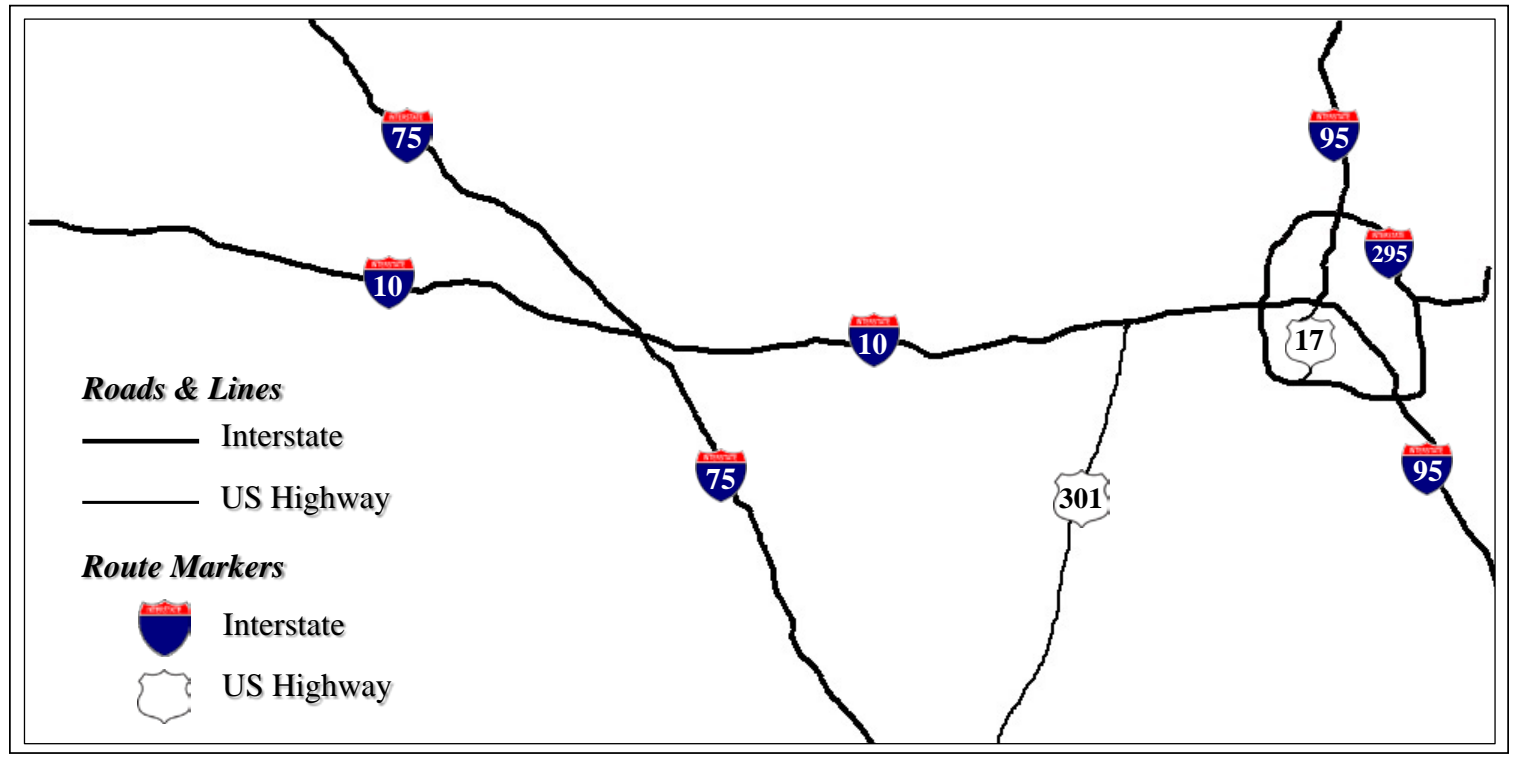

Figure 4-11 Case study of the damaged transportation network 
Table 4-7 Candidate Rehabilitation Projects

\begin{tabular}{ccccccc}
\hline $\begin{array}{c}\text { Road } \\
\text { section }\end{array}$ & $\begin{array}{c}\text { Length } \\
(\mathrm{km})\end{array}$ & $\begin{array}{c}\text { AADT } \\
\text { (veh/day) }\end{array}$ & $\begin{array}{c}\text { AATA } \\
\text { (veh/day) }\end{array}$ & $\begin{array}{c}\text { IRI } \\
(\mathrm{m} / \mathrm{km})\end{array}$ & $\begin{array}{c}\text { Traveling } \\
\text { speed }(\mathrm{km} / \mathrm{h})\end{array}$ & $\begin{array}{c}\text { Number } \\
\text { of lanes }\end{array}$ \\
\hline 1 & 3.4 & 2,300 & 115 & 3.5 & 72.4 & 2 \\
2 & 55.9 & 73,000 & 5,329 & 3.5 & 104.6 & 3 \\
3 & 56.6 & 56,000 & 10,472 & 4.5 & 112.7 & 3 \\
4 & 5.9 & 33,500 & 7,906 & 4 & 112.7 & 3 \\
5 & 53.1 & 21,500 & 6,300 & 4 & 112.7 & 2 \\
6 & 49.0 & 33,500 & 7,906 & 4 & 112.7 & 3 \\
7 & 7.9 & 15,900 & 3,800 & 3.5 & 72.4 & 2 \\
8 & 41.1 & 26,346 & 5,743 & 4 & 112.7 & 2 \\
9 & 33.3 & 20,540 & 4,991 & 4 & 112.7 & 2 \\
10 & 46.3 & 34,000 & 8,568 & 3.5 & 112.7 & 3 \\
11 & 40.9 & 20,000 & 6,780 & 3.5 & 112.7 & 2 \\
12 & 1.9 & 11,400 & 1,756 & 3.5 & 56.3 & 2 \\
13 & 3.7 & 48,000 & 912 & 3.5 & 88.5 & 2 \\
14 & 10.3 & 31,000 & 651 & 4.5 & 48.3 & 2 \\
15 & 16.9 & 60,000 & 10,260 & 4 & 112.7 & 3 \\
16 & 2.8 & 11,000 & 924 & 4 & 56.3 & 2 \\
17 & 17.0 & 79,500 & 6,281 & 3.5 & 88.5 & 4 \\
18 & 5.6 & 27,000 & 567 & 4 & 56.3 & 3 \\
19 & 41.0 & 58,500 & 8,015 & 4 & 104.6 & 2 \\
20 & 27.1 & 116,500 & 8,505 & 4 & 104.6 & 3 \\
21 & 11.8 & 14,000 & 3,948 & 4 & 96.6 & 2 \\
22 & 0.9 & 43,000 & 5,891 & 4 & 104.6 & 3 \\
23 & 1.1 & 20,000 & 6,780 & 3.5 & 112.7 & 2 \\
24 & 19.7 & 60,000 & 10,260 & 4.5 & 112.7 & 3 \\
25 & 56.1 & 81,000 & 9,801 & 3.5 & 112.7 & 3 \\
26 & 34.5 & 107,000 & 5,243 & 3 & 72.4 & 3 \\
27 & 8.9 & 16,300 & 4,597 & 3.5 & 104.6 & 2 \\
\hline & & & & & &
\end{tabular}

The construction cost of each project is estimated by adopting the average cost per lane- mile from Irfan (2010). Similarly, the historical data is applied to estimate the construction durations for thin overlay (from OECD 2005), and for rehabilitation (from Caltrans 2015). The durations for thick overlay and reconstruction activity are estimated by using interpolate and extrapolate operation, respectively, based on the construction 
durations of the two treatments previously mentioned. In this section, the model was not only evaluated its capabilities, but also used to analyze the impact of rehabilitation efforts based on the following questions: (1) What is the impact of the rehabilitation treatment selection on network energy consumption and pavement conditions? (2) How does the timing of pavement implementation affect the total energy consumption and pavement conditions of transportation networks? (3) How does the length of analysis period affect the trend of energy consumption and pavement conditions from different rehabilitation treatment strategies? The following subsections provide the analyses and answers to address these three questions.

\subsubsection{Impact of Rehabilitation Treatment Selection}

This subsection presents the impact of the rehabilitation treatment strategy on energy consumption and pavement conditions of transportation networks. Two types of rehabilitation strategies are assumed for the comparison: (1) the low-cost rehabilitation treatment, and (2) the high-cost rehabilitation treatment. In this study, the types of treatment will be assigned based on the current pavement conditions (IRI). For instance, the HMA overlay structural and reconstruction will be designated for a pavement with the IRI greater than $4 \mathrm{~m} / \mathrm{km}$. Since the HMA overlay has a lower unit cost of rehabilitation than reconstruction activity, the HMA overlay is considered as the low-cost treatment strategy while the high-cost treatment for the reconstruction. This consideration was similarly applied for all road pavements to identify the types of rehabilitation strategies.

The analysis was performed over a 20-year planning period, which is composed of four 5-year highway rehabilitation programs. In this subsection, the treatment is assumed 
to be implemented on each road pavement at the first year of each rehabilitation program. The influence from the year of implementation is neglected in this part of analysis. The result shows that a selection of rehabilitation treatment has a significant impact on overall pavement conditions (in terms of average IRI) and therefore network energy consumption.

In this application example, it shows that pavement conditions have a very high correlation with energy consumption. As illustrated in Figure 4-12, worsening pavement conditions lead to an increase in energy consumption of pavement. This means the level of severity on environmental impact can be larger if an improvement of pavement conditions is insufficient. In addition, the numerical analysis was simply employed to investigate a correlation of the factors in this analysis section. The result presents the non-linear pattern of the relationship between IRI and energy consumption, which identifies that a decrease of $1-\mathrm{m} / \mathrm{km}$ IRI on different roads or transportation networks is not necessary to always provide the same magnitude of the reduction in energy consumption.

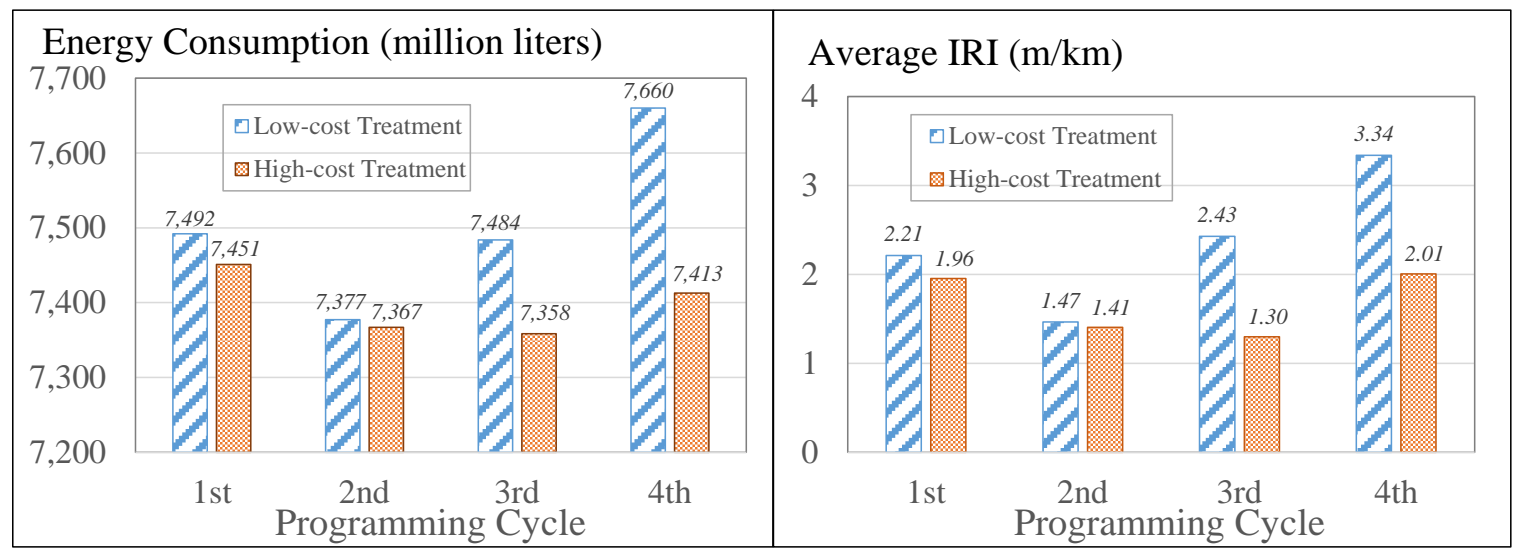

(a)

(b)

Figure 4-12 Impact of rehabilitation treatment selection on (a) network energy consumption; and (b) overall network pavement conditions 
Further analysis was performed to consider the effect of different rehabilitation strategies as a result of variations in rehabilitation budget allocated to transportation networks (see Figure 4-13). As the cost has an inverse relationship to pavement performance (Fwa and Sinha 1991), a pavement typically requires good construction quality and techniques, which need a high level of funding, to have better pavement conditions (Wang et al. 2003). As a result, the different levels of rehabilitation budget can lead to the differences in future pavement performance (Liu and Wang 1996) and therefore total energy consumption. However, the effect of budget allocation tends to be larger for the earlier-implemented programs in this study, with respect to initial pavement conditions at the time when pavement is rehabilitated. For example, high budgets are needed for repairing the transportation network during the first rehabilitation program due to poor or very poor conditions of surface pavements. The second program then tends to require much less funds since pavement conditions are dramatically improved from the first-cycle implementation. The conditions of the pavements still continue improving through the third program as a result of the ongoing pavement improvement from the previous programs. The pavement treated with the low-cost strategy tends to have a lower level of performance than the high-cost scenario. After the first-cycle implementation, the variations of overall pavement conditions and network energy consumption between the two scenarios will expand as the impact of treatment is accumulated through the analysis planning period. 


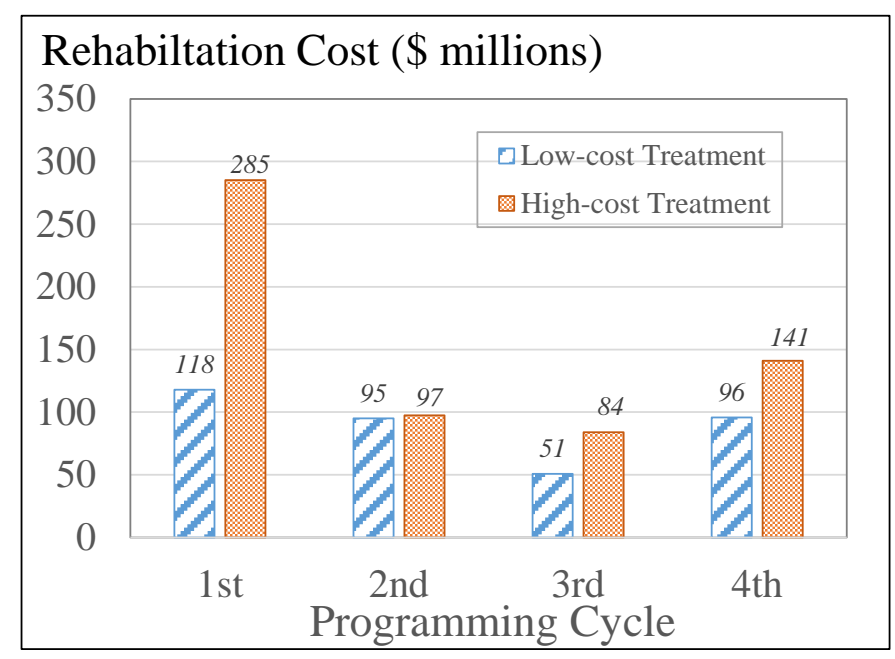

Figure 4-13 Total rehabilitation budget needed for each scenario

\subsubsection{Impact of Pavement Implementation Timing}

In this subsection, the analysis is executed to investigate whether the timing of pavement implementation along an analysis period has an impact on energy consumption and overall pavement conditions of transportation networks. The consideration takes place each year of a 5-year programming timespan. The result tends to show the significance of the implementation timing with a lower network energy consumption and better overall pavement conditions in an early-implemented rehabilitation program. As shown in Figure 4-14, the transportation network is likely to have less overall IRI when all candidate pavements are applied at the beginning of the rehabilitation program. The overall network pavement conditions will worsen if the rehabilitation effort is postponed. Considering the project level, the road section with an early implementation tends to recover its conditions better and sooner than the late-implemented pavement. The similar effect also enlarges the total energy consumed throughout the transportation network. In addition, a rehabilitation treatment applied at any year of the program has a significant impact on a selection of the 
next-program treatment. For instance, the initial conditions of the pavements in this example mostly range from the poor to very poor levels. Based on its current conditions, the pavement will be treated from the list of candidate treatment options. The overall network performance considerably improves after the first-year implementation. However, the pavement typically continues deteriorating as a result of several factors, such as traffic and weather conditions. The pavement therefore shows a worse condition when the rehabilitation is delayed. For the second-year implementation in this example, the reconstruction is mostly selected for the high-cost treatment strategy, while a lowerefficient method (e.g. thick HMA overlay) is selected in the low-cost strategy. Since the reconstruction has a very high construction cost, it shows a very wide range of cost difference between two treatment scenarios with a few variation in the IRI values and energy consumption. For a later implementation, an improvement in pavement conditions and a reduction in energy consumption does not significantly differ among the scenarios. This is because the treatment alternative applied on a pavement is mostly similar in both scenarios. This similarity is more coincident as the timing of implementation increases. It is noteworthy that only reconstruction is applied after the second year of the rehabilitation program for the high-cost treatment scenario since the conditions of the pavements fall beyond an acceptable performance threshold and the reconstruction is the only effective option for recovering pavement conditions. 


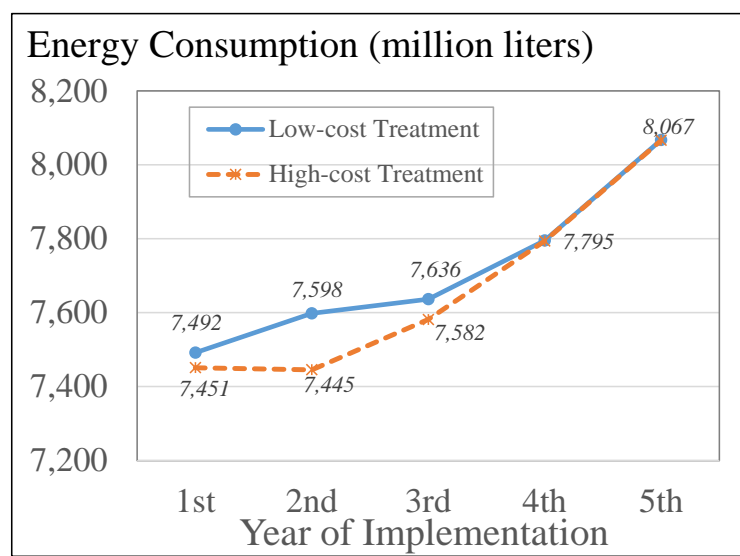

(a)

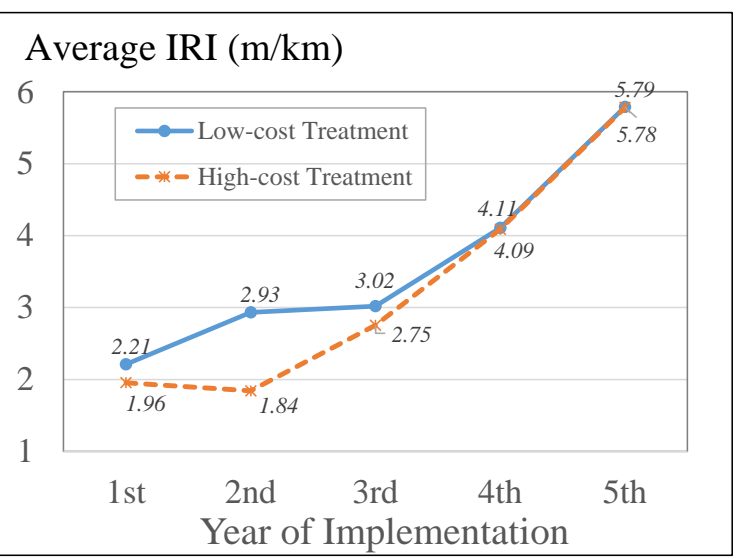

(b)

Figure 4-14 Impact of rehabilitation treatment timing on

(a) network energy consumption; and (b) overall network pavement conditions

As aforementioned, with the effects of rehabilitation treatment alternatives and the timing of implementation, network energy consumption will be varied if the rehabilitation treatments are differently applied over the pavements. Figure 4-15 represents an estimated saving in total energy consumption per one dollar of rehabilitation cost spent over the transportation network. The savings in energy consumption are largest when all pavements are implemented during the first year of the rehabilitation program. The magnitude of the savings decreases if the rehabilitation is postponed to the following years of the program. However, the ratios of the savings are not significantly different due to the similarity in a selection of rehabilitation treatment alternatives between two scenarios after the secondyear implementation. 


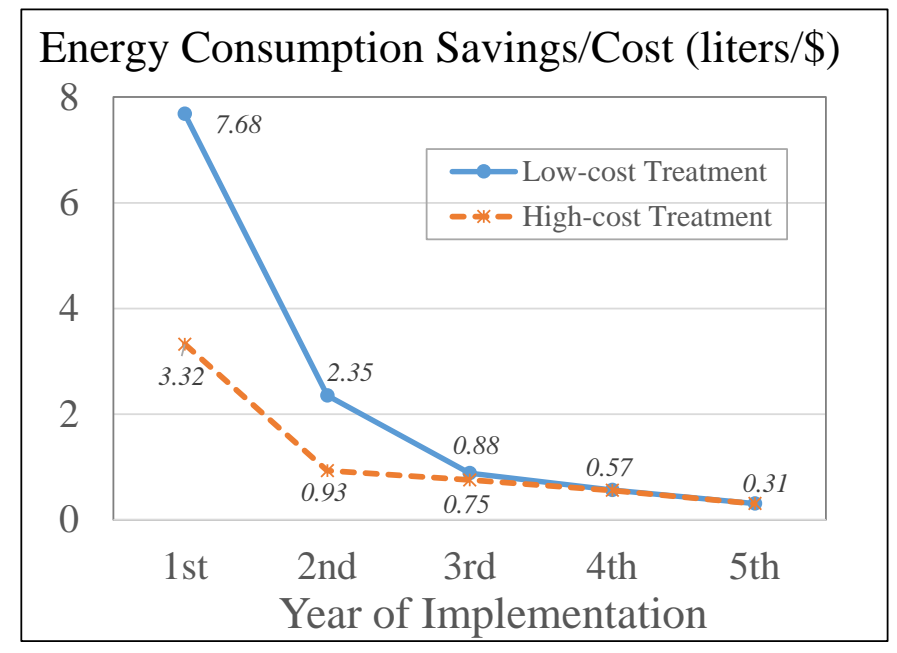

Figure 4-15 Effect of rehabilitation cost on energy consumption at any year of implementation

\subsubsection{Impact of Analysis Period}

This subsection investigates if the length of an analysis period will change the selection of rehabilitation treatment strategy. The analysis is assumed to be calculated at a 5-year, 10-year, 15-year and 20-year timespan to observe the impact on decisions of state highway agencies in selecting and implementing different rehabilitation treatments on damaged transportation networks. Within the analysis period, a selection on rehabilitation treatments will be performed at the beginning of every 5 years. Figure 4-16 illustrates total network energy consumption when the analysis timespans are considered for 5 years, 10 years, 15 years and 20 years. This subsection does not aim to numerically compare energy consumption and overall pavement performance, but only analyze whether the length of an analysis period will make changes on the rehabilitation decision making. The finding demonstrates that the length of the analysis period does not affect the decisions of decision makers when considering network energy consumption (see Figure 4-16). The bar chart shows a higher energy consumption if the low-cost treatment strategy is adopted for 
rehabilitation regardless of the length of the analysis. The difference in energy consumption between the two treatment scenarios is very small. The magnitude of this difference somehow expands with an increase in the length of the analysis period, due to an accumulated impact from the previous program implementation. For the overall pavement conditions, the bar chart from Figure 4-12(b) can present the impact with a higher average IRI from implementing the low-cost treatment scenario on the transportation network. It should be noted that an order of programming cycle shown in Figure 4-12(b) is related to the length of analysis period. For instance, the $1^{\text {st }}$ program cycle can be comparable to a 5year length of the planning period, the $2^{\text {nd }}$ program for a 10 -year length, and so on. In addition, all IRI values shown in Figure 4-12(b) are overall pavement conditions averaged from the multiplication of the anticipated IRI and the length of all candidate road sections by the total length of the transportation network.

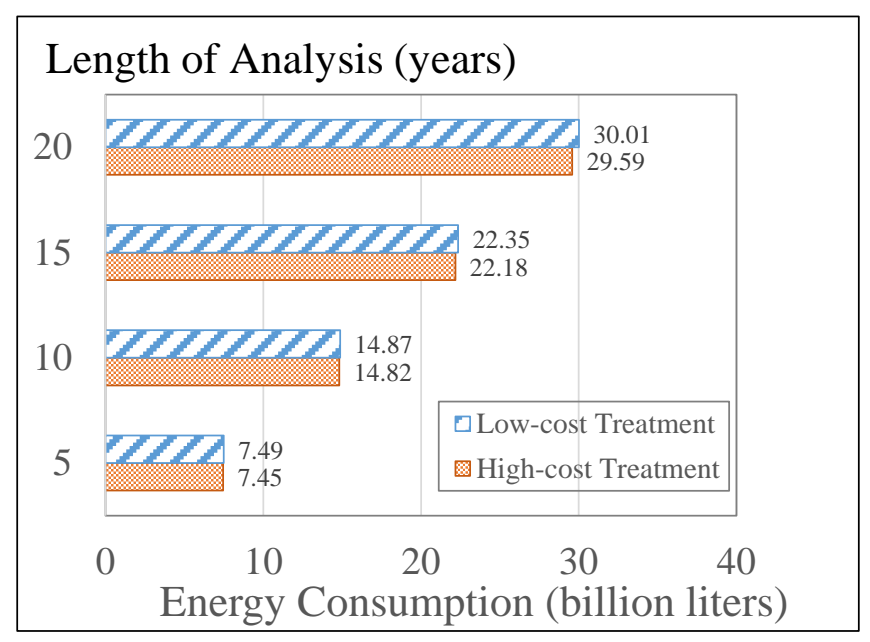

Figure 4-16 Impact of length of analysis period on network energy consumption 


\subsection{Chapter Summary}

A novel model is developed in this chapter to support an evaluation of economic and environmental impacts on transportation networks as a result of highway rehabilitation decision making. The developed model is composed of five main modules with the capabilities of: (1) identifying candidate rehabilitation treatment alternatives to a deteriorating pavement; (2) evaluating and forecasting the impact of rehabilitation treatments on pavement performance; (3) estimating total energy consumption throughout the entire network as a result of highway rehabilitation decisions; (4) estimating the public costs as a result of the vehicle travel-delay from the speed reduction during the construction operations, and (5) evaluating the impact of rehabilitation decision making on public benefits as the expected savings in road user costs after rehabilitation. The model's performance and capabilities are proved with an analysis of an application example based on the real transportation network. The finding in this chapter can be used to improve and support the decision making process in highway rehabilitation in order to serve the economic and environmental platforms. The developed model will be expanded in the next chapter with an integration of an optimization technique in order to generate an effective and environmental-support rehabilitation program(s) that can enhance an achievement of sustainability goal in deteriorating transportation networks. 


\section{CHAPTER 5}

\section{OPTIMIZING HIGHWAY REHABILTATION PROJECTS}

\subsection{Introduction}

The main objective of this chapter is to present the development of an environmentally-friendly decision-support model for planning highway rehabilitation programs. Three main questions are identified to achieve this research objective: (1) What are the main decision variables that should be modeled and optimized to minimize $\mathrm{CO}_{2}$ emissions and maximize net public benefits? (2) How can the impacts of the decision variables and constraints on the optimization objectives be modeled and analyzed? (3) Which optimization technique is best suited to search for and identify optimal highway rehabilitation program(s) to this study? This chapter is written in such a way that answer aforementioned research objectives. The sections will describe the concept of the developed multi-objective optimization model, decision variables, planning objectives, and optimization constraints. The last section will also demonstrate the performance and capabilities of the developed model in planning rehabilitation efforts.

\subsection{Multi-Objective Optimization Model}

In order to address the research questions, this chapter presents the development and application of a new optimization model, which is capable of: (1) identifying candidate rehabilitation treatment alternatives to deteriorating pavements; (2) evaluating the impact of rehabilitation efforts on pavement performance; (3) estimating total $\mathrm{CO}_{2}$ emissions as a result of highway rehabilitation decisions in transportation networks; (4) evaluating the cost of travel delay due to construction operations; (5) quantifying the expected savings in 
road user costs resulting from rehabilitation decisions; and (6) optimizing rehabilitation efforts to search for and identify highway rehabilitation program(s) that simultaneously minimize $\mathrm{CO}_{2}$ emissions and maximize net pubic benefits under the limited funding. Figure 5-1 demonstrates the concept of highway rehabilitation programming and the optimization model proposed in this paper.

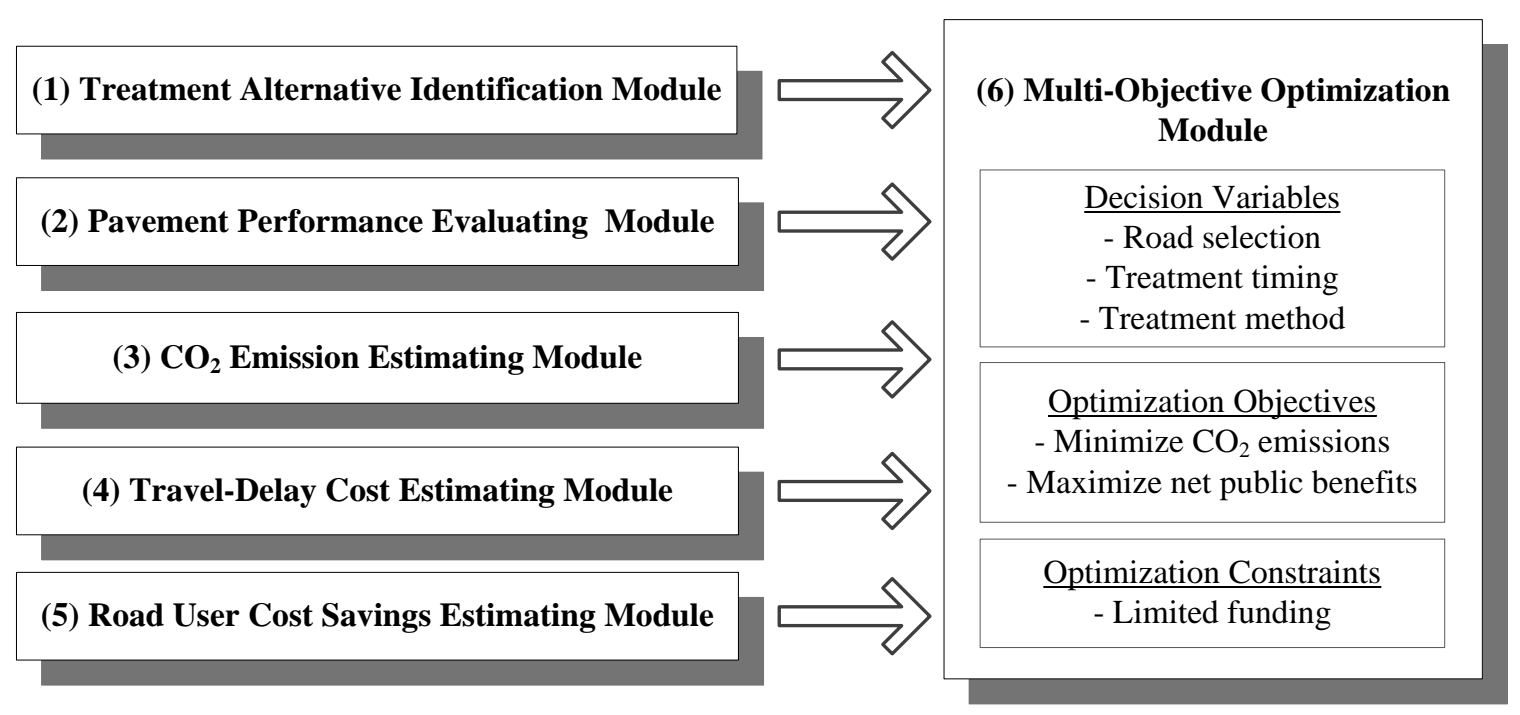

Figure 5-1 Highway rehabilitation programming and optimization model

There is typically a wide range of alternatives in decision making for highway construction programs. Each of these alternatives has a different outcome on the environmental impact and public benefits. It is therefore important for planners and decision makers to be able to search for and identify the construction program(s) that can minimize the environmental impact and maximize public benefits, simultaneously. This is a multi-criteria and constrained optimization problem that should be modeled. The decision variables and planning objectives of this optimization problem should be modeled in an effective and efficient manner. In addition, the optimization objectives are nonlinear and 
non-continuous, which require the selection of a suitable multi-objective optimization technique capable of handling such problems.

This model presents the optimization of highway rehabilitation efforts for deteriorating transportation networks in order to satisfy two main planning objectives: (1) minimizing $\mathrm{CO}_{2}$ emissions on transportation networks; and (2) maximizing net public benefits. Accordingly, the model is designed to consider three main decision variables: (1) road selection (selecting which road sections to undergo rehabilitation); (2) treatment timing (determining the timing that the pavement will be treated); and (3) treatment method (identifying the treatment method will be applied on each aging pavement). These three decision variables are included in the optimization operation to identify the optimal highway rehabilitation program(s) that is capable of minimizing $\mathrm{CO}_{2}$ emissions while maximizing net public benefits subject to budget constraints.

\subsubsection{Optimization Operations}

Figure 5-2 represents the operational scheme of the multi-objective optimization model developed in this paper. A set of population solutions is randomly generated. Each solution represents the combination of decision variables. This combination contains the different chromosomes that each will represent as the random number of the decision variable corresponding to each rehabilitation pavement section. For instance, the analysis requires a total of 30 chromosomes if ten competing rehabilitation pavement sections are considered under a 5-year programming horizon. Figure 5-3 illustrates the string of these 30 chromosomes that combine all decision variables of all competing road sections. These generated solutions are then used to verify with the identification of treatment alternatives. The pavement performance of each road section is measured and forecasted afterwards as 
a result of the randomly generated population. The solution's fitness is then evaluated by calculating $\mathrm{CO}_{2}$ emissions and net public benefits on the transportation network. Net public benefits are basically related to the estimations of travel-delay cost and road user cost savings, in which their details will be described in the optimization planning objective sections. The genetic algorithm is then conducted by the operators of sorting, selecting, crossover, and mutation to generate a new set of better population solutions for the next running operation. The procedure is repetitively performed for a predefined number of generations or until the error between two successive generations is smaller than a predefined tolerance. The optimal/near optimal solutions can be eventually obtained from the final set of population after the completion of genetic algorithm operations.
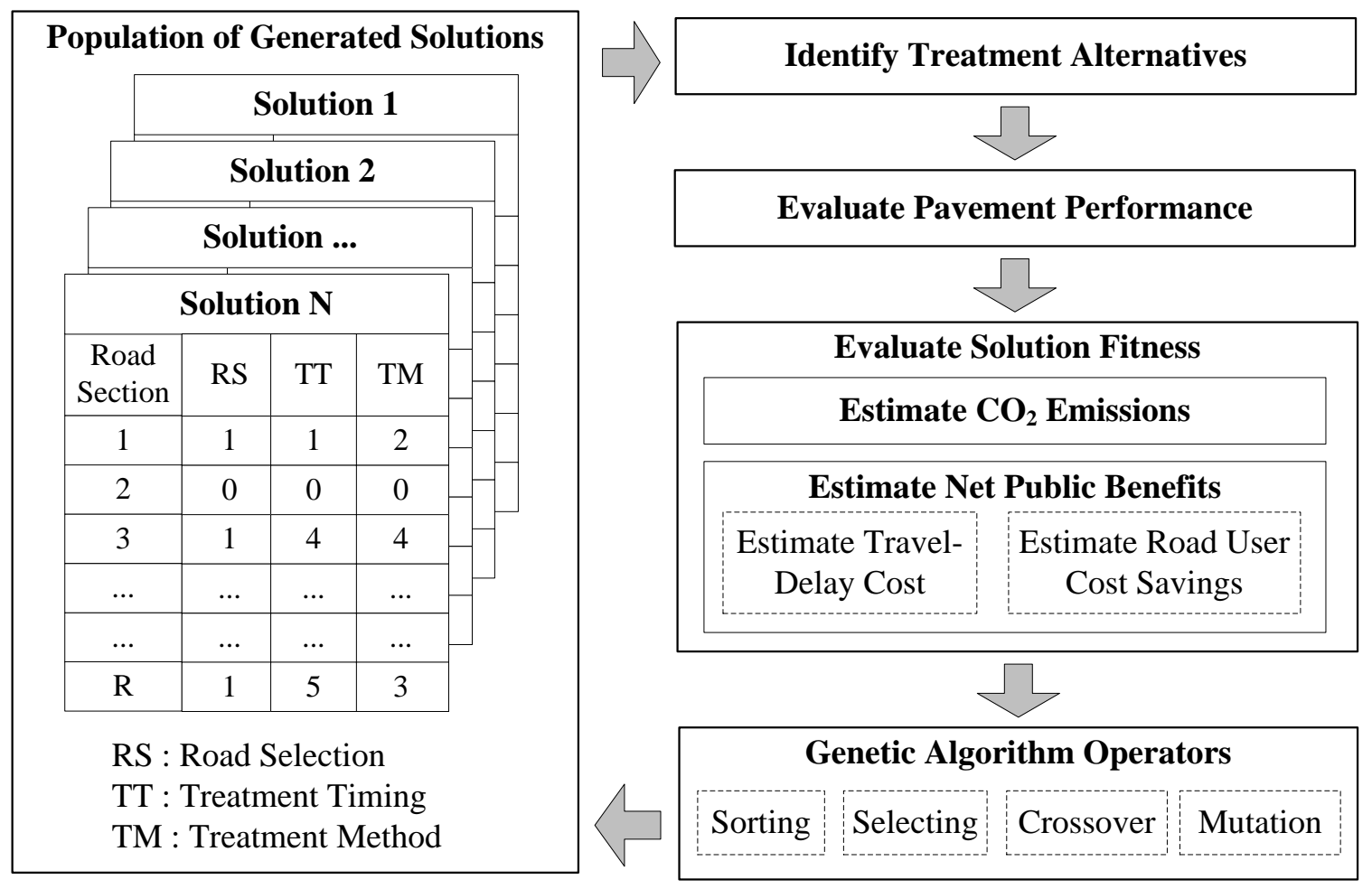

Figure 5-2 Multi-objective optimization model 


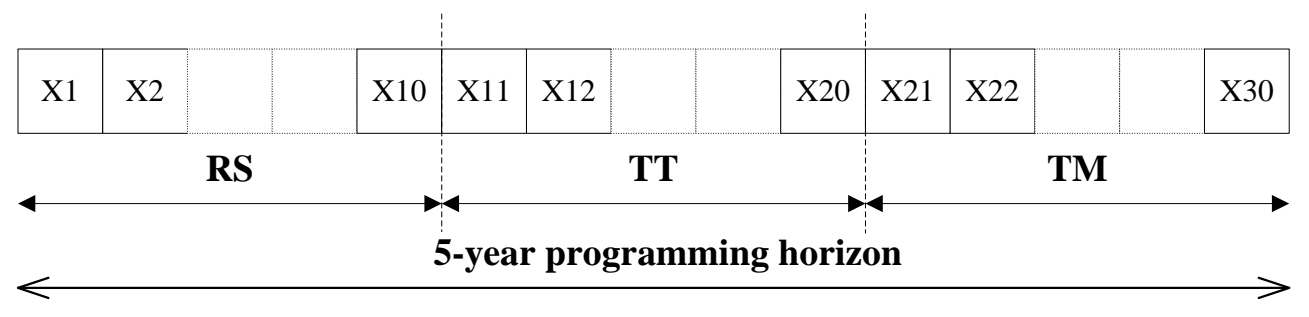

Figure 5-3 Population solution for 10 road sections under a 5-year programming horizon

\subsubsection{Optimization Search Engine}

The Non-dominated Sorting Genetic Algorithm II (NSGA-II) developed by Deb et al. (2002) is utilized to solve the optimization problem in this study. NSGA-II is the most superior evolutionary algorithm nowadays that is capable of searching for and identifying the optimal/near optimal solution (s), which satisfy the planning objectives and optimization constraints. To deal with the problem in this study, NSGA-II is the most suitable, with its capabilities in overcoming several challenges: (1) the multi-objective nature; (2) the nonlinear and non-continuous objective functions; and (3) the huge search space. First, this study deals with the multi-objective problem that is subjected to minimizing $\mathrm{CO}_{2}$ emissions and maximizing net public benefits simultaneously. However, the conventional optimization approach is struggling to handle the type of the multiobjective nature problem, as presented in this research. Second, the objective functions in evaluating the impact of highway rehabilitation decisions on $\mathrm{CO}_{2}$ emissions and net public benefits are discontinuous and nonlinear. This prevents the validity in using the traditional optimization method that is able to cope with the linear function. Third, the optimization problem in this study requires very enormous search space in generating the optimal/near optimal solution(s). For instance, in a multi-objective optimization problem with only ten 
candidate rehabilitation road sections, the search space will be as large as $(60)^{40}$, which includes more than $1.3 \times 10^{71}$ possible solutions in the analysis.

This study employs the recent constraint-implementation version of NSGA-II coded in Matlab, named NGSM version 1.4, developed by Song (2011). The code was incorporated with the newly written function statements that define all optimization objective functions and constraints specific to the problem in this study. The following sections will describe the decision variables, optimization constraints, and objective functions that are integrated in building the highway rehabilitation programming and optimization model.

\subsection{Decision Variables}

As mentioned before, three main decision variables (i.e. road selection, treatment timing, and treatment method) are optimized to search for optimal highway rehabilitation program(s) in this paper. The detailed description of each decision variable and its anticipated impact on transportation networks will be given as follows.

\subsubsection{Road Selection (RS)}

Decision makers are typically limited to select only a subset of the entire road sections due to the limitation in available funds and budget of transportation agencies. Since each road section has its own specific characteristics, selecting which road will be rehabilitated therefore has a very significant impact on a transportation network. For instance, selecting to repair a road with a high traffic volume can have a higher savings in the road user costs compared to a road with a lower traffic volume. On the other hand, a high-traffic road will cause a very significant level of energy consumption due to the 
operations of all vehicles traveling on the road, compared to a road with a lower usage level.

In this model, road selection is assigned as a binary variable that will be 1 for selected road sections and 0 for the road sections that were not selected. Figure 5-4 illustrates all chromosomes $\mathrm{X}$ of a generated solution for upgrading $\mathrm{R}$ road sections under a 20-year predefined analysis period with four 5-year highway rehabilitation programs, and the possible decision variables associated to each chromosome.

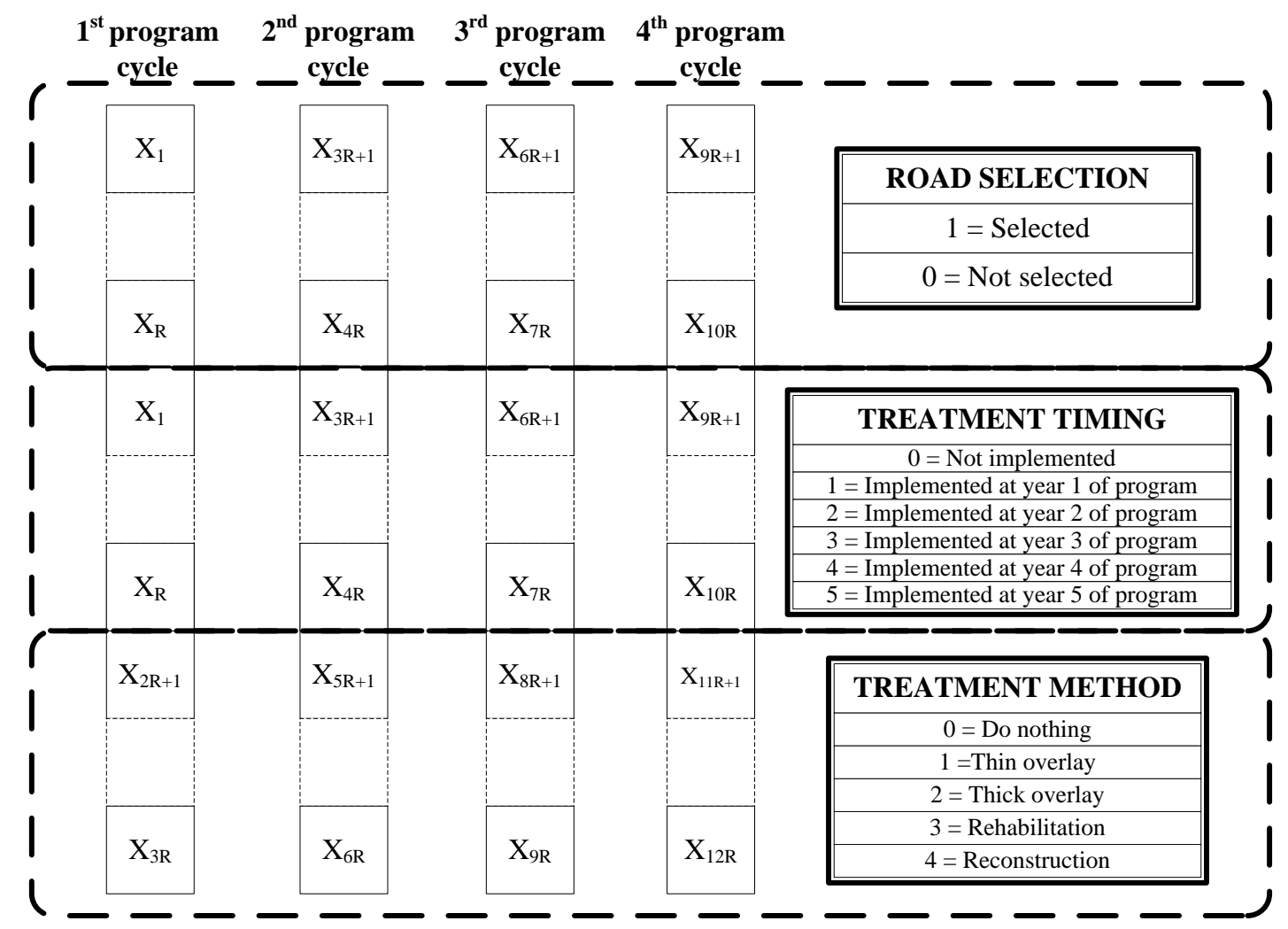

Figure 5-4 Population solution and decision variables under a 20-year horizon plan

\subsubsection{Treatment Timing (TT)}

The timing of when a rehabilitation treatment is applied to a road has a significant effect on pavement conditions. This means different timings of treatment application will 
also affect energy consumption, $\mathrm{CO}_{2}$ emissions and road user costs of transportation networks. With a delayed treatment application, pavement conditions of a road will get worse, resulting a high level of energy consumption and $\mathrm{CO}_{2}$ emissions, and road user costs. On the other hand, a scarcity of financial resources and an increase of traffic congestion may arise if many road sections are scheduled to receive treatment concurrently.

In this model, treatment timing represents a schedule of a road section to receive the pavement treatment. It is coded as an integer variable, ranging from 0 to 5 , which represents the year of the highway rehabilitation program at which the treatment is scheduled for application. For example, 0 represents no implementation for a road section, 1 represents a road section is scheduled to receive treatment at year 1 of the rehabilitation program, and so on. Figure 4 also represents how the treatment timing variable can be defined in a population solution.

\subsubsection{Treatment Method (TM)}

The selection of the pavement treatment methods has a direct and significant impact on the pavement performance. From the literature, the type of treatment applied on the pavement has different effect on performance jump and post-treatment performance (Irfan 2010; Irfan et al. 2008; Lamptey et al. 2008; Wang et al. 2003). In addition, current conditions of the pavement can challenge the selection of the candidate treatment methods. For instance, a road with relatively new conditions with very little damage requires no or only minor maintenance. However, reconstruction may be the only feasible option for improving the pavement conditions if the pavement is badly deteriorated with very significant damage (Carnahan et al. 1987). The past literature shows the correlation between highway treatment method and a level of energy consumed throughout a 
transportation network (Limsawasd et al. 2016; Zhang et al. 2012), and therefore $\mathrm{CO}_{2}$ emissions. The higher-cost and intensive treatment will lead to less energy consumption as a consequence of a better improvement in pavement conditions, and provide lower net public benefits as the balance between road user cost savings and rehabilitation cost (Limsawasd et al. 2016).

In this model, pavement treatment method is identified according to the selected rehabilitation treatment applied on the pavement. In this study, the variable is assigned as an integer number varying between 0 and 4, as shown in Figure 4. For example, 0 represents "Do nothing”, 1 represents “Thin overlay”, 2 represents “Thick overlay”, 3 represents “Rehabilitation”, and 4 represents “Reconstruction”. However, decision makers can redesign the options of this variable upon their preferences and current practices.

\subsection{Optimization Planning Objectives}

The model in this study is designed to optimize rehabilitation efforts to satisfy two planning objectives: (1) minimizing $\mathrm{CO}_{2}$ emissions; and (2) maximizing net public benefits of deteriorating transportation networks. To achieve these two main objectives, five modules are constructed to support an evaluation of the impact from highway rehabilitation decisions on $\mathrm{CO}_{2}$ emissions and public benefits in transportation networks. These modules include: (1) treatment alternative identification module; (2) pavement performance evaluating module; (3) $\mathrm{CO}_{2}$ emission estimating module; (4) travel-delay cost estimating module; and (5) road user cost savings estimating module. Figure 5-5 presents the calculation algorithm and how the modules are used and linked to establish the final outcomes. 
The steps of calculation are started with the treatment alternative identification module to identify candidate treatment options for deteriorating pavement under different conditions. After that, the calculation can be categorized into two main aspects - during construction operations and regular operation. Two main components are taken into consideration for evaluating the impact during the construction in this study. The first component is $\mathrm{CO}_{2}$ emissions as a result from a reduction in network energy consumption due to a reduced average speed of vehicle travelling through the construction work-zone. This component can be measured by using the $\mathrm{CO}_{2}$ emission estimating module. The second component is an increased travel cost as a result of an expected travel delay during the period of treatment application. This cost component can be estimated by using the travel-delay cost estimating module.

In addition, the impact of highway rehabilitation decisions during the regular operation phase is categorized into two main elements: (1) $\mathrm{CO}_{2}$ emissions generated from vehicles traveling on the improved pavement after applying rehabilitation treatment; and (2) an expected savings in road user costs resulting from better surface conditions of the treated pavement. This calculation will initiate the predicted pavement performance curve over time by using the pavement performance evaluating module. The pavement conditions in terms of the international roughness index (IRI) will be forecasted as a result of implementing the selecting pavement treatment. Accordingly, $\mathrm{CO}_{2}$ emissions and road user cost savings can be estimated by using the $\mathrm{CO}_{2}$ emission estimating module and road user cost savings estimating module, respectively. To this end, the net public benefits of highway rehabilitation programs can be calculated, by applying the concept of net present value, as the balance of cost of travel 


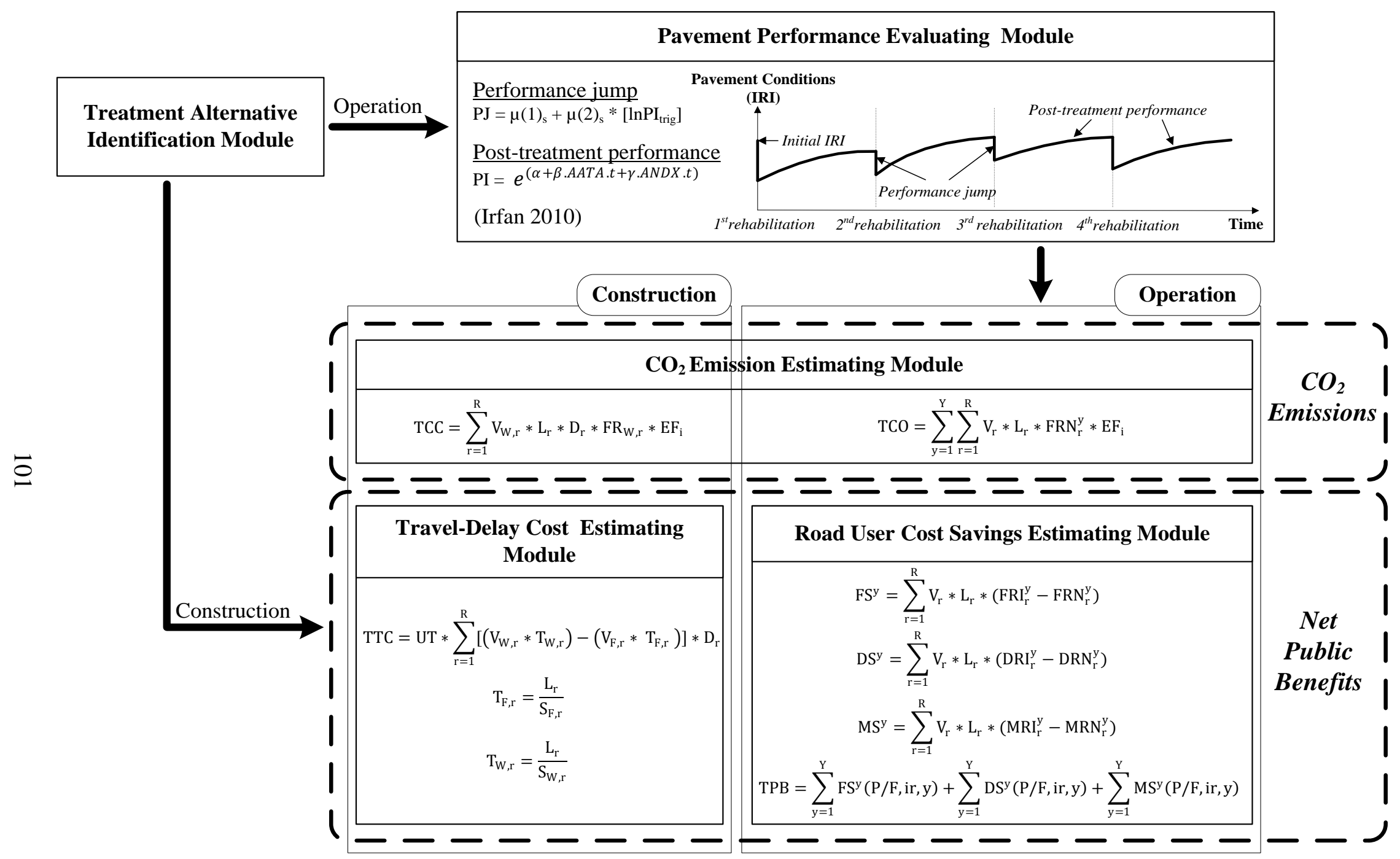

Figure 5-5 Calculation algorithm in evaluating the impact of highway decisions on optimization planning objectives 
delay, savings in road user costs, and cost of rehabilitation at a predefined discount rate. The details for all modules are described in the following subsections.

\subsubsection{Treatment Alternative Identification Module}

Decision makers are typically required to select the most effective treatment method and the optimal timing of treatment application. Generally, the selection of pavement treatment alternatives will depend on current surface conditions and expected improved conditions after treatment. This module is developed to support state departments of transportation in identifying candidate treatment options for surface pavement deteriorating under various damaged conditions. The past literature was investigated to create the rehabilitation treatment alternative identification scheme that is practicable and best-suited for the case study in this study. The developed scheme provides candidate treatment alternatives depending on the current level of damage and the targeted conditions of pavement after improvement, which are identified in terms of IRI in this study. The detail of how the rehabilitation treatment alternative identification scheme was developed can be found from chapter 4. However, it is noteworthy that this developed scheme was based on only some sources of existing studies and it can be redesigned afterwards as per the current practices of highway transportation agencies.

\subsubsection{Pavement Performance Evaluating Module}

As pavement condition is one of the significant factors that affects total energy consumption and public benefits of highway transportation networks, the forecast of future surface conditions and the prediction of the impact from the selected rehabilitation treatment is very critical. This module has an objective to support transportation agencies in evaluating and measuring performance of surface pavement over time for the long-term 
rehabilitation and maintenance programming. This study adopted the models from the past literature, Irfan (2010), to evaluate the impact of rehabilitation treatment on two main indicators, performance jump and post-treatment performance trend. Figure 5 demonstrates how performance jump and the post-treatment performance trend are considered over the long-term pavement performance curve. Therefore, the IRI of pavement at any year over an analysis period can be mainly evaluated based on the initial conditions of the pavement, selected treatment method, age of pavement as well as performance jump and posttreatment performance. The detailed description of this module can be reclaimed from chapter 4.

\subsection{3 $\mathrm{CO}_{2}$ Emission Estimating Module}

This module is adopted from the network energy consumption estimating model (as mentioned in chapter 4) to evaluate and measure the impact of rehabilitation treatment implementation on $\mathrm{CO}_{2}$ emissions throughout the transportation network. As mentioned in Figure 5, two main components are focused in this module: (1) $\mathrm{CO}_{2}$ emissions during the construction operations; and (2) $\mathrm{CO}_{2}$ emissions under the regular operation of a road. First, under construction, travelers are induced to reduce their speeds while traveling through work-zone. This reduction in average traveling speed is the result of a regulated speed limit and traffic congestion as the traffic volume is accumulated due to less road capacity from the lane closure. This impact of rehabilitation efforts will remain until the construction is completed and traffic is resumed to the regular operation, with an increase in the fuel consumption rate of vehicles and therefore total $\mathrm{CO}_{2}$ emissions in the network. Second, after the completion of the rehabilitation activity, the surface pavement will have better conditions. This improvement in road conditions will significantly lead to a variation in 
energy consumption and $\mathrm{CO}_{2}$ emissions. The impact can be conglomerated in the aspect of transportation networks. The mathematical equations for calculating these two types of impact are given in Figure 5. $\mathrm{CO}_{2}$ emissions are calculated by multiplying energy consumption with an emission factor of an associated fuel type consumed in vehicles. This emission factor represents the amount of $\mathrm{CO} 2$ emissions per one gallon or liter of fossil fuel and it can have different values based on the type of fuel used. In this study, the emission factor is determined as 2.325 and $2.668 \mathrm{~kg} /$ liter (8.8 and $10.1 \mathrm{~kg} /$ gallon) for gasoline and diesel fuel, respectively, according to the EPA (2005).

\subsubsection{Travel-Delay Cost Estimating Module}

During the construction operations, travelers experience traffic delay from the reduction in traveling speed due to: (1) the work-zone speed limit enforced by the government regulations to control safety under the construction zone; (2) an accumulated traffic volume affected from a lower capacity of the road due to the lane closure; and (3) an increase of traffic volume on detour routes as a result of traffic diversions to avoid the slow speed on the constructing route. In this study, the travel-delay cost estimating module is developed to measure an increase in travel cost expected from travel delay during the construction operations as a result of rehabilitation treatment implementation. The traveldelay cost can be simply estimated by using the equation as shown in Figure 5. In addition to the above description, the development of the module and its calculation algorithm can be further referred from chapter 4 .

\subsubsection{Road User Cost Savings Estimating Module}

This module was developed to facilitate decision makers in estimating the expected savings in road user costs resulting from an application of rehabilitation efforts on 
transportation networks. The calculation will be performed over an analysis period to measure the impact of highway decision making on the long-term road user cost savings. In this study, the main components in road user costs are considered: (1) fuel consumption cost, (2) tire depreciation cost and (3) vehicle repair and maintenance costs. The calculation concept is related to better surface conditions of the pavement after rehabilitation that leads to a decrease in road user costs. The savings of these three cost components will be measured by estimating their changes before and after the implementation of rehabilitation treatment. Figure 5 provides the mathematical equations necessary for the estimation. The further information associated to the development of this module can be also adopted from chapter 4.

\subsection{Optimization Constraints}

This section describes the constraints used in the developed multi-objective optimization model. As aforementioned, the analysis will be performed by taking into consideration the budget constraints from the transportation planning agencies' perspectives. The available funding will be predetermined for an annual budget, a 5-year rehabilitation program and a 20-year highway rehabilitation plan. In this study, there are four 5-year rehabilitation programs over a 20-year analysis lifespan. It is also assumed that decision makers will make decisions relevant to three types of decision variables mentioned earlier in the previous section - road selection, treatment timing, and treatment method, once at the beginning of each of the four rehabilitation program cycles. 


\subsection{Model Evaluation}

In this section, the developed multi-objective optimization model was tested with two case studies in order to evaluate its performance and capabilities in planning and optimizing highway rehabilitation efforts. The first case study aims to optimize the highway rehabilitation efforts of the hypothetical road network in Miami-Dade County, Florida. The second example seeks to demonstrate the model's capabilities in planning and optimizing the larger transportation networks covering the area of Lake City District (District 2), Florida. The detailed analysis of each example is presented in the following subsections.

\subsubsection{Example 1: Hypothetical road network in Miami-Dade County}

This example represents the capabilities of the developed model in planning and optimizing highway rehabilitation efforts on the aging road network. The case study is composed of ten road sections randomly selected from the different locations throughout the real transportation network in Miami-Dade County, Florida. All ten road sections are hypothetically assumed to be suffering and need rehabilitation due to pavement deterioration. The model was adopted to plan and optimize all ten candidate projects under budget constraints. Table 5-1 illustrates the data of all ten candidate road sections. The data include: (1) current conditions of pavement in terms of IRI; (2) total traffic volume in terms of annual average daily traffic (AADT); (3) length of road section; (4) fee-flow speed; (5) work-zone speed; (6) number of lane in each direction; and (7) total equivalent standard axle load for each road section. 
Table 5-1 Candidate rehabilitation projects in Example 1

\begin{tabular}{|c|c|c|c|c|c|c|c|}
\hline Project & $\begin{array}{c}\text { IRI } \\
\text { (m/km) }\end{array}$ & $\begin{array}{c}\text { Total } \\
\text { traffic } \\
\text { volume } \\
\text { (veh/day) }\end{array}$ & $\begin{array}{c}\text { Length } \\
\text { (mile) }\end{array}$ & $\begin{array}{c}\text { Free- } \\
\text { flow } \\
\text { speed } \\
\text { (mph) }\end{array}$ & $\begin{array}{c}\text { Work } \\
\text { zone } \\
\text { speed } \\
\text { (mph) }\end{array}$ & $\begin{array}{c}\text { Number } \\
\text { of lanes }\end{array}$ & $\begin{array}{c}\text { Total ESAL } \\
\text { (x106) } \\
\text { (ESAL/lane) }\end{array}$ \\
\hline 1 & 4.5 & 45,500 & 2.87 & 40 & 25 & 4 & 0.3546 \\
2 & 3.2 & 55,000 & 2.11 & 40 & 25 & 3 & 0.5715 \\
3 & 2.8 & 37,500 & 4.05 & 40 & 25 & 2 & 0.5845 \\
4 & 3 & 50,500 & 2.00 & 45 & 30 & 3 & 0.5247 \\
5 & 4 & 35,000 & 2.04 & 35 & 20 & 2 & 0.5455 \\
6 & 4 & 48,500 & 1.62 & 40 & 25 & 3 & 0.5039 \\
7 & 3.8 & 33,500 & 1.69 & 45 & 30 & 3 & 0.3481 \\
8 & 5 & 63,000 & 2.66 & 45 & 30 & 3 & 0.6546 \\
9 & 4 & 13,000 & 1.74 & 40 & 25 & 1 & 0.4052 \\
10 & 3.8 & 71,000 & 2.24 & 45 & 30 & 3 & 0.7377 \\
\hline
\end{tabular}

As aforementioned, an analysis was performed under a 20-year lifespan period consisting of four 5-year highway rehabilitation programs. The total available funding for a 20-year plan and 5-year program are assumed to be $\$ 60$ and $\$ 15$ million, respectively. The decision making is assumed to be made by decision makers in order to select which road sections will be implemented (RS), timing when the treatment will be applied (TT), and the type of treatment (TM), at the beginning of each of the rehabilitation program cycles.

The truck volume is assumed as $10 \%$ of AADT on each road section. The unit cost of travel-delay is determined with $\$ 23$ per vehicle-hour, according to Copeland (1998). All costs and benefits are calculated at a 5\% discount rate net present value with the adjustment of the customer price index (Statistics 2014) for the analysis. The rehabilitation cost per lane-mile for specific treatment alternatives used in this study are estimated by using an average value from Irfan (2010), as shown in Table 5-2. The rehabilitation durations are 
similarly estimated by referring the data from Caltrans (2015) and OECD (2005), as shown in Table 5-3. The total cost and duration of each road section will be varied based on the type of rehabilitation treatment alternative applied on the pavement.

Table 5-2 Average unit cost (in 2015 U.S. dollars/lane-km) for rehabilitation treatment alternatives (modified from Irfan 2010)

\begin{tabular}{|c|c|}
\hline Treatment Alternative & $\begin{array}{c}\text { Unit Cost (year 2015) } \\
\mathbf{( \$ / l a n e - k m ) ~}\end{array}$ \\
\hline Thin HMA overlay & 55,454 \\
Functional HMA overlay & 71,119 \\
Structural HMA overlay & 104,720 \\
Full depth HMA & 634,275 \\
\hline
\end{tabular}

Table 5-3 Average construction duration (day/lane-km) for rehabilitation treatment alternatives (modified from Caltrans 2015; OECD 2005)

\begin{tabular}{|c|c|}
\hline Treatment Alternative & $\begin{array}{c}\text { Construction Duration } \\
\text { (day/lane-km) }\end{array}$ \\
\hline Thin HMA overlay & 1.25 \\
Functional HMA overlay & 2.5 \\
Structural HMA overlay & 5 \\
Full depth HMA & 9.5 \\
\hline
\end{tabular}

The result demonstrates the model's capabilities in generating an equally-optimal highway rehabilitation programs. The set of solutions represents an optimal and non-dominated relationship between the two planning objectives: (1) minimizing environmental impact in terms of $\mathrm{CO}_{2}$ emissions; and (2) maximizing net public benefits, with 50 near equally-optimal highway rehabilitation programs generated, as shown in Figure 5-6. The set of optimal solutions tends to generate lower environmental impact in transportation networks with 2,756 million kilograms or $0.00035 \mathrm{ppm}$ of $\mathrm{CO}_{2}$ averagely for Example 1. Average vehicle approximately creates $0.22 \mathrm{~kg}$ of $\mathrm{CO}_{2}$ per vehicle per lane- 
mile. Additionally, the result shows that maximizing net public benefits of highway rehabilitation efforts can lead to an increase in $\mathrm{CO}_{2}$ emissions in transportation networks.

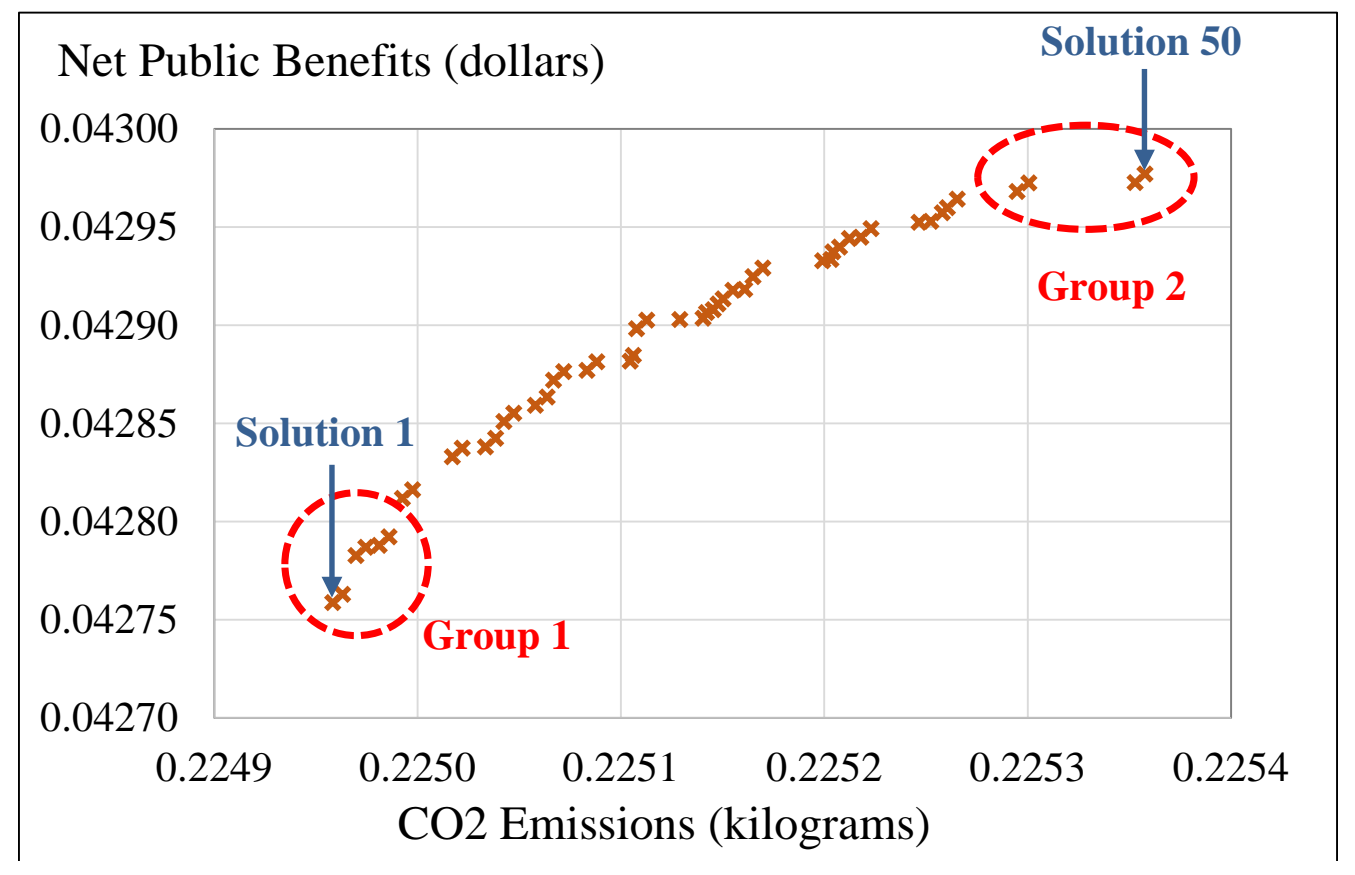

Figure 5-6 Relationship between net public benefits and $\mathrm{CO}_{2}$ emissions per vehicle per lane-mile in Example 1

A closer examination was performed by focusing on some solutions and categorizing them into two groups, as indicated in Figure 5-6. Group 1 represents the highway rehabilitation programs with the lowest values of $\mathrm{CO}_{2}$ emissions and net public benefits, and vice versa for Group 2. The result revealed that the road selection and treatment timing variables were likely to have the same value for all possible highway programs. The differences, however, were in the variable related to the treatment method. The rehabilitation programs in Group 1 that minimize $\mathrm{CO}_{2}$ emissions tend to select more expensive treatment methods compared to Group 2, which maximizes net public benefits. This trend continues in each of the four rehabilitation program cycles over a 20-year planning period. A further analysis on pavement conditions of all road sections and the 
transportation network on both groups were examined. Figure 5-7 shows that the highway rehabilitation programs in Group 1 that spend more money tend to improve overall performance of the road network better than the less expensive programs in Group 2. This confirms the past studies about an inverse relationship between rehabilitation costs and pavement performance (Fwa and Sinha 1991), which stated that an advanced construction quality and techniques typically require a high level of financial resources to have better pavement performance (Wang et al. 2003). In addition, the overall network performance tends to improve over the analysis period as a result of rehabilitation implementation in every program cycle. However, it is worth noting that all possible solutions generated in this example provide the overall network pavement performance in a good condition with the average IRI value lower than $2.9 \mathrm{~m} / \mathrm{km}$ (180 in/mile).

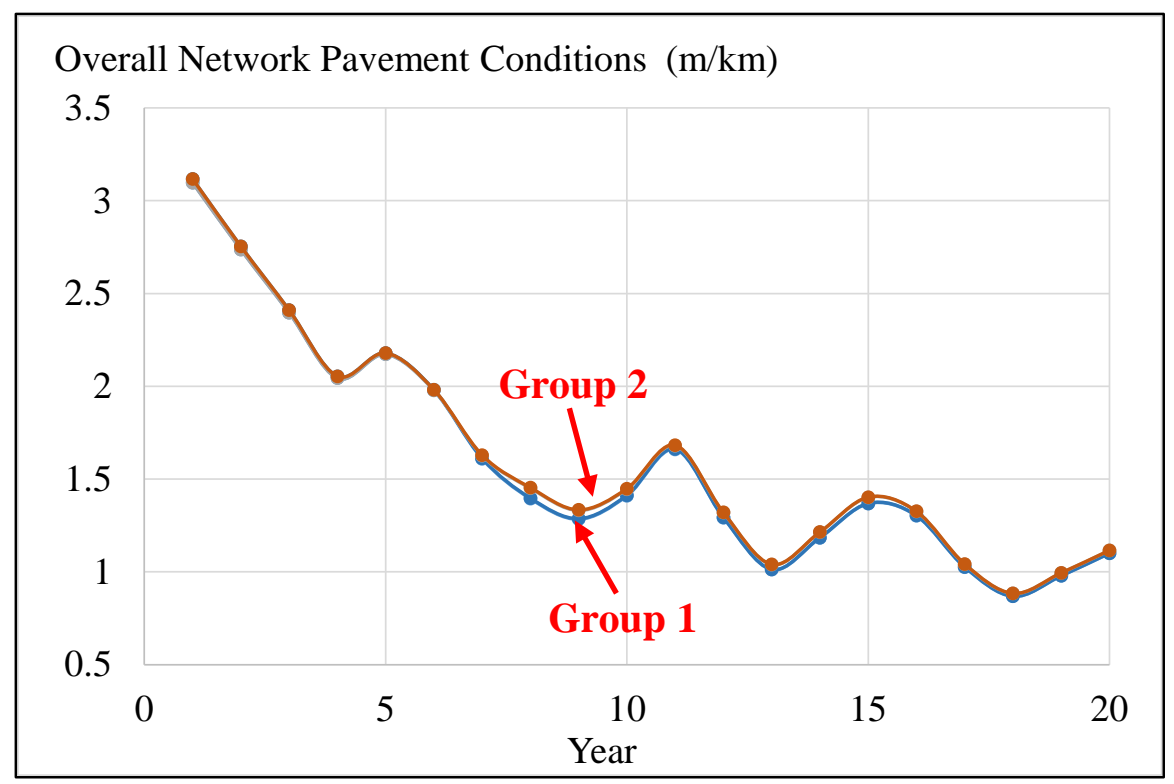

Figure 5-7 Overall network pavement conditions over the planning period in Example 1

Considering the road level, the average pavement conditions of all road sections over a 20-year analysis period are a good or very good level, as shown in Figure 5-8. Two 
highway rehabilitation programs (Solution 1 and 50) were compared with the bar chart, showing worse conditions (higher IRI value) from Solution 50. However, it should be noted that the pavement conditions shown in Figure 5-8 were calculated based on the IRI value averaged over the analysis lifespan on the road. Therefore, the road sections may have a high level of IRI and range worse beyond fair conditions in some circumstances.

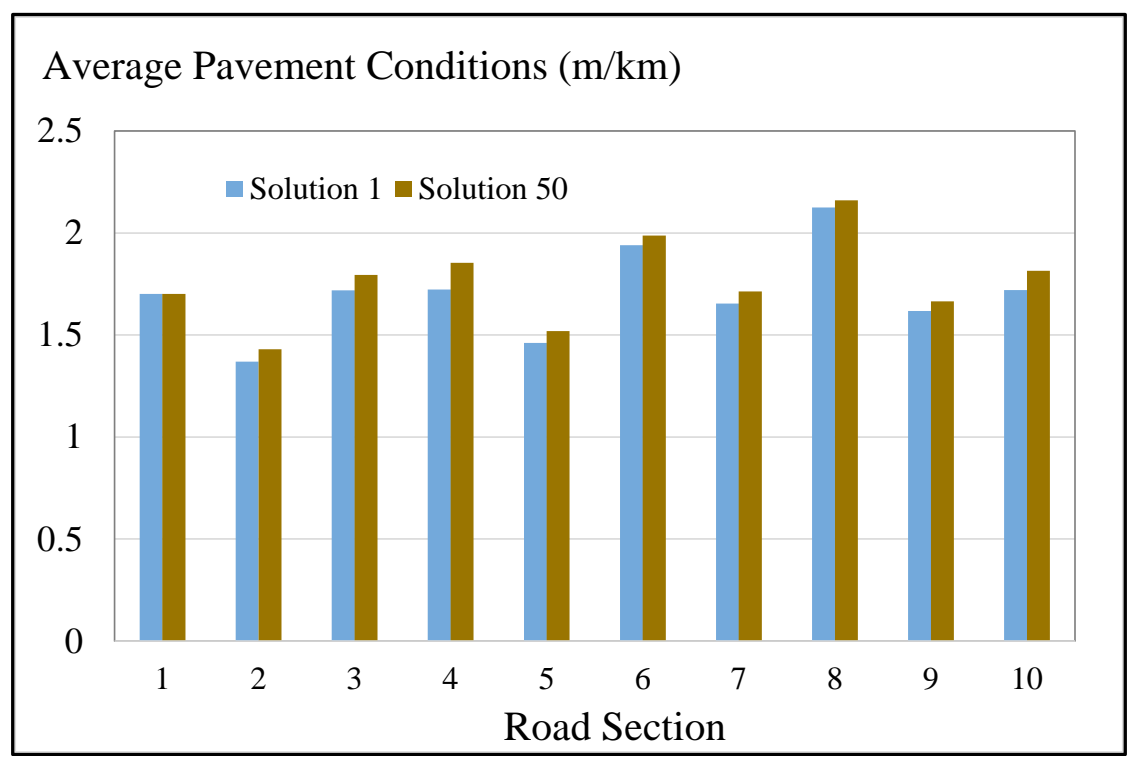

Figure 5-8 Comparison of average pavement conditions for each road section

The further investigation was performed to receive more perception of how rehabilitation cost spent on the highway rehabilitation program affects public benefits and $\mathrm{CO}_{2}$ emissions over the transportation network. Figure 5-9 illustrates the relationships between public benefits and rehabilitation cost, and $\mathrm{CO}_{2}$ emissions and rehabilitation cost, respectively. It shows that increasing rehabilitation cost tends to generate a higher public benefits in terms of road user cost savings and vice versa for network $\mathrm{CO}_{2}$ emissions. However, when considering in terms of net public benefits that are calculated as the balance between road user cost savings and rehabilitation cost, lower rehabilitation cost is likely to 
provide higher $\mathrm{CO}_{2}$ emissions and net public benefits. This tradeoff relationship can be supported with the closer examination of the ratio of public benefits to one dollar of rehabilitation cost, and the expected cost per one $\mathrm{kg}$ of $\mathrm{CO}_{2}$ emissions respectively, between two groups of highway rehabilitation programs, as shown in Figure 5-10.

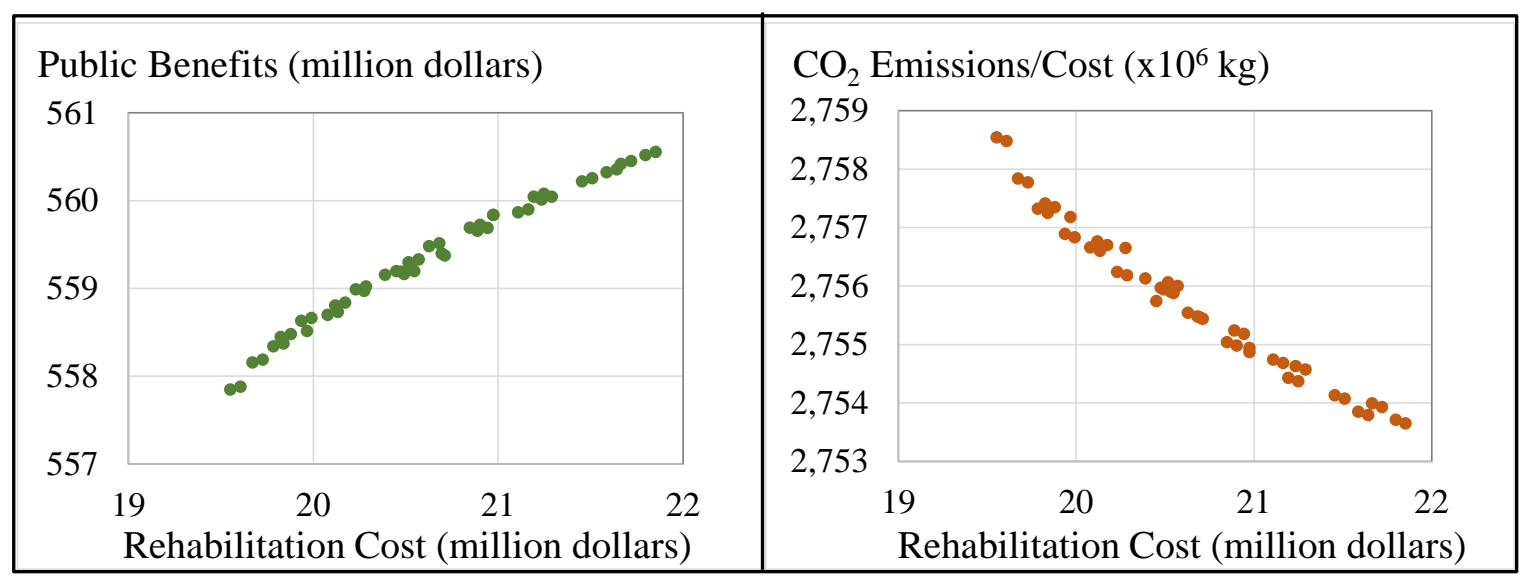

Figure 5-9 Relationships between rehabilitation cost and (a) public benefits (b) $\mathrm{CO}_{2}$ emissions in Example 1

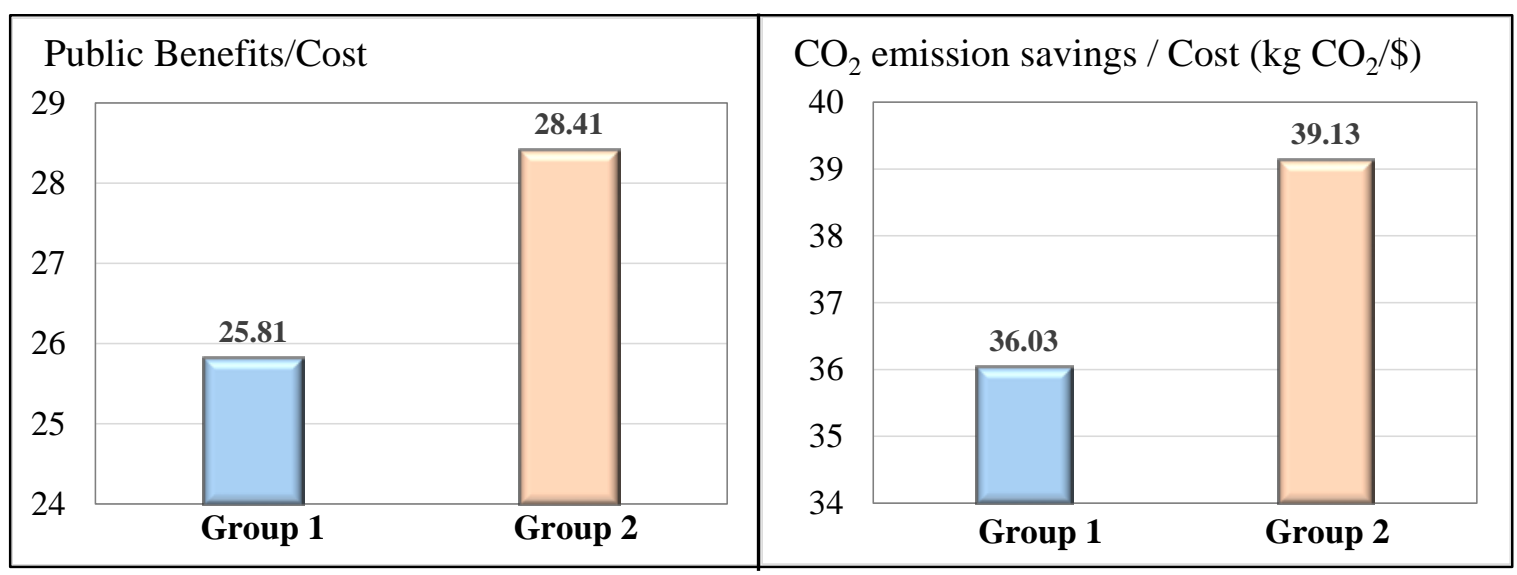

Figure 5-10 Comparison of public benefits/cost and $\mathrm{CO}_{2}$ emission savings/cost between two groups of rehabilitation programs in Example 1

The ratio of public benefits to rehabilitation cost is higher when applying a less expensive treatment on pavement (see Group 2). Therefore, at the same rehabilitation cost, using a more expensive treatment (Group 1) tends to generate lower net public benefits. It 
can also lead to a reduction in $\mathrm{CO}_{2}$ emissions throughout the transportation network as a result of better pavement conditions from a higher-cost and more effective rehabilitation treatment alternative.

\subsubsection{Example 2: Road network in Lake City District, Florida.}

In this section, an application example of a transportation network covering District 2 in Florida was analyzed to demonstrate the model performance and capabilities when implemented to a larger transportation network. The example is composed of 27 road sections that are suffering and need rehabilitation due to pavement deterioration at different locations throughout the transportation network. Table 5-4 presents the data necessary for an analysis of all road sections, including: (1) length of road section; (2) total traffic volume in terms of annual average daily traffic (AADT); (3) total traffic truck volume in terms of annual truck traffic volume (AATA); (4) current conditions of pavement in terms of IRI; (5) fee-flow speed; (6) work-zone speed; and (7) number of lane in each direction.

The similar data in example 1 was applied for calculating a rehabilitation cost and duration of a specific treatment alternative. The total available budget is determined as \$200 and \$800 million for a 5-year rehabilitation program and a 20-year highway rehabilitation plan, respectively, to optimize rehabilitation efforts at the beginning of each of the four 5-year program cycles.

The result demonstrates the relationship between $\mathrm{CO}_{2}$ emissions and net public benefits, as shown in Figure 5-11, with a range of 44 equally-optimal highway rehabilitation programs. This trend also shows that minimizing $\mathrm{CO}_{2}$ emissions in the transportation network can cause a lowering in net public benefits. The result also shows a 
Table 5-4 Candidate rehabilitation projects in Example 2

\begin{tabular}{|c|c|c|c|c|c|c|c|}
\hline $\begin{array}{l}\text { Road } \\
\text { section }\end{array}$ & $\begin{array}{l}\text { Length } \\
(\mathrm{km})\end{array}$ & $\begin{array}{c}\text { Traffic } \\
\text { volume } \\
\text { (veh/day) }\end{array}$ & $\begin{array}{c}\text { Truck } \\
\text { volume } \\
\text { (veh/day) }\end{array}$ & $\begin{array}{c}\text { IRI } \\
(\mathrm{m} / \mathrm{km})\end{array}$ & $\begin{array}{l}\text { free-flow } \\
\text { speed } \\
(\mathrm{km} / \mathrm{h})\end{array}$ & $\begin{array}{c}\text { Work-zone } \\
\text { speed } \\
(\mathrm{km} / \mathrm{h})\end{array}$ & $\begin{array}{l}\text { Number } \\
\text { of lanes }\end{array}$ \\
\hline 1 & 3.4 & 2,300 & 115 & 3.5 & 72.4 & 56.3 & 2 \\
\hline 2 & 55.9 & 73,000 & 5,329 & 3.5 & 104.6 & 80.5 & 3 \\
\hline 3 & 56.6 & 56,000 & 10,472 & 4.5 & 112.7 & 88.5 & 3 \\
\hline 4 & 5.9 & 33,500 & 7,906 & 4 & 112.7 & 88.5 & 3 \\
\hline 5 & 53.1 & 21,500 & 6,300 & 4 & 112.7 & 88.5 & 2 \\
\hline 6 & 49.0 & 33,500 & 7,906 & 4 & 112.7 & 88.5 & 3 \\
\hline 7 & 7.9 & 15,900 & 3,800 & 3.5 & 72.4 & 56.3 & 2 \\
\hline 8 & 41.1 & 26,346 & 5,743 & 4 & 112.7 & 88.5 & 2 \\
\hline 9 & 33.3 & 20,540 & 4,991 & 4 & 112.7 & 88.5 & 2 \\
\hline 10 & 46.3 & 34,000 & 8,568 & 3.5 & 112.7 & 88.5 & 3 \\
\hline 11 & 40.9 & 20,000 & 6,780 & 3.5 & 112.7 & 88.5 & 2 \\
\hline 12 & 1.9 & 11,400 & 1,756 & 3.5 & 56.3 & 40.2 & 2 \\
\hline 13 & 3.7 & 48,000 & 912 & 3.5 & 88.5 & 72.4 & 2 \\
\hline 14 & 10.3 & 31,000 & 651 & 4.5 & 48.3 & 32.2 & 2 \\
\hline 15 & 16.9 & 60,000 & 10,260 & 4 & 112.7 & 88.5 & 3 \\
\hline 16 & 2.8 & 11,000 & 924 & 4 & 56.3 & 40.2 & 2 \\
\hline 17 & 17.0 & 79,500 & 6,281 & 3.5 & 88.5 & 72.4 & 4 \\
\hline 18 & 5.6 & 27,000 & 567 & 4 & 56.3 & 40.2 & 3 \\
\hline 19 & 41.0 & 58,500 & 8,015 & 4 & 104.6 & 80.5 & 2 \\
\hline 20 & 27.1 & 116,500 & 8,505 & 4 & 104.6 & 80.5 & 3 \\
\hline 21 & 11.8 & 14,000 & 3,948 & 4 & 96.6 & 72.4 & 2 \\
\hline 22 & 0.9 & 43,000 & 5,891 & 4 & 104.6 & 80.5 & 3 \\
\hline 23 & 1.1 & 20,000 & 6,780 & 3.5 & 112.7 & 88.5 & 2 \\
\hline 24 & 19.7 & 60,000 & 10,260 & 4.5 & 112.7 & 88.5 & 3 \\
\hline 25 & 56.1 & 81,000 & 9,801 & 3.5 & 112.7 & 88.5 & 3 \\
\hline 26 & 34.5 & 107,000 & 5,243 & 3 & 72.4 & 56.3 & 3 \\
\hline 27 & 8.9 & 16,300 & 4,597 & 3.5 & 104.6 & 80.5 & 2 \\
\hline
\end{tabular}

lower $\mathrm{CO}_{2}$ emissions from the generated solutions with 74.28 billion kilograms or 0.00951 ppm of $\mathrm{CO}_{2}$ averagely in this example. An average $\mathrm{CO}_{2}$ emission is calculated as $0.16 \mathrm{~kg}$ of $\mathrm{CO}_{2}$ per vehicle per lane-mile. From a closer analysis of possible solutions in this example, it shows no difference in assigning the road selection variable between Solution 
1 that minimize $\mathrm{CO}_{2}$ emissions and Solution 44 that attempt to maximize net public benefits. However, the differences were in the other two variables, which are about treatment timing and treatment method.

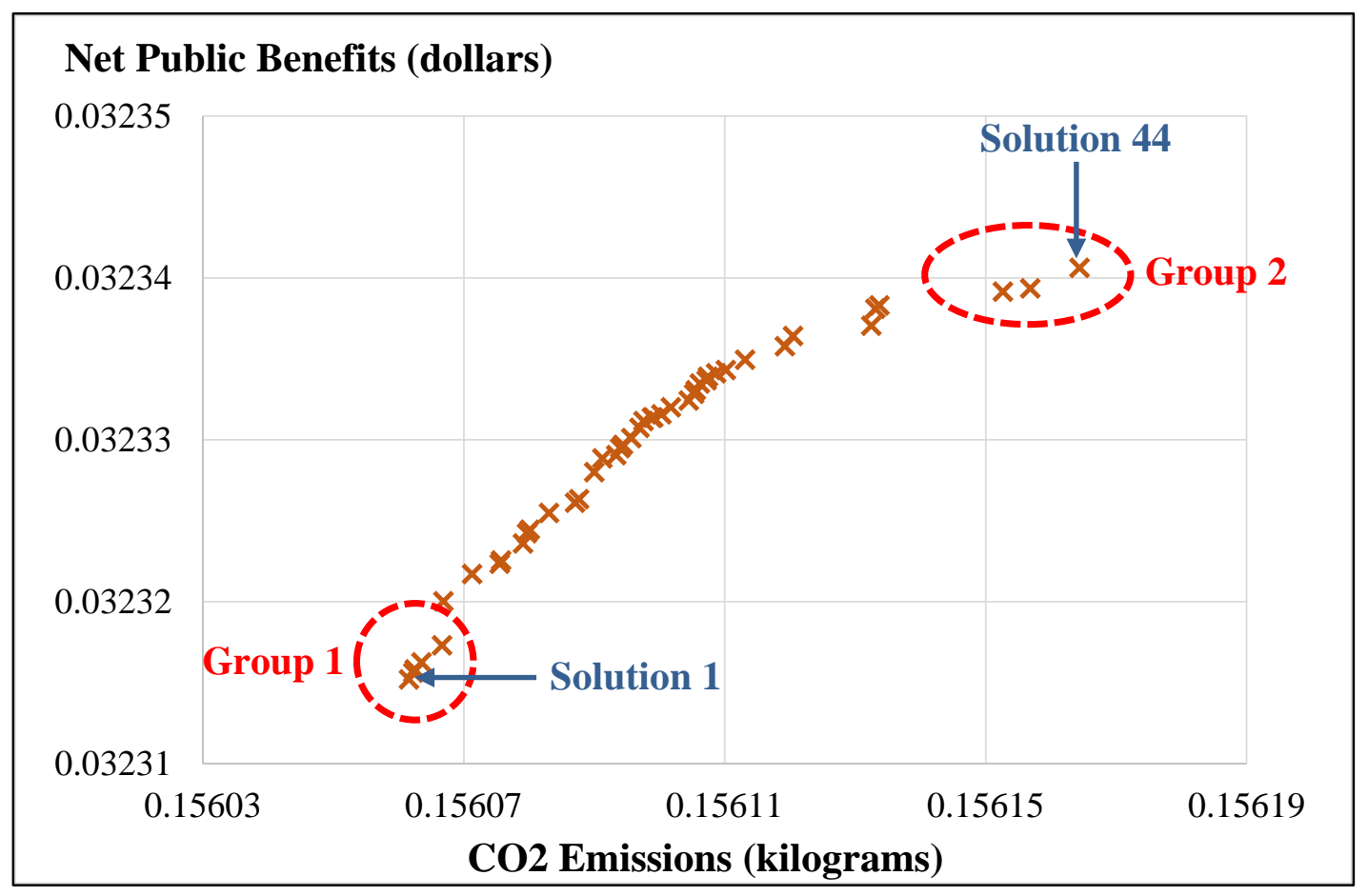

Figure 5-11 Relationship between net public benefits and $\mathrm{CO}_{2}$ emissions per vehicle per lane-mile in Example 2

Considering the decision variables in detail, Solution 44 tends to select a less expensive treatment method compared to Solution 1. However, a less expensive program does not guarantee the early-treatment application in this example. The analysis shows no pattern in selecting the year of implementation between these two solutions. This may result from several factors, such as the conditions of pavement at the time of application, the possible treatment methods, and the remaining financial budget.

The further examination was focused on two groups of solutions, as shown in Figure 5-11. Group 1 represents the highway programs with low $\mathrm{CO}_{2}$ emissions and net 
public benefits, and vice versa for Group 2. Considering the total rehabilitation cost spent on the program, Group 1 tends to spend more money in repairing the road network. This results in better average conditions of pavement in the transportation network, as shown in Figure 5-12. The overall network conditions tend to be improved over the analysis lifespan with some variations due to the road usage and highway rehabilitation decisions. All solutions along the tradeoff provides the good conditions of the network pavement in the average IRI value of less than $2.9 \mathrm{~m} / \mathrm{km}$. It is also noteworthy that a small difference in IRI between these two groups can lead to a very significant impact in public benefits and $\mathrm{CO}_{2}$ emissions when taking into account some road characteristics, such as traffic volume, length of the road in the network, and number of lanes on each road.

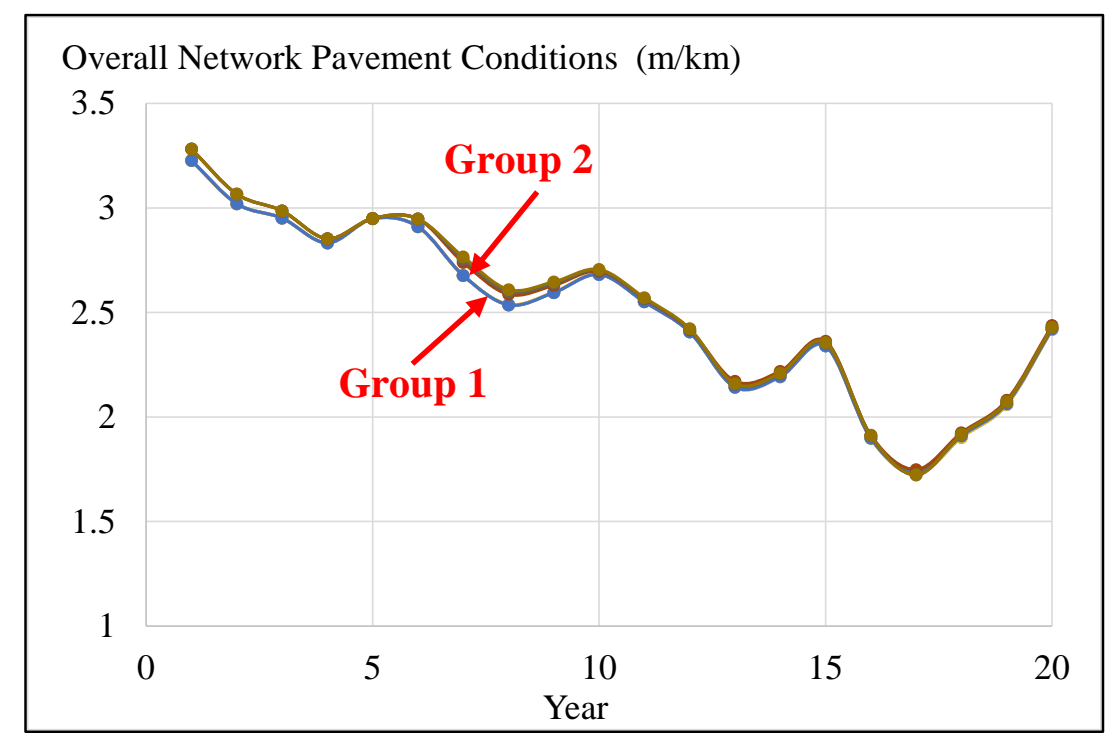

Figure 5-12 Overall network pavement conditions over the planning period in Example 2

The relationships between public benefits and rehabilitation cost, and $\mathrm{CO}_{2}$ emissions and rehabilitation cost, in this example, are additionally demonstrated in Figure 5-13. The result shows similar trends as in Example 1, with an increase in public benefits and a reduction in $\mathrm{CO}_{2}$ emissions when investing more money in the network 
rehabilitation. Moreover, the benefit/cost $(\mathrm{B} / \mathrm{C})$ ratio and the ratio of cost per one $\mathrm{kg}$ of $\mathrm{CO}_{2}$ emissions were calculated and compared between Group 1 and 2, as illustrated in Figure 5-14. The result confirms that a less expensive rehabilitation program (Group 2) tends to generate higher net public benefits as a result of a larger $\mathrm{B} / \mathrm{C}$ ratio. Assuming one dollar spent in rehabilitating the road network, Group 2 will provide $\$ 39.56$ in public benefits, which is almost $\$ 3$ higher than Group 1.

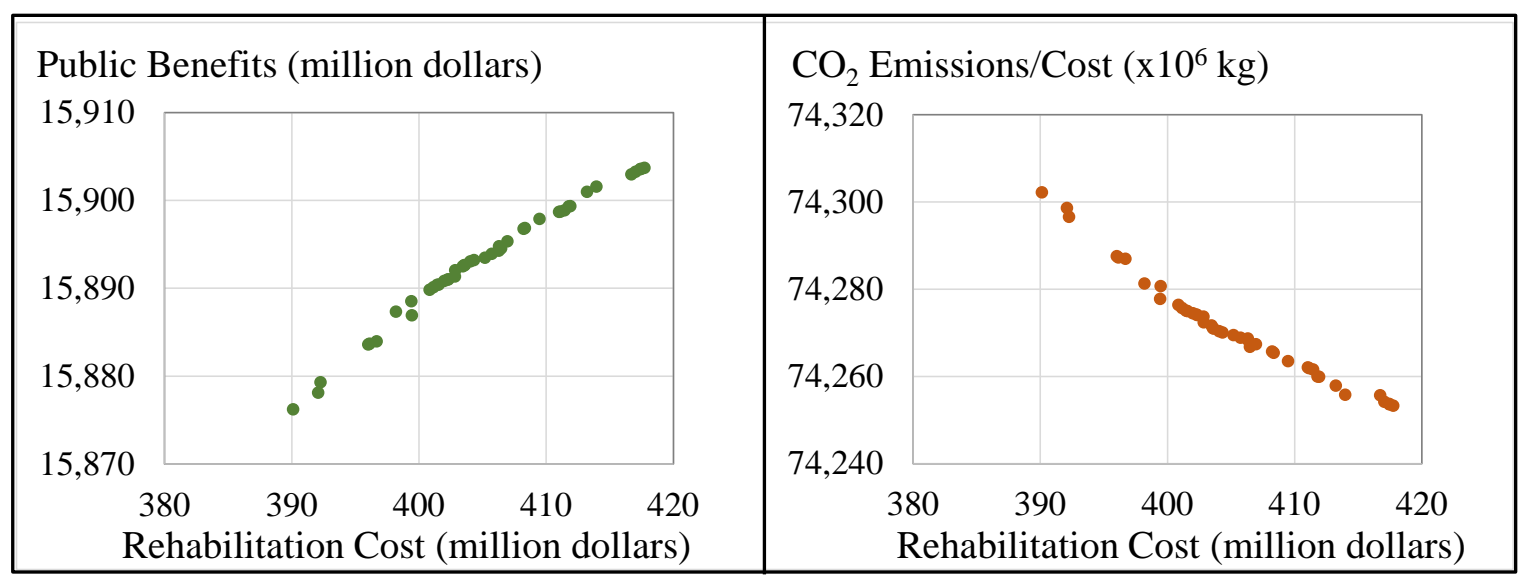

Figure 5-13 Relationships between rehabilitation cost and (a) public benefits (b) $\mathrm{CO}_{2}$ emissions in Example 2

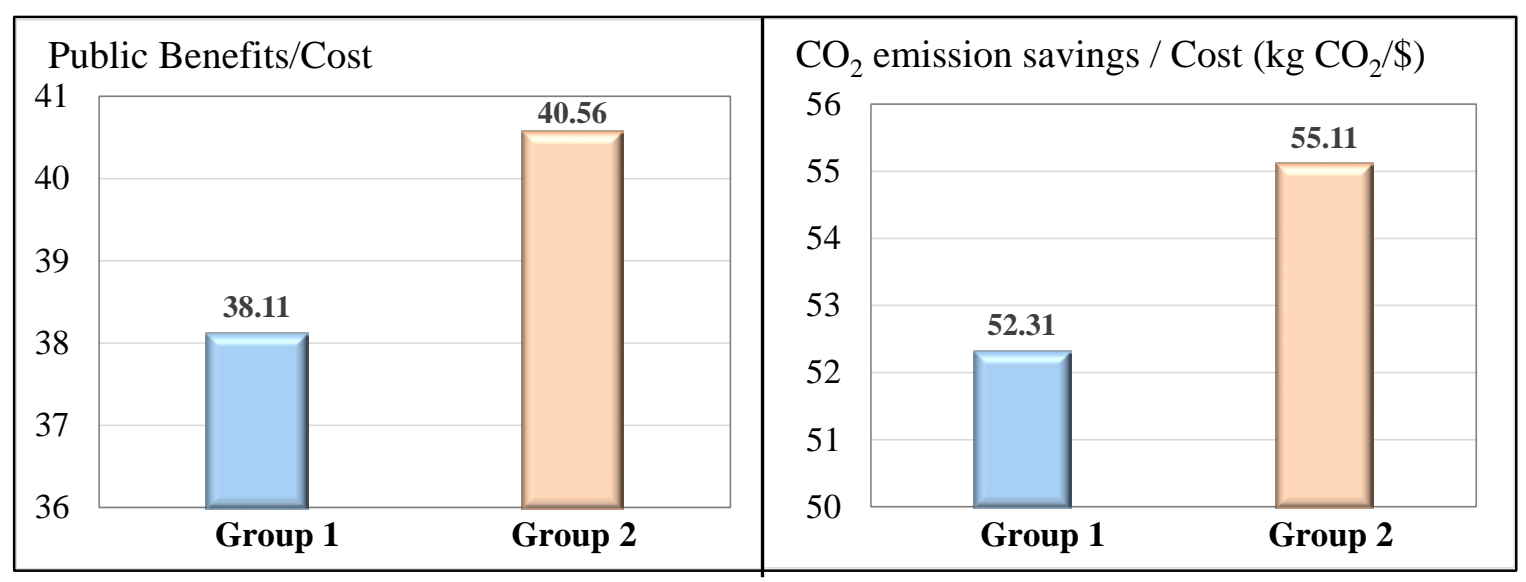

Figure 5-14 Comparison of public benefits/cost and $\mathrm{CO}_{2}$ emission savings/cost between two groups of rehabilitation programs in Example 2 
The unit cost per one $\mathrm{kg}$ of $\mathrm{CO}_{2}$ emissions also has a similar finding as the transportation network in Example 1. The ratio in Group 1 is higher than Group 2, which means, to decrease one unit of $\mathrm{CO}_{2}$ emitted to environment, Group 1 spends a little more money when compared to Group 2. This may result from the difference in selecting the treatment of these two groups. Group1 tends to select a higher cost treatment to improve the pavement conditions to good or very good conditions, while Group 2 differently selects a lower cost treatment to reach a good condition of pavement improvement.

The two examples analyzed in this section confirm the application and capabilities of the developed model in searching for and identifying optimal or near optimal rehabilitation programs. It provides decision makers with a wide set of the tradeoff solutions between the environmental impact in terms of $\mathrm{CO}_{2}$ emissions and net public benefits. This model should prove useful to transportation planning agencies in promoting sustainability and concurrently addressing public and agency perspectives in highway rehabilitation efforts.

\subsection{Feedback from Department of Transportation}

In this section, the personnel in the Department of Transportation who are involved in the programming and planning processes of the highway rehabilitation investment were asked to participate in a discussion panel and provide their feedback regarding the different main aspects of the model development. The main objective of this interview is to validate an appropriateness and applicability of the developed model in facilitating decision making of transportation planners and promoting the environmental sustainability in current highway rehabilitation efforts. The summary of feedback and comments was provided as follows. 


\subsubsection{Appropriateness of decision variables}

$>$ In the current practice of the highway rehabilitation, planners and decision makers make decisions by selecting the projects from the list of candidate road sections for each year of implementation along a 5-year program. The potential treatment methods are also assigned primarily based on pavement condition. In addition, there are other factors, such as pavement age, traffic volume, and truck volume, included and weighed into the rehabilitation programming.

The developed model included the types of decisions that are typically considered by transportation planners in highway programming.

\subsubsection{Appropriateness of optimization constraints}

The Department of Transportation considers two constraints in the current practice, which are the availability of budget and the minimum requirement for overall pavement conditions throughout the transportation network.

The developed model was constructed by considering the budget availability as one of the optimization constraints.

$>$ The constraint for the overall network pavement condition was constructed with a few differences from the current practice used in the participating transportation agency because of the difference of the pavement indicator used. The study adopted the common indicator, IRI, for the model and analysis, while the participating agency currently used the Pavement Condition Survey that considers three main parameters - rutting, cracking, and ride quality for evaluating pavement performance. This creates a challenge in applying the same constraints as used in the current practice in the model development. Therefore, the model was constructed by including the pavement conditions for the treatment selection to 
be improved to at least good conditions. The overall network conditions were checked from the optimization results, which always reach a good or very good condition. However, it is worth noting that the participants mentioned the high possibility in using IRI as the pavement performance indicator in their agency in the near future.

\subsubsection{Appropriateness of optimization planning objectives}

The participating Department of Transportation does not apply an optimization in the current highway rehabilitation practice. The rehabilitation programs will be primarily considered based on the pavement conditions. The candidate road sections will be ranked as the pavement with worst conditions will be considered in the top priority. The annual budget will be allocated to the candidate projects. With the limited funding, some of the next-priority candidate projects will be deferred and use the budget of the following years. However, the transportation planners may ask for additional budget from the relevant divisions in case that more rehabilitation is needed to meet the requirement of overall network pavement conditions.

The developed model was constructed by considering the environmental sustainability and net public benefits as the planning objectives. The environmental sustainability was included in the model development to demonstrate the model capabilities in integrating the sustainability in the current highway rehabilitation practice. Moreover, net public benefit is always taken into account in prioritizing resurfacing and rehabilitation projects. The technical report shows transportation planners' concern in maximizing the benefits of road surfacing by considering the annual user benefit of driver and average traffic volume. 


\subsubsection{Current implementation of environmental sustainability}

$>$ The current practice of the Department of Transportation does not specifically include the environmental sustainability in the highway rehabilitation programming. The environmental assessment is currently performed by the environmental management office and it is mainly focused on the "capacity-related" projects, such as lane widening, or the "transportation-mode-changing” projects, such as bicycle way construction. However, the environmental performance is currently measured at the regional level, which consolidates several nearby counties into a single metropolitan planning organization (MPO) and generates coordinated planning efforts.

The environmental assessment of the Department of Transportation is currently focused on several aspects, including the historical aspect, natural aspect, air quality, water quality and contamination, etc. This means the air quality in terms of $\mathrm{CO}_{2}$ emissions is presently one of the potential indicators in measuring the environmental sustainability of highway efforts.

5.7.5 Applicability of the model to support sustainability in highway rehabilitation efforts

The model was presented to the Department of Transportation personnel by showing the model framework and outcome. The participants were asked about the feasibility and applicability of the developed model in being a starting point to support the sustainability in the highway rehabilitation programming.

There is a high feasibility and applicability to integrate the environmental sustainability in highway rehabilitation efforts. The proposed model in this study can facilitate and be useful for the future implementation. 


\subsection{Sensitivity Analysis}

Sensitivity analysis was performed to determine how the uncertainty of an independent variable used in the developed model will impact an analysis result. Therefore, in this study, the sensitivity was measured based on a variability of the sets of optimal highway programs generated from the environmentally-conscious decision-support model. The effects from three groups of input variables were considered in this section: (1) initial pavement conditions; (2) budget availability; and (3) some parameters that were applied with the assumptions in the development of the vehicle fuel consumption estimating module in Chapter 3. The results and their interpretation are given as follows.

\subsubsection{Effect of initial pavement conditions}

The impact of initial pavement conditions (in terms of IRI) was investigated by varying the IRI values from $-20 \%$ (better pavement conditions) to $+20 \%$ (worse conditions), as a smaller IRI means a better pavement condition. All sets of optimal highway programs generated in this analysis are shown in Figure 5-15.

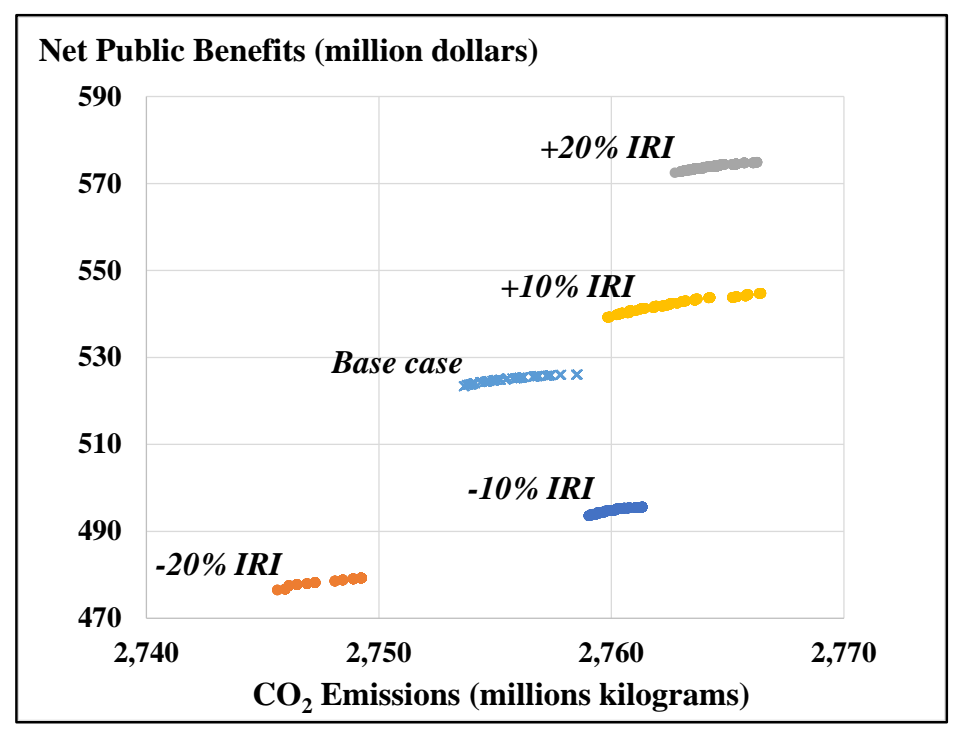

Figure 5-15 Sensitivity analysis from an uncertainty in initial pavement conditions 
It can be concluded that the uncertainty in an initial IRI has an impact on the optimal solutions. The model tends to generate the set of optimal solutions that have lower $\mathrm{CO}_{2}$ emissions and net public benefits if the transportation network is covered with bettercondition pavements. From the analysis, it shows a variation between $-0.3 \%$ to $0.33 \%$ in $\mathrm{CO}_{2}$ emissions and $-9 \%$ to $9.4 \%$ in net public benefits when changing IRI between $-20 \%$ to $+20 \%$.

\subsubsection{Effect of a 5-year program budget availability}

The impact of budget availability over a 5-year programming period was determined. The budget varies as \$12M, \$13M, \$15M, and \$18M to investigate the change in optimal highway programs generated from the developed model, as shown in Figure 516. It shows that transportation agencies’ budget availability has an impact on the analysis result. A higher level of available budget leads to a higher net public benefits but lower $\mathrm{CO}_{2}$ emissions. The variation of available budget can lead the change in net public benefits, ranging from $-1.8 \%$ to $0.38 \%$, and $\mathrm{CO} 2$ emissions ranging from $-0.13 \%$ to $0.4 \%$.

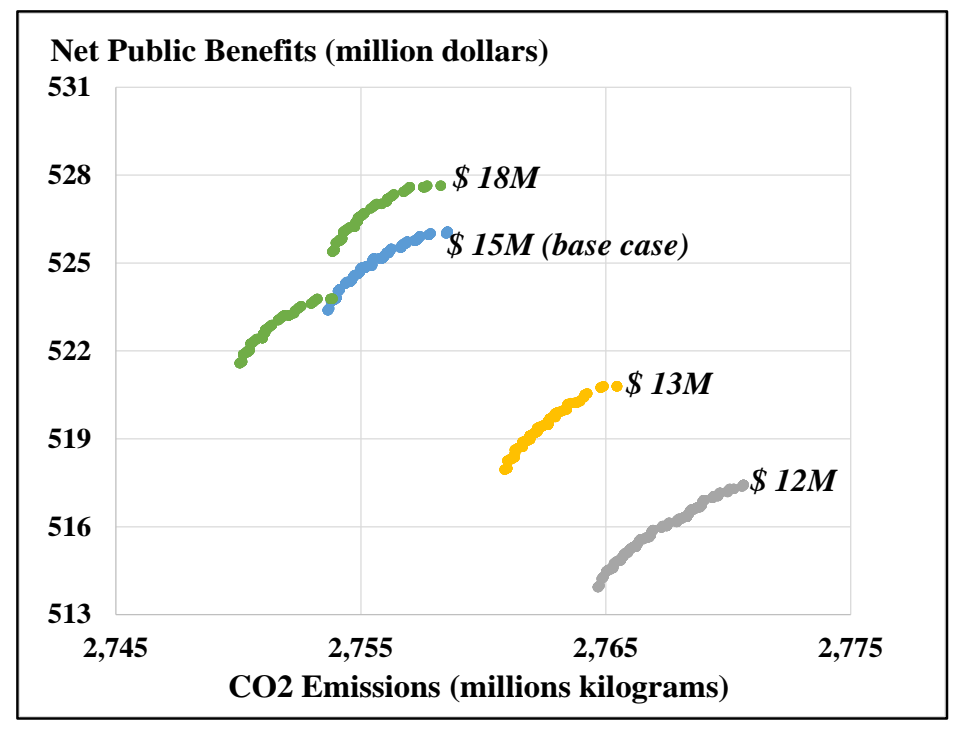

Figure 5-16 Sensitivity analysis from an uncertainty in budget availability 
5.8.3 Effect of the assumptions used in the vehicle fuel consumption estimating module

The uncertainties of some parameters considered during the development of the vehicle fuel consumption estimating module were investigated to check the impacts of the assumptions applied on those parameters to the final results. Accordingly, the analysis was performed on four parameters: (1) Benkelman Beam rebound deflection; (2) vehicle acceleration; (3) mean profile depth; and (4) gradient.

First, it shows a significant impact of Benkelman Beam rebound deflection on the generated set of optimal solutions. A pavement with a high value of deflection tends to generate a higher CO2 emission, as shown in Figure 5-17. However, it does not claim a relationship between the deflection and net public benefits from this result since all sets of solutions provide lower net public benefits, ranging from $-0.16 \%$ to $-2.97 \%$, when compared to the base case. In terms of the environmental impact, the uncertainty can cause $-2.45 \%$ to $2.82 \%$ variability in $\mathrm{CO}_{2}$ emissions.

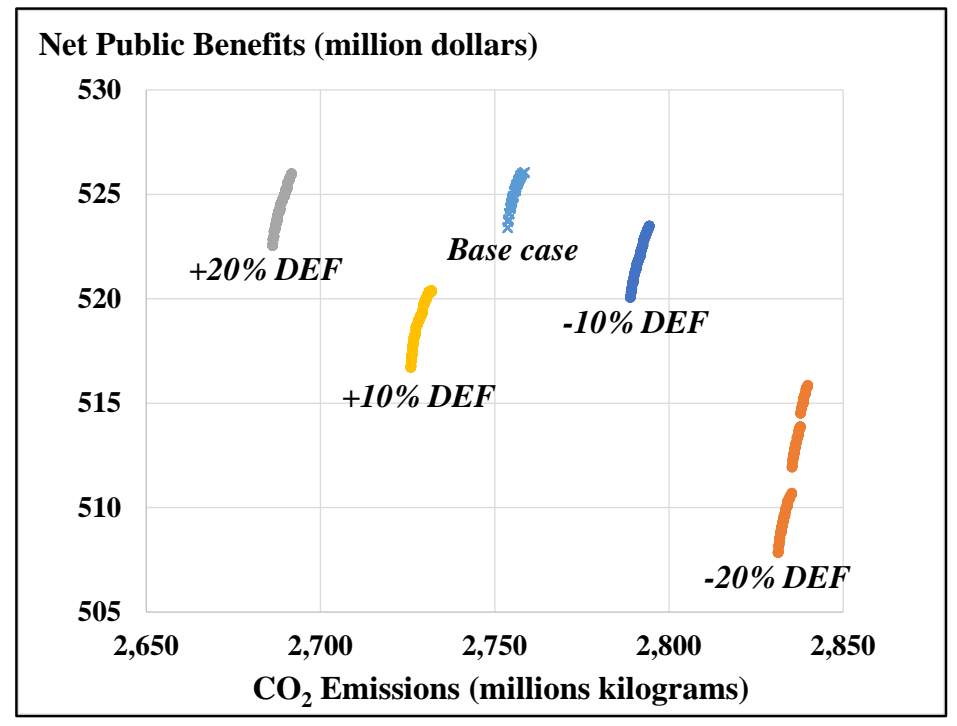

Figure 5-17 Sensitivity analysis from an uncertainty in Benkelman Beam rebound deflection 
Second, there is a variability in highway optimal solutions as a result of the uncertainty in vehicle acceleration. It shows a high impact in terms of $\mathrm{CO}_{2}$ emissions at a high vehicle acceleration rate, which is at $+10 \%$ and $+20 \%$ in Figure $5-18$. The change in CO2 emissions ranges from $-0.28 \%$ to $1.72 \%$ in this analysis. However, the relationship for net public benefits is not able to be concluded here, as no trend is significantly presented.

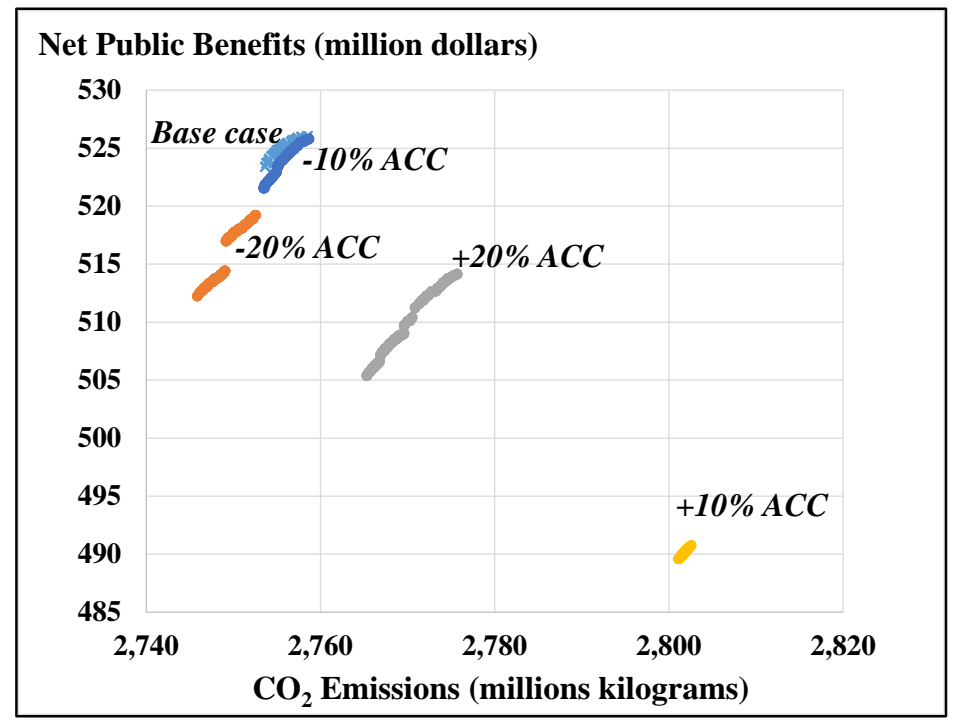

Figure 5-18 Sensitivity analysis from an uncertainty in vehicle acceleration

Third, Figure 5-19 illustrates a high variability among the sets of highway optimal programs at worse pavement conditions (at 10\% and 20\% mean profile depth). It shows a significant relationship between mean profile depth and $\mathrm{CO}_{2}$ emissions but not net public benefits. A worse pavement condition tends to lower $\mathrm{CO}_{2}$ emissions throughout the transportation network, while no relationship is shown for net public benefits. Numerically, based on this study, there are the variations between $-0.16 \%$ to $0.42 \%$ and $-0.1 \%$ to $-1.69 \%$ in $\mathrm{CO}_{2}$ emissions and net public benefits, respectively, while ranging mean depth profile from $-20 \%$ to $+20 \%$. 


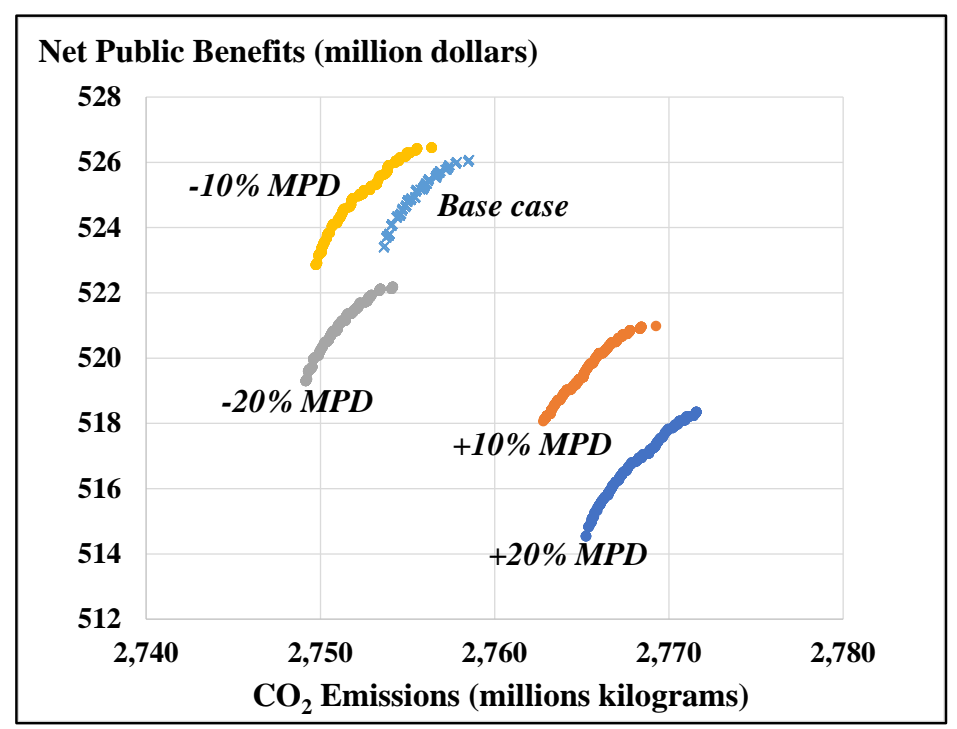

Figure 5-19 Sensitivity analysis from an uncertainty in mean profile depth

Fourth, Figure 5-20 demonstrated a significant impact of gradient on highway optimal programs by presenting a higher $\mathrm{CO}_{2}$ emission when a gradient of a road in the network increases. It shows the change in $\mathrm{CO}_{2}$ emissions from $-5.7 \%$ to $5.9 \%$ and net public benefits from $-1.63 \%$ to $1.33 \%$, when ranging the road gradient from $-20 \%$ to $20 \%$.

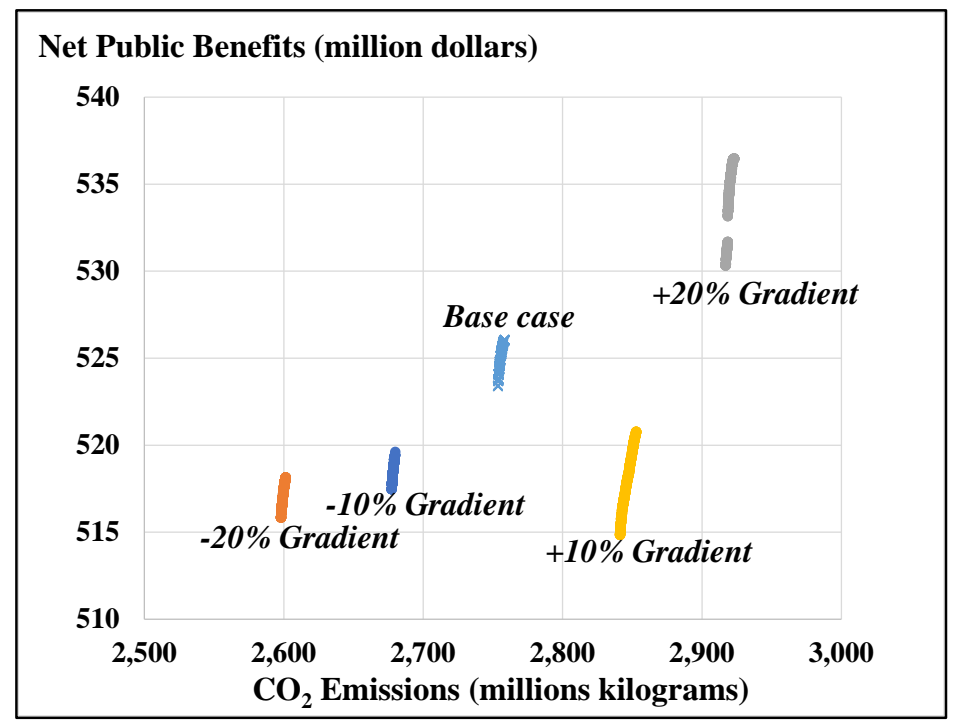

Figure 5-20 Sensitivity analysis from an uncertainty in gradient 


\subsection{Chapter Summary}

A new paradigm in highway rehabilitation efforts is required to promote the national environmental sustainability goal in current performance-based practice. To achieve this goal, a highway rehabilitation programming and optimization model was developed in this study in order to reduce $\mathrm{CO}_{2}$ emissions and maximize net public benefits in transportation networks resulting from implementing the rehabilitation treatment. The model includes six newly developed modules that present a novel scheme in highway rehabilitation programming and optimization. First, the treatment alternative identification module is designed to identify candidate rehabilitation treatment alternatives based on the variety in surface conditions of deteriorating pavement. Second, the pavement performance evaluating module provides the capability of forecasting the long-term pavement conditions over time along the predetermined analysis timespan. Third, the $\mathrm{CO}_{2}$ emission estimating module is developed to aid decision makers in evaluating the impact of rehabilitation decisions on $\mathrm{CO}_{2}$ emissions throughout transportation networks. Fourth, the travel-delay cost estimating module supports an estimation of the increased user cost due to travel delay during construction operations. Fifth, the road user cost savings estimating module provides the capability of estimating the expected savings in road user costs as a result from the improvement of pavement conditions after the treatment. Sixth, the multiobjective optimization module is designed to help decision makers and planners in optimizing highway rehabilitation programs that simultaneously minimize $\mathrm{CO}_{2}$ emissions and maximize net public benefits under limited funding.

The developed model is applied to the case study of the real transportation network in District 2, Florida, to evaluate the performance and capabilities of the model. The result 
presents its capabilities in generating optimal tradeoffs between $\mathrm{CO}_{2}$ emissions and net public benefits of highway rehabilitation efforts. To this end, this newly developed highway programming and optimization model should prove useful to state departments of transportation in generating cost-effective and environmentally-friendly highway rehabilitation programs that can properly serve the decision maker's preferences and requirements. 


\section{CHAPTER 6}

CONCLUSIONS

\subsection{Conclusions}

This dissertation aims to serve the national goals by improving current practices in highway transportation with an integration of environmental sustainability into programming and planning efforts. Transportation generates a high level of greenhouse gases (GHG) emissions, which primarily results from carbon dioxide $\left(\mathrm{CO}_{2}\right)$ emitted from the fossil fuel combustion in motor vehicles. As such, this research study was designed to focus on a reduction in total energy consumption and $\mathrm{CO}_{2}$ emissions of highway transportation networks. Three main parts of development were presented to achieve the research objectives, including: (1) measuring vehicle fuel consumption and public benefits in transportation networks; (2) evaluating the economic and environmental impacts of highway rehabilitation efforts on transportation networks; (3) developing a multi-objective optimization model to support decision making in programing and planning efforts of transportation networks.

The first part of this dissertation was designed to develop mathematical models for estimating vehicle fuel consumption and public benefits in such a way that facilitates an analysis at the network-level. This challenging task was performed with the statistical approach to provide an effective method for an estimation that is able to consider all relevant vehicle-road interaction factors using data available in the transportation database system. As a result, three main components related to energy consumption and vehicle operating costs are considered, including the vehicle fuel consumption rate, tire 
depreciation cost, and repair and maintenance cost rate. The relationships between these three components and the vehicle-road interaction factors were established. The mathematical models were then statistically developed and finally validated with the fieldinvestigation data.

The second part of this study was expanded to develop models for evaluating the impact of decision making in highway rehabilitation efforts on transportation networks in terms of economic and environmental platforms. This part introduces the main factors of highway rehabilitation programs that can affect the entire network's impact and how the mathematical models from the previous part can be applied in evaluating the impact. To this end, the economic and environmental impacts of highway rehabilitation efforts are evaluated with the development of the new model that is capable of: (1) identifying candidate rehabilitation treatment alternatives to deteriorating pavement; (2) evaluating and forecasting the impact of rehabilitation treatments on pavement performance; (3) estimating total energy consumption throughout the entire network as a result of highway rehabilitation decisions; (4) estimating the public costs as a result of the vehicle traveldelay from the speed reduction during construction operations, and (5) evaluating the impact of rehabilitation efforts on public benefits as the expected savings in road user costs after receiving rehabilitation. This model should prove useful to transportation agencies in selecting potential rehabilitation treatments that contribute to enhancing an achievement in economic and sustainability goals.

The third part of this dissertation was introduced with the development of a multiobjective optimization model to search for and identify highway rehabilitation programs that are capable of minimizing environmental impact in terms of $\mathrm{CO}_{2}$ emissions while 
maximizing public benefits under budget constraints. The developed model consists of six main modules that provide new capabilities in supporting decision making in highway rehabilitation efforts and promoting the sustainability concept in current highway practice. The developed model mentioned in the previous paragraph was improved and incorporated in the GA-based multi-objective optimization module to identify highway programs that satisfy the planning optimization objectives by taking into consideration three main decision variables, which include road selection, treatment timing, and treatment method. The application example was applied to evaluate the performance and capabilities of the model in generating optimal tradeoffs between $\mathrm{CO} 2$ emissions and net public benefits of highway rehabilitation efforts. This new-developed model enables planners and decision makers in implementing highway programs that serve both cost-effective and environmental sustainability goals.

\subsection{Research Contributions}

The contributions of this research can be concluded as follows:

1. The identification of the main vehicle-pavement interaction factors and their impacts to facilitate estimating total fuel consumption of transportation networks.

2. The assessment of the impact of decision making in highway rehabilitation programs on total energy consumption and public benefits/costs of transportation networks.

3. The integration of the environmental sustainability in highway rehabilitation planning and optimization efforts that enable the sustainability goal in highway rehabilitation decision making in reducing the environmental impact in transportation networks. 


\subsection{Limitations and Recommendation for Future Research}

Based on the research development in this study, some extensions can be further performed to improve the capabilities of the developed models and their applications. The recommendations for future research are provided corresponding with each part of research as follows.

Chapter 3: Measuring vehicle fuel consumption and public benefits in transportation $\underline{\text { networks }}$

The mathematical models were developed in this chapter to support an estimation of vehicle fuel consumption rate, tire depreciation rate, and repair and maintenance cost rate at the network level. However, the limited set of field data was used for the model validation in this study. Therefore, increasing the number of field investigation data that can be used for validation will improve the efficiency and accuracy of the models.

Chapter 4: Evaluating the economic and environmental impacts of rehabilitation efforts on $\underline{\text { transportation networks }}$

The development of the model in this chapter can be further extended to advance its capabilities as follows.

(1) The user equilibrium in transportation networks may be adopted in the travel-delay cost estimating module in order to improve applications of the model.

(2) The scope of the road user cost saving estimating module can be expanded to incorporate other types of social benefits, such as safety improvement, traffic congestion reduction, and travel time savings. 


\section{Chapter 5: Optimizing highway rehabilitation projects}

Some further improvements can be performed to improve the capabilities of the multi-objective optimization model developed in this chapter, as follows.

(1) The uncertainty of highway decisions and stochastic nature of highway transportation networks can be considered, such as the uncertainty of future pavement conditions, availability of transportation agencies' budget, a variation of fuel price, and traffic growth rate. This can contribute to the perception of decision makers in selecting more effective highway rehabilitation programs under the constraints and uncertainty environment.

To this end, the scope of uncertainty can be expanded to include more of those aforementioned factors in the sensitivity analysis. Moreover, some approaches can be performed to incorporate the risk and uncertainty factors in the analysis. For example, Monte Carlo Simulation can be applied to furnish planners and decision makers with a range of possible outcomes that allows for better decisions under uncertainty.

(2) The developed model may incorporate other planning optimization objectives, such as construction cost, in the analysis (if applicable). This can introduce new dimensions and perceptions to transportation planners in effectively selecting rehabilitation programs.

(3) The developed model may include more realistic and practical options for optimizing highway rehabilitation efforts by increasing opportunities for decision makers to repair a road several times over a programming cycle. 
(4) The concept of the environmentally-conscious decision-support model developed in this study may be applied to the rigid pavement structure or the combination of flexible and rigid pavements in transportation networks. 


\section{LIST OF REFERENCES}

AASHTO (2010). User and Non-User Benefit Analysis for Highways, American Association of State Highway and Transportation Officials, Washington D.C.

Abaza, K. A. (2002). "Optimum flexible pavement life-cycle analysis model." Journal of Transportation Engineering, 128(6), 542-549.

AI\&T (2006). "Guidelines for Assessing Pavement Preservation Treatments and Strategies." Alberta Infrastructure \& Transportation, Edmonton, Alberta, Canada.

Akbarian, M., Moeini-Ardakani, S., Ulm, F.-J., and Nazzal, M. (2012). "Mechanistic approach to pavement-vehicle interaction and its impact on life-cycle assessment." Transportation Research Record: Journal of the Transportation Research Board(2306), 171-179.

Akcelik, R., and Besley, M. (2003). "Operating cost, fuel consumption, and emission models in aaSIDRA and aaMOTION." Proc., 25th Conference of Australian Institutes of Transport Research (CAITR 2003), 1-15.

Amos, D. (2006). "Pavement smoothness and fuel efficiency: an analysis of the economic dimensions of the Missouri Smooth Road Initiative."

ASCE (2013). "2013 Report card for America's infrastructure ", American Society of Civil Engineers.

Barnes, G., and Langworthy, P. (2004). "Per mile costs of operating automobiles and trucks." Transportation Research Record: Journal of the Transportation Research Board(1864), 71-77.

Bennett, C., and Greenwood, I. (2003). "Volume 7: Modeling road user and environmental effects in HDM-4, Version 3.0, international study of highway development and management tools (ISOHDM), World Road Association (PIARC)." ISBN.

Brooks, R. M. (2012). "Acceleration Characteristics of Vehicle in Rural Pennsylvania." ANALYSIS, 1(5), 5.22.

Caltrans (2015). "CA4PRS Implementation Project for Rapid Rehabilitation." California Department of Transportation.

Carnahan, J., Davis, W., Shahin, M., Keane, P., and Wu, M. (1987). "Optimal maintenance decisions for pavement management." Journal of Transportation Engineering, 113(5), 554-572.

Cass, D., and Mukherjee, A. (2011). "Calculation of greenhouse gas emissions for highway construction operations by using a hybrid life-cycle assessment approach: case 
study for pavement operations." Journal of Construction Engineering and Management, 137(11), 1015-1025.

Chan, W., Fwa, T., and Tan, C. (1994). "Road-maintenance planning using genetic algorithms. I: Formulation." Journal of Transportation Engineering, 120(5), 693709.

Chan, W. T., Fwa, T., and Tan, J. (2003). "Optimal fund-allocation analysis for multidistrict highway agencies." Journal of Infrastructure Systems, 9(4), 167-175.

Chatti, K., and Zaabar, I. (2012). Estimating the effects of pavement condition on vehicle operating costs, Transportation Research Board.

Chootinan, P., Chen, A., Horrocks, M. R., and Bolling, D. (2006). "A multi-year pavement maintenance program using a stochastic simulation-based genetic algorithm approach." Transportation Research Part A: Policy and Practice, 40(9), 725-743.

Copeland, L. (1998). User's Manual for QUEWZ-98, Texas Transportation Institute, the Texas A \& M University System.

de la Garza, J. M., Akyildiz, S., Bish, D. R., and Krueger, D. A. (2011). "Network-level optimization of pavement maintenance renewal strategies." Advanced Engineering Informatics, 25(4), 699-712.

de la Garza, J. M., and Krueger, D. A. (2007). "Simulation of highway renewal asset management strategies."

Deb, K., Pratap, A., Agarwal, S., and Meyarivan, T. (2002). "A fast and elitist multiobjective genetic algorithm: NSGA-II." Evolutionary Computation, IEEE Transactions on, 6(2), 182-197.

Dewan, S., and Smith, R. (2002). "Estimating International Roughness Index from pavement distresses to calculate vehicle operating costs for the San Francisco Bay area." Transportation Research Record: Journal of the Transportation Research Board(1816), 65-72.

Dhakal, K. P., and Oh, J. S. (2011). "Integrating Sustainability into Highway Projects: Sustainability Indicators and Assessment Tool for Michigan Roads." Proc., Reston, VA: ASCE copyright Proceedings of the First Transportation and Development Institute Congress; March 13-16, 2011, Chicago, Illinois| d 20110000, American Society of Civil Engineers.

EPA (2005). "Emission Facts - Average carbon dioxide emissions resulting from gasoline and diesel fuel." US EPA, February, United States Environmental Protection Agency.

EPA (2013a). "Overview of Greenhouse Gases." 
EPA (2013b). "Sources of Greenhouse Gas Emissions."

Epps, J., Leahy, R., Mitchell, T., Ashmore, C., Seeds, S., Alavi, S., and Monismith, C. (1999). "Westrack-The road to performance-related specifications." Proc., Proceedings of International Conference on Accelerated Pavement Testing. Reno, NV.

Ferreira, A., Antunes, A., and Picado-Santos, L. 1. s. (2002). "Probabilistic segment-linked pavement management optimization model." Journal of Transportation Engineering, 128(6), 568-577.

Ferreira, A., Picado-Santos, L. d., Wu, Z., and Flintsch, G. (2011). "Selection of pavement performance models for use in the Portuguese PMS." International Journal of Pavement Engineering, 12(1), 87-97.

FHWA (2012). "Moving Ahead for Progress in the 21st Century Act (MAP-21) A Summary of Highway Provisions." Federal Highway Administration.

FHWA (2014). "Annual vehicle distance traveled in miles and related data - 2012(1) by highway category and vehicle type.".

Fwa, T., Chan, W., and Hoque, K. (2000). "Multiobjective optimization for pavement maintenance programming." Journal of Transportation Engineering, 126(5), 367374.

Fwa, T., and Farhan, J. (2012). "Optimal multiasset maintenance budget allocation in Highway asset management." Journal of Transportation Engineering, 138(10), 1179-1187.

Fwa, T. F., and Sinha, K. C. (1991). "Pavement performance and life-cycle cost analysis." Journal of Transportation Engineering, 117(1), 33-46.

Geoffroy, D. N. (1996). Cost-effective preventive pavement maintenance.

Gillespie, T. D. (1992). "Everything you always wanted to know about the iri, but were afraid to ask." Proc., Road Profile Users Group Meeting, Lincoln, Nebraska.

Giustozzi, F., Crispino, M., and Flintsch, G. (2012). "Multi-attribute life cycle assessment of preventive maintenance treatments on road pavements for achieving environmental sustainability." The International Journal of Life Cycle Assessment, 17(4), 409-419.

Hall, K. T., Correa, C. E., Carpenter, S. H., and Elliott, R. (2001). Rehabilitation strategies for highway pavements, Transportation Research Board. 
Hicks, R., Moulthrop, J., and Daleiden, J. (1999). "Selecting a preventive maintenance treatment for flexible pavements." Transportation Research Record: Journal of the Transportation Research Board(1680), 1-12.

Higgins, J. (2005). "The radical statistician." Duxbury Advanced series, Duxbury, Massachusetts, United States.

Hong, F., and Prozzi, J. A. (2013). "Pavement Deterioration Model Incorporating Unobserved Heterogeneity for Optimal Life-Cycle Rehabilitation Policy." Journal of Infrastructure Systems.

INDOT (2001). "Pavement Surface Report." Pavement Management Unit, Indiana Department of Transportation, Indianapolis, Indiana.

Irfan, M. (2010). "A framework for developing optimal pavement life-cycle activity profiles."

Irfan, M., Khurshid, M. B., Bai, Q., Labi, S., and Morin, T. L. (2012). "Establishing optimal project-level strategies for pavement maintenance and rehabilitation-A framework and case study." Engineering Optimization, 44(5), 565-589.

Irfan, M., Khurshid, M. B., Labi, S., and Flora, W. (2009). "Evaluating the cost effectiveness of flexible rehabilitation treatments using different performance criteria." Journal of Transportation Engineering, 135(10), 753-763.

Irfan, M., Khurshid, M. B., Labi, S., and Sinha, K. C. (2008). "Cost-Effectiveness of Rehabilitation Alternatives-The Case for Flexible Pavements." Proc., Seventh International Conference on Managing Pavement Assets.

Kahn Ribeiro, S., Kobayashi, S., Beuthe, M., Gasca, J., Greene, D., Lee, D. S., Muromachi, Y., Newton, P. J., Plotkin, S., and Sperling, D. (2007). "Transport and its infrastructure." Climate change, 323-385.

Khurshid, M. B. (2010). "A framework for establishing optimal performance thresholds for highway asset interventions." PURDUE UNIVERSITY.

Klaubert, E. C. (2001). "Highway effects on vehicle performance."

Labi, S., and Sinha, K. C. (2003). "The effectiveness of maintenance and its impact on capital expenditures."

Lamptey, G., Ahmad, M. Z., Labi, S., and Sinha, K. C. (2005). "Life cycle cost analysis for INDOT pavement design procedures."

Lamptey, G., Labi, S., and Li, Z. (2008). "Decision support for optimal scheduling of highway pavement preventive maintenance within resurfacing cycle." Decision Support Systems, 46(1), 376-387. 
Lidicker, J., Sathaye, N., Madanat, S., and Horvath, A. (2012). "Pavement resurfacing policy for minimization of life-cycle costs and greenhouse gas emissions." Journal of Infrastructure Systems, 19(2), 129-137.

Limsawasd, C., Orabi, W., and Athigakunagorn, N. (2016). "Optimizing Highway Rehabilitation Decisions to Minimize Fuel Consumption in Transportation Networks."

Liu, F., and Wang, K. (1996). "Pavement performance-oriented network optimization system." Transportation Research Record: Journal of the Transportation Research Board(1524), 86-93.

Mathew, B. S., and Isaac, K. P. (2014). "Optimisation of maintenance strategy for rural road network using genetic algorithm." International Journal of Pavement Engineering, 15(4), 352-360.

Mbwana, J., and Turnquist, M. (1996). "Optimization modeling for enhanced networklevel pavement management system." Transportation Research Record: Journal of the Transportation Research Board(1524), 76-85.

Mn/DOT (2001). "Network Level Bituminous Decision Tree." Minnesota Department of Transportation, Office of Materials and Road Research, St. Paul, MN.

ODOT (1999). Pavement Design and Rehabilitation Manual, Ohio Department of Transportation.

OECD (2005). "Economic Evaluation of Long-Life Pavements Phase 1." Organisation for Economic Co-Operation and Development, Paris, France.

Orabi, W., and El-Rayes, K. (2011). "Optimizing the rehabilitation efforts of aging transportation networks." Journal of Construction Engineering and Management, 138(4), 529-539.

Paterson, W., and Attoh-Okine, B. (1992). "Simplified models of paved road deterioration based on HDM-III." Proc., Transportation Research Board, 71st Annual Meeting: Washington, DC, USA.

Paterson, W. D., and Watanatada, T. (1985). "Relationships between vehicle speed, ride quality, and road roughness." Measuring Road Roughness and Its Effects on User Cost and Comfort. ASTM Special Technical Publication (STP), 884, 89-110.

Sathaye, N., and Madanat, S. (2011). "A bottom-up solution for the multi-facility optimal pavement resurfacing problem." Transportation Research Part B: Methodological, 45(7), 1004-1017. 
Sathaye, N., and Madanat, S. (2012). "A bottom-up optimal pavement resurfacing solution approach for large-scale networks." Transportation Research Part B: Methodological, 46(4), 520-528.

Sayers, M. W., Gillespie, T. D., and Paterson, W. D. (1986). Guidelines for conducting and calibrating road roughness measurements.

Sayers, M. W., Gillespie, T. D., and Queiroz, A. (1986). "The international road roughness experiment. Establishing correlation and a calibration standard for measurements."

Sayers, M. W., and Karamihas, S. M. (1998). "The little book of profiling." the Regent of the University of Michigan, 2.

Shafizadeh, K. R., Mannering, F. L., and Pierce, L. M. (2002). "A statistical analysis of factors associated with driver-perceived road roughness on urban highways." Citeseer.

Sharaf, E., and Mandeel, F. (1998). "An analysis of the impact of different priority setting techniques on network pavement condition." Proc., Fourth international conference on managing pavements.

Song, L. (2011). "NGPM: A NSGA-II program in MATLAB, version 1.4, file exchange. MathWorks, 20 October 2011."

Statistics, U. S. B. o. L. (2014). "CPI Detailed Report Data for December 2014."

Sumitsawan, P., Ardekani, S. A., and Romanoschi, S. (2009). "Effect of pavement type on fuel consumption and emissions." Proc., Proceedings of the 2009 Mid-Continent transportation research symposium. Ames, Iowa: Iowa State University.

Taylor, G., Farrel, P., and Woodside, A. (2002). "Additional Analysis of the Effect of Pavement Structures on Truck Fuel Consumption." National Research Council of Canada (NRC), Ottawa, Ontario.

Taylor, G., and Patten, J. (2006). "Effects of pavement structure on vehicle fuel consumption-Phase III."

Tucson (2012). "Asphalt Pavement Treatments (https://www.tucsonaz.gov/files/gov/Asphalt_Pavement_Treatments.pdf)."

Wade, M., DeSombre, R., and Peshkin, D. (2001). "High volume/high speed asphalt roadway preventive maintenance surface treatments."

Wang, F., Zhang, Z., and Machemehl, R. (2003). "Decision-making problem for managing pavement maintenance and rehabilitation projects." Transportation Research Record: Journal of the Transportation Research Board(1853), 21-28. 
Wang, K., and Liu, F. (1997). "Fuzzy set-based and performance-oriented pavement network optimization system." Journal of infrastructure systems, 3(4), 154-159.

Wang, T. (2013). Reducing greenhouse gas emissions and energy consumption using pavement maintenance and rehabilitation: Refinement and application of a life cycle assessment approach, University of California, Davis.

Wang, T., Lee, I.-S., Kendall, A., Harvey, J., Lee, E.-B., and Kim, C. (2012). "Life cycle energy consumption and GHG emission from pavement rehabilitation with different rolling resistance." Journal of Cleaner Production, 33, 86-96.

Watanatada, T., Dhareshwar, A. M., and Rezende Lima, P. (1987). "Vehicle speeds and operating costs." Models for Road Planning and Management, The World Bank.

Wilde, W. J., Waalkes, S., and Harrison, R. (1999). Life cycle cost analysis of Portland cement concrete pavements, Citeseer.

Wu, Z., Groeger, J. L., Simpson, A. L., and Hicks, R. G. (2010). "Performance evaluation of various rehabilitation and preservation treatments."

Yu, B., and Lu, Q. (2012). "Life cycle assessment of pavement: Methodology and case study." Transportation Research Part D: Transport and Environment, 17(5), 380388.

Zaabar, I., and Chatti, K. (2010). "Calibration of HDM-4 models for estimating the effect of pavement roughness on fuel consumption for US conditions." Transportation Research Record: Journal of the Transportation Research Board(2155), 105-116.

Zaabar, I., and Chatti, K. (2011). "A field investigation of the effect of pavement type on fuel consumption." Proc., 1st Congress of the Transportation and Development Institute of ASCE.

Zaniewski, J. P. (1989). "Effect of pavement surface type on fuel consumption." Portland Cement Association.

Zaniewski, J. P., Butler, B., Cunningham, G., Elkins, G., and Paggi, M. (1982). "Vehicle operating costs, fuel consumption, and pavement type and condition factors. Final report Sep 79-Oct 81." Texas Research and Development Foundation, Austin (USA).

Zhang, H., Keoleian, G., and Lepech, M. (2008). "An integrated life cycle assessment and life cycle analysis model for pavement overlay systems." Proc., 1st International symposium on life-cycle civil engineering, Varenna, Italy, Citeseer, 907-915.

Zhang, H., Keoleian, G. A., and Lepech, M. D. (2012). "Network-level pavement asset management system integrated with life-cycle analysis and life-cycle optimization." Journal of Infrastructure Systems. 
Zhang, H., Keoleian, G. A., Lepech, M. D., and Kendall, A. (2010). "Life-cycle optimization of pavement overlay systems." Journal of infrastructure systems.

Zhang, H., Lepech, M. D., Keoleian, G. A., Qian, S., and Li, V. C. (2009). "Dynamic lifecycle modeling of pavement overlay systems: Capturing the impacts of users, construction, and roadway deterioration." Journal of Infrastructure Systems, 16(4), 299-309.

Zimmerman, K., and Peshkin, D. (2004). "Issues in integrating pavement management and preventive maintenance." Transportation Research Record: Journal of the Transportation Research Board(1889), 13-20.

Zimmerman, K. A., Grogg, M. G., and Bozkurt, D. (2002). "Effects of maintenance treatments on asphalt concrete pavement management." 


\title{
APPENDIX
}

\section{NOTATION}

\author{
$\mathrm{D}_{\mathrm{r}} \quad=\quad$ Construction duration that affects road section ( $\left.\mathrm{r}\right)$; \\ $\mathrm{DRI}_{\mathrm{r}}^{\mathrm{y}}=\quad$ Rate of tire depreciation savings of road section (r) during year $(\mathrm{y})$ without \\ treatment; \\ $\mathrm{DRN}_{\mathrm{r}}^{\mathrm{y}}=\quad$ Rate of tire depreciation savings of road section (r) during year (y) with \\ treatment; \\ DS $^{\mathrm{y}}=$ Total tire depreciation cost savings during year (y) of the highway \\ rehabilitation program; \\ $\mathrm{EF}_{\mathrm{i}}=$ Emission factor when fuel type (i) is used in vehicle; \\ $\mathrm{FS}^{\mathrm{y}}=$ Total fuel consumption cost savings during year $(\mathrm{y})$ of the highway \\ rehabilitation program; \\ $\mathrm{FR}_{\mathrm{W}, \mathrm{r}}=\quad$ Fuel consumption rate on road section (r) under work-zone conditions; \\ $\mathrm{FRI}_{\mathrm{r}}^{\mathrm{y}}=\quad$ Fuel consumption rate on road section ( $\mathrm{r}$ ) during year $(\mathrm{y})$ without \\ treatment; \\ $\mathrm{FRN}_{\mathrm{r}}^{\mathrm{y}}=\quad$ Fuel consumption rate on road section ( $\mathrm{r}$ ) at year (y) of the highway \\ rehabilitation program; \\ ir $=$ Discount rate used for the cost-benefit analysis; \\ $\mathrm{IRI}_{\mathrm{pre}}^{\mathrm{r}}=\quad$ IRI value on road section (r) before an application of treatment option; \\ $\mathrm{IRI}_{\text {post }}^{\mathrm{r}}=\quad$ IRI value on road section ( $\mathrm{r}$ ) after an application of treatment option; \\ $\mathrm{L}_{\mathrm{r}} \quad=\quad$ Length of road section (r); \\ $\mathrm{MRI}_{\mathrm{r}}^{\mathrm{y}}=\quad$ Rate of repair and maintenance cost savings of road section (r) during year \\ (y) without rehabilitation; \\ $\mathrm{MRN}_{\mathrm{r}}^{\mathrm{y}}=\quad$ Rate of repair and maintenance cost savings of road section (r) during year \\ (y) with rehabilitation;
}




$$
\begin{aligned}
& \mathrm{MS}^{\mathrm{y}}=\text { Total repair and maintenance cost savings during year }(\mathrm{y}) \text { of the highway } \\
& \text { rehabilitation program; } \\
& \mathrm{PJ}_{\mathrm{S}}^{\mathrm{r}} \quad=\quad \text { Performance jump at the time of application for treatment option; } \\
& \mathrm{r} \quad=\quad \text { Road section }(\mathrm{r}=1 \text { to } \mathrm{R}) \text {; } \\
& \mathrm{R} \quad=\quad \text { Number of road sections in the network; } \\
& \mathrm{S}_{\mathrm{F}, \mathrm{r}}=\quad \text { Average vehicle speed on road section ( } \mathrm{r} \text { ) under free-flow conditions; } \\
& \mathrm{S}_{\mathrm{W}, \mathrm{r}}=\quad \text { Average vehicle speed on road section (r) under work-zone conditions; } \\
& \mathrm{T}_{\mathrm{F}, \mathrm{r}}=\quad \text { Travel time on road section ( } \mathrm{r} \text { ) under free-flow conditions; } \\
& \mathrm{T}_{\mathrm{W}, \mathrm{r}}=\quad \text { Travel time on road section ( } \mathrm{r} \text { ) during construction operations; } \\
& \text { TCC }=\text { Total } \mathrm{CO}_{2} \text { emission due to construction operations; } \\
& \text { TFO }=\text { Total } \mathrm{CO}_{2} \text { emission during the regular operation; } \\
& \mathrm{TPB}=\text { Total expected public benefits of the highway rehabilitation program; } \\
& \text { TTC }=\quad \text { Total cost of travel delay during construction; } \\
& \text { UT = Unit time value (dollars per hour); } \\
& \mathrm{V}_{\mathrm{r}} \quad=\quad \text { Traffic volume on road section }(\mathrm{r}) \text {; } \\
& \mathrm{V}_{\mathrm{F}, \mathrm{r}}=\quad \text { Traffic volume on road section ( } \mathrm{r} \text { ) under free-flow conditions; } \\
& \mathrm{V}_{\mathrm{W}, \mathrm{r}}=\quad \text { Traffic volume on road section (r) during construction operations; } \\
& \mathrm{y} \quad=\quad \text { Analysis year after rehabilitation }(\mathrm{y}=1 \text { to } \mathrm{Y}) \text {; } \\
& \mathrm{Y} \quad=\quad \text { Number of years to new rehabilitation effort; } \\
& \Delta \mathrm{DR}_{\mathrm{r}}^{\mathrm{y}}=\quad \text { Rate of tire depreciation savings of road section (r) at year (y) after } \\
& \text { rehabilitation; } \\
& \Delta \mathrm{FR}_{\mathrm{r}}^{\mathrm{y}}=\quad \text { Rate of fuel consumption savings of road section (r) at year (y) after } \\
& \text { rehabilitation; }
\end{aligned}
$$


$\Delta \mathrm{MR}_{\mathrm{r}}^{\mathrm{y}}=\quad$ Rate of repair and maintenance savings of road section (r) at year (y) after rehabilitation;

$\Delta \mathrm{T}_{\mathrm{r}} \quad=\quad$ Change in travel time or travel delay due to rehabilitation; 
VITA

\section{CHARINEE LIMSAWASD}

Born, Singburi, Thailand

1997-2001

B.Eng., Civil Engineering

Chulalongkorn University

Bangkok, Thailand

2001-2005

Civil Engineer

Provincial Electricity Authority of Thailand

2005-2008

M.Eng., Civil Engineering

Chulalongkorn University

Bangkok, Thailand

2009-Present

Lecturer

Faculty of Engineering at Kamphaeng Saen

Kasetsart University, Thailand

2011-2016

Ph.D. Candidate

Department of Civil \& Environmental Engineering

Florida International University

Graduate Research Assistant

OHL School of Construction

Florida International University

\section{PUBLICATIONS AND PRESENTATIONS}

Kang, Y., Kim, C., Son, H., Lee, S. and Limsawasd, C. (2013). Comparison of preproject planning for green and conventional buildings. Journal of Construction Engineering and Management, 139 (11).

Limsawasd, C., Orabi, W., and Pumpichet, S. (2015). Estimating Fuel Consumption from Highway-Rehabilitation Program Implementation on Transportation Networks. Paper presented at ASCE Computing in Civil Engineering Workshop 2015, June 21-23, 2015, Austin, Texas.

Limsawasd, C. and Orabi, W. (2015). Optimizing Environmental Sustainability and Public benefits of Transportation Network Programs. Paper presented at 2015 International 
Construction Specialty Conference, Canadian Society for Civil Engineering, June 8-10, 2015, Vancouver, Canada.

Limsawasd, C., Orabi, W., and Athigakunagorn, N. (2016). Optimizing the Highway Rehabilitation Decisions to Enhance Environmental Sustainability in Transportation Networks. Construction Research Congress 2016, May 31- June 2, 2016, San Juan, Puerto Rico. (Paper accepted)

Limsawasd, C. and Orabi, W. (February, 2015). Maximizing Environmental Sustainability and Public Benefits of Highway Construction Programs. Poster presentation at the FIU CEE graduate Research day - Poster session, Florida International University, Miami, FL.

Limsawasd, C. and Orabi, W. (October 2014). Maximizing Environmental Sustainability and Public Benefits of Highway Construction Programs. Poster presentation at The 30th Anniversary Celebration, FIU College of Engineering and Computing, Miami, FL. 